NATIONAL LABORATORY

\title{
Supporting Documentation for the 2008 Update to the Insulation Fact Sheet
}

January 31, 2008

Prepared by

Therese K. Stovall

Senior Research Engineer

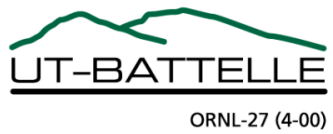




\title{
DOCUMENT AVAILABILITY
}

Reports produced after January 1, 1996, are generally available free via the U.S. Department of Energy (DOE) Information Bridge.

Web site http://www.osti.gov/bridge

Reports produced before January 1, 1996, may be purchased by members of the public from the following source.

\author{
National Technical Information Service \\ 5285 Port Royal Road \\ Springfield, VA 22161 \\ Telephone 703-605-6000 (1-800-553-6847) \\ TDD 703-487-4639 \\ Fax 703-605-6900 \\ E-mail info@ntis.gov \\ Web site http://www.ntis.gov/support/ordernowabout.htm
}

Reports are available to DOE employees, DOE contractors, Energy Technology Data Exchange (ETDE) representatives, and International Nuclear Information System (INIS) representatives from the following source.

Office of Scientific and Technical Information

P.O. Box 62

Oak Ridge, TN 37831

Telephone 865-576-8401

Fax 865-576-5728

E-mail reports@osti.gov

Web site http://www.osti.gov/contact.html

This report was prepared as an account of work sponsored by an agency of the United States Government. Neither the United States Government nor any agency thereof, nor any of their employees, makes any warranty, express or implied, or assumes any legal liability or responsibility for the accuracy, completeness, or usefulness of any information, apparatus, product, or process disclosed, or represents that its use would not infringe privately owned rights. Reference herein to any specific commercial product, process, or service by trade name, trademark, manufacturer, or otherwise, does not necessarily constitute or imply its endorsement, recommendation, or favoring by the United States Government or any agency thereof. The views and opinions of authors expressed herein do not necessarily state or reflect those of the United States Government or any agency thereof. 
Engineering Science and Technology Division

\title{
Supporting Documentation for the 2008 Update to the Insulation Fact Sheet
}

\author{
Therese K. Stovall
}

Date Published: January 2008

\author{
Prepared by \\ OAK RIDGE NATIONAL LABORATORY \\ Oak Ridge, Tennessee 37831-6283 \\ managed by \\ UT-BATTELLE, LLC \\ for the \\ U.S. DEPARTMENT OF ENERGY \\ under contract DE-AC05-00OR22725
}




\section{CONTENTS}

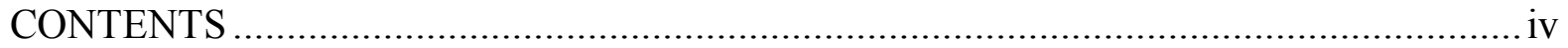

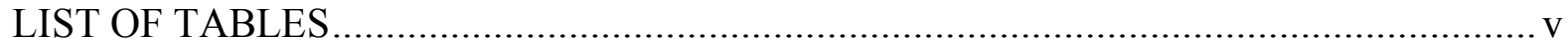

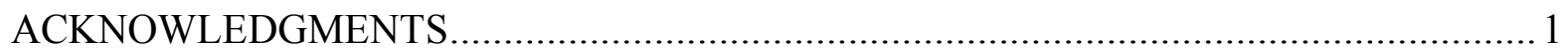

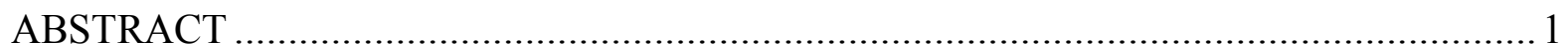

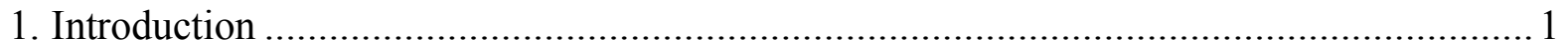

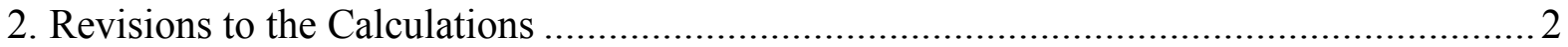

2.1 The ZipCode Insulation Program Methodology ............................................. 2

2.2 Performance Assumptions .............................................................................. 3

2.3 Cost Assumptions..................................................................................... 4

3. Summary of Revisions to the Insulation Fact Sheet .................................................. 6

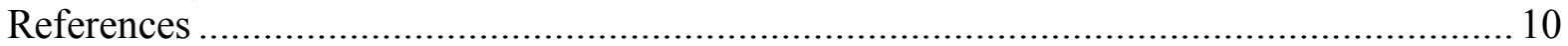

Appendix A Public Review Comments ......................................................................... 12

Appendix B Values Used in the 2008 Update to the Insulation Fact Sheet......................... 14

Appendix C Text of the 2008 DOE Insulation Fact Sheet.......................................... 44 


\section{LIST OF TABLES}

Table

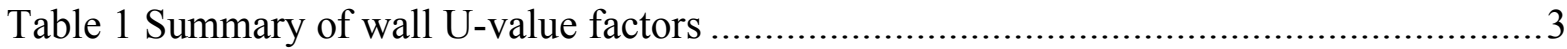

Table 2 Heating and cooling system performance assumption changes ............................ 4

Table 3 Comparison of selected installed costs for insulation measures in new houses ......... 8

Table 4 Retail material costs in 31 states reviewed in 2006 and 2007 .............................. 9

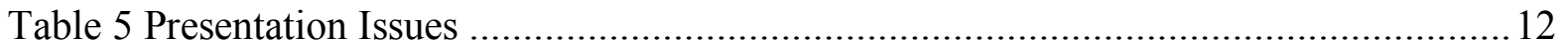

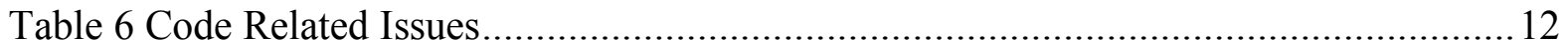

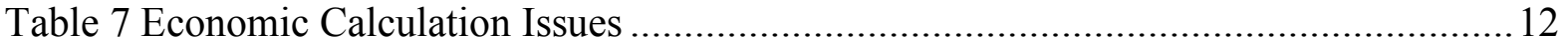

Table 8 Thermal Calculation Issues................................................................... 13

Table 9 Insulation cost and performance parameters used for 2008 fact sheet analysis for

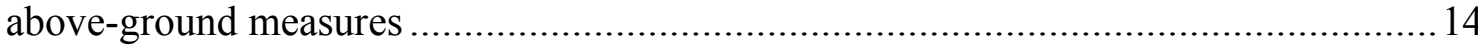

Table 10 Insulation cost used for 2008 fact sheet analysis for foundation measures ............ 16

Table 11 Residential Energy Prices for 2008 Update ...................................................... 17

Table 12 Projected fuel price escalation indices (excluding general inflation) for Census Region 1 (Connecticut, Maine, Massachusetts, New Hampshire, New Jersey, New York, Pennsylvania, Rhode Island, Vermont)....

Table 13 Projected fuel price escalation indices (excluding general inflation) for Census Region 2 (Illinois, Indiana, Iowa, Kansas, Michigan, Minnesota, Missouri, Nebraska, North Dakota, Ohio, South Dakota, Wisconsin).

Table 14 Projected fuel price escalation indices (excluding general inflation) for Census Region 3 (Alabama, Arkansas, Delaware, District of Columbia, Florida, Georgia, Kentucky, Louisiana, Maryland, Mississippi, North Carolina, Oklahoma, South Carolina, Tennessee.

Table 15 Projected fuel price escalation indices (excluding general inflation) for Census Region 4 (Alaska, Arizona, California, Colorado, Hawaii, Idaho, Montana, Nevada, New Mexico, Oregon, Utah, Washington, Wyoming)

Table 16 Zip codes, heating degree days (HDD, base $65, \div 1000$ ), cooling degree hours ( $\mathrm{CDH}$, base $74 \mathrm{~F}, \div 1000$ ), fuel price escalation region (FER), insulation cost factor (ICF), IECC region. 


\title{
ACKNOWLEDGMENTS
}

The Building Technology Program within the Office of Energy Efficiency and Renewable Energy of the U.S. Department of Energy sponsors the DOE Insulation Fact Sheet. This update also received support and funding from the North American Insulation Manufacturers' Association.

\begin{abstract}
The DOE Insulation Fact Sheet is designed to help homeowners make informed decisions about home insulation. The Fact Sheet was recently updated to accomplish multiple goals. First, the insulation cost data and energy prices used to calculate life-cycle costs for insulation applications were updated. Second, insulation materials and practices have progressed. Third, the evolution of the Fact Sheet from a paper pamphlet form to an interactive web site was in need of improvement. The information provided in this supporting documentation describes the basis for these changes.
\end{abstract}

\section{INTRODUCTION}

The DOE Insulation Fact Sheet was first published in 1978 with updates in 1982, 1988, 1997 , and 2002. ${ }^{1,2}$ The Fact Sheet was designed to provide general insulation information to consumers, including recommended R-values. The information was applicable to both new and existing houses. Related information was provided regarding air sealing and moisture. Before the 1997 update, the Fact Sheet was distributed in paper form to about 40,000 readers/year. The web site received about 500 hits/day between 2002 and 2005. The recommended R-values are also used by a number of other web sites, including those provided by insulation manufacturers and other government energy conservation programs. The purpose of this supporting documentation is to report the revisions that were made in 2008 to the cost and performance databases and to the content of the fact sheet. The updated version was published on the Internet in January of 2008 and is based on the latest available information in mid- to late-2007.

The initial intent of the 2008 Fact Sheet Update was limited in scope, and the main goal was to revise the recommended R-values to reflect current insulation and energy prices. However, comments were solicited from the public during 2006 and 2007 and the results focused attention on several other areas as well. These public comments are summarized in Appendix A and can be found in their entirety on the Building Technology Program web site for envelopes and windows (http://www.govforums.org/E\&W/). On many issues, reviewers provided opposing points of view.

Many comments dealt with a comparison between the recommendations found in the 2006 International Energy Conservation Code (IECC) and those produced for the Fact Sheet by the ZipCode analysis tool. ${ }^{3,4}$ Some reviewers felt that we should rely solely on the IECC values and should not make any other calculations. Other reviewers felt that we should use the IECC values as a lower limit and recommend higher levels whenever they could save energy at a reasonable cost. Still others pointed out that the new IECC climate zone definitions have been broadly adopted by other agencies and that our recommendations should conform to that format as well.

These comments were carefully considered. The 2008 revision reflects the reviewers' input in 
many ways. We have attempted to balance the competing points of view and also to work within the confines of the resources available for the project. All of the cost and performance databases were revised, as described in Section 2. The revised databases are all included in Appendix B. The updated fact sheet text is included in Appendix C

\section{REVISIONS TO THE CALCULATIONS}

\subsection{THE ZIPCODE INSULATION PROGRAM METHODOLOGY}

The DOE Insulation Fact Sheet originally used a computer model called the ZipCode Insulation program, developed by Stephen Peterson at the National Institute of Standards and Technology (NIST) in the 1980s. ${ }^{4}$ This computer program has many strengths, including the use of local values for weather variables, fuel prices, and insulation prices for new and existing homes. However, this same local focus has always made it difficult to summarize the results for broader regions. Also, the calculations rely upon "modified one-dimensional heat transfer" calculations. Whole-house computer models are now the state of the art for evaluating energy conservation options. These whole house models do a better job of reflecting the many inter-related and dynamic energy flows in a structure.

One of the early goals in this revision was to compare the ZipCode's one-dimensional results to a whole house model. In the ZipCode Users Guide, Stephen Petersen describes how the onedimensional savings estimates are based on whole-house correction factors developed for the PEAR program. ${ }^{4}$ Further investigation revealed that these correction factors resulted from a regression analysis of savings due to varying insulation levels using the output from over 10,000 whole house DOE- 2 computer models, covering 45 climates and 5 residential building types. ${ }^{5}$ Although the family of whole-house computer models, including the DOE-2 computer code, has been significantly improved over the last 25 years, the resources to duplicate this extensive modeling effort do not exist in today's budget. Also, one of our reviewers compared the ZipCode results to a modern whole-house model, and we were heartened to learn that the corrected onedimensional methodology produced surprisingly similar results for the cases and climates considered. We therefore elected to continue to use the whole house correction factors developed for the PEAR program. These equations and correction factors are therefore unchanged from those shown in the 1997 Supporting Documentation. ${ }^{6}$

The recommended insulation levels correspond to the insulation level that produces the optimal life-cycle savings for the consumer. (The life-cycle savings balances the current expense for installed insulation against the present value of the future energy savings.) Some reviewers noted that this is a "shallow solution space". That is, there are many answers that produce life cycle savings very close to the optimal savings. Our analysis supported this view, and the results of this approach were helpful in developing the recommendations for the IECC climate regions, which cover a very broad range of energy costs and insulation prices. To select the levels recommended for each region, the ZipCode was processed to calculate the life-cycle savings for a broad range of possible insulation levels. Every insulation level that produced life-cycle savings of at least $95 \%$ of the optimal savings was then output for consideration. For new buildings, the range was then restricted to levels at or greater than the 2006 IECC coderecommended level. For existing buildings, the analysis considered both uninsulated buildings and buildings with some insulation already in place.

Although we considered updating the cooling degree-hour database to more recent data, the newer data bases did not include cooling hours relative to the $74^{\circ} \mathrm{F}$ base used in the derivation of 
the correction factors. However, we were able to update the heating degree-days to more current climatic data. ${ }^{7}$ The postal service has reassigned a number of the 3-digit level zip codes to new areas and has begun to use some of the numbers that were previously reserved. Appendix B includes a full list of the zip-code specific climate and cost factor data used in this update.

\subsection{PERFORMANCE ASSUMPTIONS}

The thermal performance of most insulation measures was unchanged from previous editions of the fact sheet, with some changes for foam insulation based on recent formulations. Also, the Ufactors for both wood and metal-framed walls were revised based on research that has been completed during the last 10 years, including both experimental and finite difference analyses. ${ }^{8,9}$ Furthermore, ASHRAE has recently reassessed wall framing factors in a detailed survey effort. ${ }^{10}$ The California Energy Commission extended that effort to the state of California. ${ }^{11}$ Both studies found that approximately $25 \%$ of the wall area, net of the window area, was comprised of framing. The experimental wall heat transfer work found a roughly one to one correspondence between the framing factor and the difference between the nominal and the whole wall performance for wood-framed walls. The wood wall U-values were adjusted accordingly as summarized in Table 1. The experimental results for multiple metal wall framing arrangements were used to estimate the framing loss for these systems and the resulting heat transfer coefficients are summarized in Appendix B.

For wood-framed attics, a parallel path heat transfer model was used to include the heat transfer through the framing members in the estimate of the overall U-values. For metal-framed attics, the U-values were calculated based on experimental and modeling work, using the more conservative of the values for either a truss system or a joist and rafter system. ${ }^{12}$ The thermal performance of cathedral ceilings and band joists were unchanged. These U-factors are summarized in Appendix B.

For foundation insulation measures, including basements, floors, crawl spaces, and slab edges, the thermal performance was unchanged from previous editions of the fact sheet. The savings factors, based on an extensive finite difference analysis for multiple climates, are summarized in Appendix B. ${ }^{13}$

Table 1 Summary of wall U-value factors

\begin{tabular}{|l|l|}
\hline Outside film coefficient & $0.17 \mathrm{~h}-\mathrm{ft}^{2}-{ }^{\circ} \mathrm{F} / \mathrm{Btu}$ \\
\hline Inside film coefficient & $0.68 \mathrm{~h}-\mathrm{ft}^{2}-{ }^{\circ} \mathrm{F} / \mathrm{Btu}$ \\
\hline Gypsum & $0.45 \mathrm{~h}-\mathrm{ft}^{2}-{ }^{\circ} / \mathrm{Btu}$ \\
\hline Cavity insulation & Rated R-value $\left(\right.$ or $0.9 \mathrm{~h}-\mathrm{ft}^{2}-{ }^{\circ} \mathrm{F} / \mathrm{Btu}$ for an empty cavity) \\
\hline Plywood & $1.32 \mathrm{~h}-\mathrm{ft}^{2}-{ }^{\circ} \mathrm{F} / \mathrm{Btu}$ \\
\hline Siding & $0.81 \mathrm{~h}-\mathrm{ft}^{2}-{ }^{\circ} \mathrm{F} / \mathrm{Btu}$ \\
\hline Total Reduced Clear Wall & $0.75{ }^{*}$ Sum above [metal-framed walls: $\left.0.40 * \mathrm{Sum}\right]$ \\
\hline Foam Sheathing & Rated R-value $\left(\mathrm{h}-\mathrm{ft}^{2}-{ }^{\circ} \mathrm{F} / \mathrm{Btu}\right)$ \\
\hline Total R & Sum Reduced Clear Wall + foam sheathing \\
\hline Total U & $1 /$ Total R \\
\hline
\end{tabular}

The performance of heating and cooling systems was updated to reflect technology and market improvements, as summarized in Table 2. 
Table 2 Heating and cooling system performance assumption changes

\begin{tabular}{|l|l|}
\hline New electric air conditioner seasonal energy efficiency ratio (SEER), BtuW-h & 13 \\
\hline Existing electric air conditioner SEER, Btu/W-h & 9.7 \\
\hline $\begin{array}{l}\text { New heat pump heating seasonal performance factor (HSPF) used in Eq. 1 in } \\
\text { Ref. 6, Btu/W-h }\end{array}$ & 7.7 \\
\hline Existing heat pump HSPF , Btu/W-h & 6.6 \\
\hline New heat pump SEER, Btu/W-h & 13. \\
\hline Existing heat pump SEER, Btu/W-h & 9.7 \\
\hline & \multicolumn{2}{|l|}{} \\
\hline
\end{tabular}

\subsection{COST ASSUMPTIONS}

Based upon comments, economic lifetimes have been extended to 40 years for new houses and 30 years for existing houses. The previous values were 30 years for new houses and 20 years for existing houses. (In fact, this change made little difference in the results because the additional ten years of savings occurs far in the future and are therefore heavily discounted in the savings calculation.)

Current energy prices, on a state-average basis, come from the Energy Information Administration (EIA), as in previous editions of the fact sheet. ${ }^{14}$ For natural gas, fuel oil, and propane, the price represents the average retail price from October 2006 to March 2007. For electricity, summer prices from 2006 were used. The National Institute of Standards and Technology provides escalation factors for energy. ${ }^{15}$ These energy prices and escalation factors are all summarized in Appendix B. A number of reviewers objected to the near-level nature of the official energy price projections, arguing that energy prices are likely to rise significantly. However, we are interested in the increase in price relative to general inflation, and much of the general inflation index is driven by energy costs. Also, we doubted that any other crystal ball would be demonstrably better than the National Energy Modeling System used by the DOE to develop the projections. (The reader may be interested in looking at the EIA's own assessment of its predictions vs. reality. ${ }^{16}$ )

Because the Fact Sheet analysis has always been focused on finding the optimal life-cycle savings, the least cost option that provides a given level of thermal performance is used in the database. That is, if two products provide the same R-value but have different costs, the lower cost is used. It also follows that the database will not include an option for a given R-value if a higher R-value is available at a lower cost. The results have never specified products by name or material, only by R-value.

Prior to 1997, insulation cost data were based on a 1986 survey by the National Association of Home Builders (NAHB). The NAHB survey defined costs for insulation measures in new construction only, but was also used to represent retrofit costs. Because insulation amounts often increase in discrete steps, the recommendations for insulation are very sensitive to the cost assumptions used in the life-cycle analysis. A sensitivity study was made in 1995 varying the cost of insulation by $\pm 20 \%$. This changed the recommended level of insulation by a factor of two in many parts of the country. Given this sensitivity, a survey of insulation costs was commissioned as a part of the 1997 revision to the fact sheet. ${ }^{17}$ That survey, which began with the lofty goal of obtaining regional data from a large number of installers, cost $\sim \$ 100 \mathrm{~K}$, and in the end received complete survey results from a relatively small number of contractors, even after the survey program was modified to reimburse the contractors for their time. That survey 
solicited separate estimates for retrofit and new construction.

There were five main sources for the cost data used in the 2008 Fact Sheet update:

- Original database used in development of the 1988 Fact Sheet: ("Costs were developed from the NAHB/RF's cost data base and verified by telephone calls to local builders, subcontractors, and building material suppliers." $)^{18}$

- Survey performed for the 1997 update to the Insulation Fact Sheet: approximately 30 contractors and 18 builders responded, ${ }^{17}$

- Database for Energy Efficient Resources (DEER), California Public Utilities Commission, 2005 Update, ${ }^{19}$

- RS Means Building Construction Cost Data, 2007 (which was also the source for historical escalation factors and zip-code specific city cost factors),

- Informal review of insulation material prices on the Internet and by phone during the summers of 2006 and 2007, and

- BNi Building News Home Builder's 2008 Costbook and BNi Building News Home Remodeler's 2008 Costbook $^{20,21}$ (provided a point of comparison between new construction and retrofit applications).

Each of these sources provided useful, if limited, information. The original database had arguably the best resource of contractor and builder price information, but is now almost 20 years old. The 1996 survey was ambitious in intent, but received responses from a relatively small number of contractors and builders, and is now 10 years old. In 1997, the Fact Sheet was updated using a combination of the 1988 and 1996 surveys. These prices were updated to 2007 using the R. S. Means historical cost factor, and are referred to here as the "1997 Fact Sheet Updated". The DEER database is more current, but reflects California prices, which tend to be 5 to $20 \%$ greater than the national average based upon the R.S. Means city cost indices. Also, the DEER documentation is not specific about the ultimate source of the data provided in the DEER database for envelope measures. The R.S. Means database is comprehensive, but is based upon union labor costs for large building projects and many reviewers have noted that its prices are significantly higher than typical residential construction costs. Considering the wide diversity in these sources, they were surprisingly often in the same ballpark, as shown in Table 3 . The phone review of materials prices summarized in Table 4 is a useful touch point, but provides retail prices, higher than the prices that would be paid by many contractors.

The prices used in this update are shown in Appendix B and were based upon the following considerations:

Location Indices: The RS Means city cost indices are current and specific to thermal and moisture protection.

Historical Cost Factors: The RS Means historical cost factors are based on national averages and are not specific to thermal and moisture protection. The R. S. Means historical update factor from 1996 to 2007 was 1.5 .

Attics: The contractors provided blown-in loose fill data in 1996 which, when updated to 2007, was within a few cents of the DEER database. The RS Means values were $42 \%$ to $67 \%$ higher for comparable R-values. The updated 1996 values were selected.

Cathedral Ceilings: The contractors provided installed batt prices in 1996. There is no comparable entry in the DEER database. The RS Means values for blown-in open-celled foam are more than 10 times higher on a per R-value basis than the updated batt prices. Therefore, the updated 1996 values were selected. 
Walls: In 1996, contractors provided information for batts used in combination with foam sheathing boards. The R13, R15, and R21 products were new and had premium prices at that time. This is demonstrated by comparing the updated prices for these insulation levels to the 2005 DEER prices for California, as shown in Table 3. A comparison of retail prices for a single brand in 2007 showed a $\$ 0.05$ premium for R13 compared to R11, not the escalated difference of $\$ 0.09$. The price for R13 was therefore conservatively set at $\$ 0.51$, six cents more than R11 and ten cents less than R15. For R15 and the R5/R13 combination, the DEER prices of $\$ 0.61$ and $\$ 1.37$, respectively, were used. For the thicker walls, the DEER prices don't include the extra cost of framing as do the escalated 1996 values. The polyisocyanurate foam board was not included in the previous data base. For this level, the price premium of $\$ 0.08 / \mathrm{ft}^{2}-\mathrm{R}$ found in the material survey was used to determine the price differential between the extruded polystyrene (XPS) at R5 and the polyiso at R6. The mass wall prices are based on the assumed placement of expanded polystyrene (EPS) between concrete block and a brick façade (unchanged approach since 1986). Other mass wall options were considered, but it's problematical to separate out the cost of the wall from the cost of the insulation system, as was carefully done by the NAHB in 1986. Structural Insulated Panels (SIP) and Exterior Insulation and Finish Systems (EIFS) were also examined. Using R. S. Means prices, an attempt was made to determine the incremental costs for these two wall systems beyond the traditional insulated wall cost. The results were higher than the other wall insulation options for comparable R-values, and so were not included here, although these two options are included in the design options portion of the fact sheet.

Floors: The updated 1996 prices were slightly less than the DEER prices and were selected. R30 was not considered in 1996, so the DEER price was used for that level.

Slab Edge: The updated 1996 prices were used for R4 and R8 for the 2-foot depth. These prices were used, along with the current retail material prices, to estimate the installation cost. This cost, was used along with increased material costs to estimate the R12 price. For the 4foot depths, it was assumed that both the installation and material costs would double.

Crawl Space Walls, Basement Walls, and Band Joists: These measures were not included in the DEER or R. S. Means data bases so the updated 1996 values were used. The premium for $\mathrm{R} 13$ in a crawl space was only $\$ 0.06 / \mathrm{ft}^{2}$ above $\mathrm{R} 11$, so that price was used unchanged. For crawl spaces, the premium for R15 was much higher, so that was reduced to a price difference of $\$ 0.10 / \mathrm{ft}^{2}$ relative to $\mathrm{R} 13$ (consistent with the price differential for walls). R25 was not included in 1996 , so that price was calculated by adding $\$ 0.12 / \mathrm{ft}^{2}$ to the $\mathrm{R} 19$ price, based on an average cost of $\$ 0.02 / \mathrm{ft}^{2}-\mathrm{R}$ for the batt products. For crawl spaces, the coverage was quoted for a four-foot high wall with the insulation extending two feet over the ground (i.e., six square feet for each foot of perimeter length). For basements, the height is assumed to be eight feet, and the cost of framing to accommodate the insulation is included, but the final layer of wallboard or paneling is not. A batt product with a special facing is also included for unfinished basements. An Internet examination found batt products rated at R3 and R6 to fit within furring strip dimensions, but no prices were available so these were not included.

\section{SUMMARY OF REVISIONS TO THE INSULATION FACT SHEET}

Traditionally, the Fact Sheet's role has been to provide the best guidance possible for the individual homeowner. That goal has shaped the calculation approach - which has been to find the insulation level that offers the optimal life cycle cost for a particular homeowner, considering their own fuel costs and other factors. There were always major differences between the 
calculations used to develop code requirements and those that produce the Fact Sheet's recommendations. Most importantly, the code values are generic with respect to fuel, are focused on new construction, and provide values for broad climatic regions. After considering these factors, we decided to continue to provide recommendations based upon specific fuels and local insulation costs, including variations between costs for new and retrofit installations. This process can provide supplemental information to the individual homeowner beyond that needed for code compliance.

The old 3-layered tables used in the printed version of the fact sheet, that called for using your zip code, house type, and fuel type to identify your "insulation zone" are gone. Most readers will either use the Internet's active look-up table for their zip code or will run the interactive Java version of the ZipCode computer model. Ranges of recommended R-values were prepared for those who need a quick visual summary consistent with the IECC climate zones.

There were many requests to expand the scope of the Insulation Fact Sheet. But we were concerned that the document was in danger of morphing from a consumer-friendly fact sheet into a less useful reference book. To address both the need to provide information about related topics, such as super-efficient homes, and to restrict the overall size of the fact sheet, we expanded the use of web links to other DOE web sites. Thus, the discussion of air sealing and moisture was reduced somewhat, but links were added to other fact sheets that provide much more extensive and detailed information on these topics.

To improve the usability of the web site, the material was more clearly separated into information for existing homes and information for new homes. A new section was added to discuss design options for new homes, including structural insulated panels, insulated concrete forms, massive construction techniques, and cathedralized attics. Insulation materials that were not on the market in 1997, such as icynene foam, were added.

Using the email links provided at the bottom of each fact sheet web page, consumers contact us on a regular basis. We'll be watching this feedback over the next several months to assess whether any further changes or modifications are needed to meet the needs of the fact sheet readers. 
Table 3 Comparison of selected installed costs for insulation measures in new houses

\begin{tabular}{|c|c|c|c|c|}
\hline $\begin{array}{c}\text { Sheathing } \\
\text { Insulation R- } \\
\text { Value (h- } \mathrm{ft}^{2}- \\
\left.{ }^{\circ} \mathrm{F} / \mathrm{Btu}\right)\end{array}$ & $\begin{array}{c}\text { Cavity } \\
\text { Insulation R- } \\
\text { Value (h- } \mathrm{ft}^{2}- \\
\left.{ }^{\circ} \mathrm{F} / \mathrm{Btu}\right) \\
\end{array}$ & $\begin{array}{c}1997 \text { Fact Sheet } \\
\text { Updated to } \\
2007\end{array}$ & DEER & RS MEANS \\
\hline \multicolumn{5}{|c|}{ Attic } \\
\hline & 11 & $\$ 0.36$ & & $\$ 0.60$ \\
\hline & 19 & $\$ 0.50$ & $\$ 0.62$ & $\$ 0.71$ \\
\hline & 30 & $\$ 0.68$ & $\$ 0.76$ & \\
\hline & 38 & $\$ 0.84$ & $\$ 0.86$ & $\$ 1.53$ \\
\hline & 49 & $\$ 1.02$ & $\$ 0.86$ & \\
\hline \multicolumn{5}{|c|}{ Floor } \\
\hline & 13 & $\$ 0.62$ & $\$ 0.69$ & \\
\hline & 19 & $\$ 0.80$ & $\$ 0.89$ & \\
\hline \multicolumn{5}{|c|}{ Wall } \\
\hline & 11 & $\$ 0.45$ & & $\$ 0.70$ \\
\hline & 15 & $\$ 0.84$ & $\$ 0.61$ & \\
\hline & 19 & $\$ 1.91$ & $\$ 0.65(\mathrm{a})$ & $\$ 0.81(a)$ \\
\hline & 21 & $\$ 2.07$ & $\$ 0.68(\mathrm{a})$ & \\
\hline 5 & 13 & $\$ 1.41$ & $\$ 1.37$ & \\
\hline 5 & 19 & $\$ 2.78$ & $\$ 1.56(\mathrm{a})$ & \\
\hline 5 & 21 & $\$ 2.94$ & $\$ 1.84(\mathrm{a})$ & \\
\hline \multicolumn{5}{|c|}{ Mass wall } \\
\hline 3.8 & & $\$ 0.33$ & & $\$ 0.87$ or 2.16 \\
\hline 7.6 & & $\$ 0.78$ & & $\$ 3.74$ \\
\hline \multicolumn{5}{|c|}{ Optimal Value Engineering wall } \\
\hline 2.5 & 19 & $\$ 1.37$ & & \\
\hline 5 & 19 & $\$ 1.53$ & $\$ 1.56$ & \\
\hline \multirow[t]{3}{*}{5} & 21 & $\$ 1.70$ & $\$ 1.84$ & \\
\hline & 19 & $\$ 0.66$ & $\$ 0.65$ & \\
\hline & 21 & $\$ 0.83$ & $\$ 0.68$ & \\
\hline
\end{tabular}

(a) These costs don't appear to include the extra framing costs associated with the greater wall depth. 
Table 4 Retail material costs in 31 states reviewed in 2006 and 2007

\begin{tabular}{|c|c|c|c|}
\hline \multirow[t]{2}{*}{ Material } & \multirow{2}{*}{$\begin{array}{c}\text { Number of data } \\
\text { points }\end{array}$} & \multicolumn{2}{|c|}{$\$ / \mathrm{ft}^{2}-\mathrm{R}$ (standard deviation) } \\
\hline & & 2006 & 2007 \\
\hline \multicolumn{4}{|c|}{ Fiberglass batts } \\
\hline R11 & 1 & & $\$ 0.034$ \\
\hline R13 & 28 in 2006,3 in 2007 & $\$ 0.027(\$ 0.005)$ & $\$ 0.030(\$ 0.005)$ \\
\hline R15 & 1 & & $\$ 0.031$ \\
\hline R19 & 3 & $\$ 0.025(\$ 0.001)$ & \\
\hline $\mathrm{R} 30$ & 4 & $\$ 0.017(\$ 0.009)$ & \\
\hline \multicolumn{4}{|c|}{ Extruded polystyrene } \\
\hline 0.5 in. & 16 & $\$ 0.114(\$ 0.030)$ & \\
\hline 0.5 in foil-faced & 1 & $\$ 0.197$ & \\
\hline 0.75 in. foil-faced & 1 & $\$ 0.097$ & \\
\hline 0.75 in. & 2 & $\$ 0.092$ & \\
\hline 1.5 in. & 1 & $\$ 0.088$ & \\
\hline \multicolumn{4}{|c|}{ Polyisocyanurate } \\
\hline $.5 \mathrm{in}$. foil-faced & 4 & $\$ 0.172(\$ 0.058)$ & \\
\hline 1 in. foil-faced & 1 & $\$ 0.186$ & \\
\hline $2 \mathrm{in}$. foil-faced & 1 & $\$ 0.161$ & \\
\hline \multicolumn{4}{|c|}{ Expanded polystyrene } \\
\hline 0.75 in. R2.9 & 1 & & $\$ 0.113$ \\
\hline 1 in. R3.9 & 1 & & $\$ 0.095$ \\
\hline 2 in. R7.8 & 1 & & $\$ 0.090$ \\
\hline \multicolumn{4}{|c|}{ Cellulose } \\
\hline & 22 & $\$ 0.011(\$ 0.003)$ & \\
\hline
\end{tabular}




\section{REFERENCES}

${ }^{1}$ Insulation Fact Sheet, DOE/CE-0180, January 1988, Department of Energy, Assistant Secretary Conservation and Renewable Energy, U.S. Government Printing Office, Washington, DC

${ }^{2}$ Insulation Fact Sheet, DOE/CE-0180, August, 1997, Department of Energy, Assistant Secretary Conservation and Renewable Energy, U.S. Government Printing Office, Washington, DC (http://www.ornl.gov/roofs+walls)

${ }^{3}$ International Code Council, International Energy Conservation Code 2006, January 2006

${ }^{4}$ Stephen R. Petersen, ZIP - The ZIP-Code Insulation Program (Version 1.0) Economic Insulation Levels for New and Existing Houses by Three-Digit ZIP Code, Users Guide and Reference Manual, ORNL/TM-1109, NISTIR 88-3801, Oak Ridge National

Laboratory, Oak Ridge, TN, January 1989

5 J. Huang, R. Ritschard, J. Bull, S. Byrne, and I. Turiel, Affordable Housing Through Energy Conservation, A Guide to Designing and Constructing Energy Efficient Homes, Technical Support Document, DOE/SF/0098-H3, Volume 2 of 3, U.S. Department of Energy, June 1989

${ }^{6}$ T. K. Stovall, Supporting Documentation for the1997 Revision to the DOE Insulation Fact Sheet, ORNL-6907, Oak Ridge National Laboratory, August 1997

${ }^{7}$ Climatography of the United States No. 81, Supplement No. 2, Annual Degree Days to Selected Bases 1971-2000, National Oceanic and Atmospheric Administration, June 20, 2002

${ }^{8}$ J. Kosny, P. W. Childs J.A. Atchley, "Steady-State Thermal Performance Evaluation of Wood and Steel-Framed Wall Assemblies," Private Project Report, April, 2006, Oak Ridge National Laboratory

9 J. Kosny, D. Yarbrough, P. Childs, S. A. Mohiuddin, "How the Same Wall Can Have Several Different R-Values - Relations Between the Amount of Framing and the Overall Thermal Performance in Wood and Steel-Framed Walls," to be published by ASHRAE in the Proceedings for the Building X Conference, Clearwater Beach, December 2007

${ }^{10}$ Carpenter, S.C., and Schumacher C., "Characterization of Framing Factors for Wood-Framed Low-Rise Residential Buildings," ASHRAE Transactions Vol. 109, Pt. 1, Feb 2003

${ }^{11}$ Enermodal Engineering Limited, "Characterization of Framing Factors for Low-Rise Residential Building Envelopes in California", P500-02-002, California Energy Commission, December 2001, http://cacx.org/reports/2002-02-15 500-02-002.PDF

${ }^{12}$ Petrie, T.W., Kosny, J., Atchley, J. A., and Desjarlais, A. O., "Effect of Steel Framing in Attic/Ceiling Assemblies on Overall Thermal Resistance," Insulation Materials: Testing and Applications: Fourth Volume, ASTM STP 1426, A. O. Desjarlais and R. R. Zarr, Eds., American Society for Testing and Materials, West Conshohocken, PA 2002.

${ }^{13}$ J. Christian and W. Strzepek, "Procedure for Determining the Optimum Foundation Levels for New, Low-Rise Residential Buildings,” ASHRAE Transactions, Vol. 93, Part 1, 1987

${ }^{14}$ Current energy prices are available at www.eia.doe.gov

${ }^{15}$ A. S. Rushing and B. C. Lippiatt, Energy Price Indices and Discount Factors for Life-Cycle Cost Analysis - April 2007, NISTIR 85-3273-22 (Rev 5/07) Annual Supplement to NIST Handbook 135 and NBS Special Publication 709

${ }^{16}$ Annual Energy Outlook Retrospective Review: Evaluation of Projections in Past Editions (1982-2006) DOE/EIA-0640(2006) April 2007, www.eia.doe.gov

${ }^{17}$ T. K. Stovall, Supporting Documentation for the1997 Revision to the DOE Insulation Fact Sheet, ORNL-6907, Oak Ridge National Laboratory, August 1997

${ }^{18}$ NAHB National Research Center, ASHRAE Research Project 494-RP, An Economic Data Base in Support of SPC 90.2: Costs of Residential Energy, Thermal Envelope and HVAC 
Equipment, Dec 1, 1986

${ }^{19}$ Itron, Inc., et al., 2004-2005 Database for Energy Efficiency Resources (DEER) Update Study Final Report, December 2005, http://eega.cpuc.ca.gov/deer/

${ }^{20}$ BNi Building News Home Builder's 2008 Costbook, Sixteenth Edition

${ }^{21}$ BNi Building News Home Remodeler's 2008 Costbook, Sixteenth Edition 


\section{APPENDIX A PUBLIC REVIEW COMMENTS}

The following tables summarize the comments received.

\section{Table 5 Presentation Issues}

Completely re-write and improve focus on consumer - add more practical advice - make it shorter. Convert into separate brochures, one for new and one for existing houses.

Add more regarding air infiltration and air sealing, comfort issues - refer to Weatherization and Building America programs. Add 'performance - based compliance to construction'.

Add more warnings about poor installations

Point out that insulation is typically enclosed and difficult to upgrade at a later date.

Get rid of awkward tables

Add embodied energy issues. Talk about environmental benefits, emissions savings in terms of equivalent car miles driven.

Improve comparisons between material types: compression, combustibility, air sealing. Add more information regarding low-density foam and cellulose, Eliminate mention of R-11 and R15 batts, De-emphasize fiberglass.

Update to include recent research on cathedralized/unvented attics

Revise or eliminate references

Moisture section - make it shorter while adding more 'actionable items'

De-emphasize R-value. Focus on super-efficient homes, especially use of cellulose, but don't go as far as zero energy homes, add information on HVAC sizing, duct placement, windows, radon, IAQ, etc.

\section{Table 6 Code Related Issues}

Stick with code R-values

Use code as minimum R-value considered

Comparison of 03 to current codes that shows many reduced levels due to combination of more climate zones into fewer climate zones

Don't increase above code R-values

Use DOE, IECC, and International Residential Code climate zones

\section{Table 7 Economic Calculation Issues}

Gather new cost data from more contractors, include sub-state price variability (urban, rural), consider future price decreases due to increased market penetration

If we don't gather new information, update old

Update fuel prices

Report results for ranges of energy and insulation costs - then make max R the recommended level

Account for future increases in insulation installation cost as an avoided cost now.

Assign value to future emissions reductions (use European Kyoto credit value)

Savings due to reducing HVAC-duct system size

Higher discount rate, longer lifetime (50 years), add energy price taxes, demand charges, etc., 
Consider relative magnitude of energy price increases and insulation price

Use statistically mixed bag of fuel types and house

Change assumptions to produce a "high efficiency" home; do not use assumptions that produce results similar to code. Points to Energy Star that uses codes as 'base'.

Add windows glazing and related impact on recommended R-values

Perform independent projection of fuel price increases instead of using DOE projections

Table 8 Thermal Calculation Issues

Use best models available to achieve highest accuracy and apply to statistically defined house type and fuel

Continue to use 1-D models

Use better models to more properly represent massive systems. New models show that summer energy penalties outweigh winter savings for basement insulation in many zones.

Don't evaluate R-15 high density batts because they are only produced by fiberglass

manufacturers

Change the assumed duct location to inside the envelope.

Credit foam and cellulose with air sealing so lower R-value has added value 
Table 9 Insulation cost and performance parameters used for 2008 fact sheet analysis for above-ground measures

\begin{tabular}{|c|c|c|c|}
\hline $\begin{array}{c}\text { Cavity Insulation } \\
\text { R-Value (h- } \mathrm{ft}^{2}- \\
\left.{ }^{\circ} \mathrm{F} / \mathrm{Btu}\right)\end{array}$ & $\begin{array}{c}\text { Sheathing } \\
\text { Insulation R-Value } \\
\left(\mathrm{h}-\mathrm{ft}^{2}-{ }^{\circ} \mathrm{F} / \mathrm{Btu}\right)\end{array}$ & $\begin{array}{c}\text { Total Composite U- } \\
\text { Value }\end{array}$ & $\begin{array}{c}\text { Cost, new } \\
\text { construction } \\
\text { (retrofit)\$/ft }\end{array}$ \\
\hline \multicolumn{4}{|c|}{ Wood-framed attic, Base U-Value $=0.254$} \\
\hline 11 & & 0.0688 & $0.36(0.41)$ \\
\hline 19 & & 0.0455 & $0.50(0.56)$ \\
\hline 30 & & 0.0333 & $0.68(0.74)$ \\
\hline 38 & & 0.0241 & $0.84(0.90)$ \\
\hline 49 & & 0.0199 & $1.02(1.16)$ \\
\hline 60 & & 0.0193 & $1.23(1.37)$ \\
\hline \multicolumn{4}{|c|}{ Cathedral Ceiling, Base U-Value $=0.2616$} \\
\hline 13 & & 0.0666 & 0.62 \\
\hline 15 & & 0.0607 & 0.72 \\
\hline 19 & & 0.0493 & 0.80 \\
\hline 22 & & 0.0434 & 0.93 \\
\hline 30 & & 0.0332 & 1.10 \\
\hline 38 & & 0.027 & 1.31 \\
\hline 49 & & 0.0216 & 3.03 \\
\hline 60 & & 0.0178 & 3.44 \\
\hline \multicolumn{4}{|c|}{ Wood-framed wall, Base U-Value $=0.3079$} \\
\hline 13 & & 0.0812 & $0.51(1.32)$ \\
\hline 15 & & 0.0723 & 0.61 \\
\hline 13 & 2.5 & 0.0675 & 1.21 \\
\hline 15 & 2.5 & 0.0613 & 1.28 \\
\hline 13 & 5 & 0.0577 & 1.37 \\
\hline 15 & 5 & 0.0531 & 1.43 \\
\hline 15 & 6 & 0.0504 & 1.91 \\
\hline 21 & 2.5 & 0.048 & 2.64 \\
\hline 21 & 5 & 0.0429 & 2.81 \\
\hline \multirow{4}{*}{21} & 6 & 0.0411 & 3.29 \\
\hline & 2.5 & 0.1480 & $(0.76)$ \\
\hline & 5 & 0.1210 & $(0.92)$ \\
\hline & 6 & 0.1160 & $(1.40)$ \\
\hline 11 pre-existing & 2.5 & 0.0700 & $(0.76)$ \\
\hline 11 pre-existing & 5 & 0.0630 & $(0.92)$ \\
\hline 11 pre-existing & 6 & 0.0620 & $(1.40)$ \\
\hline \multicolumn{4}{|c|}{ Optimum Value Engineered Wall, Base U-Value $=0.3079$} \\
\hline 19 & & 0.0662 & 0.65 \\
\hline 21 & & 0.0546 & 0.68 \\
\hline
\end{tabular}




\begin{tabular}{|c|c|c|c|}
\hline $\begin{array}{c}\text { Cavity Insulation } \\
\text { R-Value (h-ft' }{ }^{2}- \\
\left.{ }^{\circ} \mathrm{F} / \mathrm{Btu}\right)\end{array}$ & $\begin{array}{c}\text { Sheathing } \\
\text { Insulation R-Value } \\
\left(\mathrm{h}-\mathrm{ft}^{2}-{ }^{\circ} \mathrm{F} / \mathrm{Btu}\right)\end{array}$ & $\begin{array}{c}\text { Total Composite U- } \\
\text { Value }\end{array}$ & $\begin{array}{c}\text { Cost, new } \\
\text { construction } \\
\text { (retrofit) } \$ / \mathbf{f t}^{2}\end{array}$ \\
\hline 21 & 2.5 & 0.048 & 1.40 \\
\hline 21 & 5 & 0.0429 & 1.53 \\
\hline 21 & 6 & 0.0411 & 2.18 \\
\hline \multicolumn{4}{|c|}{ Mass wall insulation, Base U-Value $=0.2525$} \\
\hline 3.9 & & 0.1437 & $1.06(1.11)$ \\
\hline 7.8 & & 0.0921 & $1.41(1.46)$ \\
\hline 11.7 & & 0.0678 & $1.76(1.81)$ \\
\hline 15.6 & & 0.0536 & $2.11(2.16)$ \\
\hline \multicolumn{4}{|c|}{ Metal-framed attic, Base U-Value $=0.592$} \\
\hline 11 & & 0.386 & $0.36(0.41)$ \\
\hline 19 & & 0.278 & $0.50(0.56)$ \\
\hline 30 & & 0.047 & $0.68(0.74)$ \\
\hline 38 & & 0.034 & $0.84(0.90)$ \\
\hline 49 & & 0.025 & $1.02(1.16)$ \\
\hline 38 & 5 & 0.025 & 1.71 \\
\hline 49 & 5 & 0.020 & 1.89 \\
\hline 49 & 10 & 0.018 & 2.30 \\
\hline \multicolumn{4}{|c|}{ Metal-framed wall, Base U-Value $=0.5774$} \\
\hline 0 & 2.5 & 0.2363 & $0.71(0.76)$ \\
\hline 0 & 5 & 0.1485 & $0.87(0.92)$ \\
\hline 13 & 2.5 & 0.1102 & 1.21 \\
\hline 15 & 2.5 & 0.1013 & 1.28 \\
\hline 13 & 5 & 0.0864 & 1.37 \\
\hline 15 & 5 & 0.0808 & 1.43 \\
\hline 13 & 6 & 0.0795 & 1.85 \\
\hline 15 & 6 & 0.0748 & 1.91 \\
\hline 13 & 10 & 0.0603 & 2.23 \\
\hline 15 & 10 & 0.0576 & 2.25 \\
\hline 19 & 10 & 0.0554 & 3.65 \\
\hline 21 & 10 & 0.0506 & 3.68 \\
\hline 13 & & 0.1522 & $(1.32)$ \\
\hline 11 pre-existing & 2.5 & 0.1209 & $(0.76)$ \\
\hline 11 pre-existing & 5 & 0.0928 & $(0.92)$ \\
\hline 11 pre-existing & 6 & 0.0849 & $(1.40)$ \\
\hline \multicolumn{3}{|c|}{ Band joist insulation, Base U-Value $=0.197$} & $\begin{array}{c}\text { Cost, } \\
\text { \$/ linear foot }\end{array}$ \\
\hline 13 & & 0.059 & 0.54 \\
\hline 19 & & 0.044 & 0.68 \\
\hline 30 & & 0.031 & 0.90 \\
\hline
\end{tabular}


Table 10 Insulation cost used for $\mathbf{2 0 0 8}$ fact sheet analysis for foundation measures

\begin{tabular}{|c|c|c|c|c|}
\hline \multirow[b]{2}{*}{ R-Value $\left(\mathrm{h}-\mathrm{ft}^{2}-{ }^{\circ} \mathrm{F} / \mathrm{Btu}\right)$} & \multicolumn{2}{|c|}{ Energy saving factors ${ }^{\mathrm{a}}$} & \multirow{2}{*}{$\begin{array}{c}\begin{array}{c}\text { New } \\
\text { construction }\end{array} \\
\$ / \mathbf{f t}^{2}\end{array}$} & \multirow{2}{*}{$\begin{array}{c}\text { Retrofit } \\
\$ / \mathbf{f t}^{2}\end{array}$} \\
\hline & $\beta_{\mathrm{h}}$ & $\beta_{\mathrm{c}}$ & & \\
\hline \multicolumn{5}{|l|}{ Wood floor } \\
\hline 13 & 1.8 & 0.12 & 0.62 & 0.69 \\
\hline 19 & 1.96 & 0.12 & 0.80 & 0.87 \\
\hline 25 & 2.12 & 0.12 & 0.93 & 1.14 \\
\hline 30 & 2.22 & 0.12 & 1.34 & 1.34 \\
\hline \multicolumn{5}{|l|}{ Metal-framed floor } \\
\hline 13 & 1.14 & 0.07 & 0.62 & 0.69 \\
\hline 19 & 1.33 & 0.09 & 0.80 & 0.87 \\
\hline 25 & 1.45 & 0.1 & 0.93 & 1.14 \\
\hline \multirow[t]{2}{*}{30} & 1.52 & 0.11 & 1.34 & 1.34 \\
\hline & & & $\begin{array}{c}\text { \$/linear foot of } \\
\text { perimeter }\end{array}$ & $\begin{array}{c}\text { \$/linear foot of } \\
\text { perimeter }\end{array}$ \\
\hline \multicolumn{5}{|l|}{$\underline{\text { Slab edge }}$} \\
\hline $4,2 \mathrm{ft}$ & 2.79 & 0.23 & 2.10 & \\
\hline $8,2 \mathrm{ft}$ & 3.25 & 0.25 & 2.73 & \\
\hline $12,2 \mathrm{ft}$ & 3.53 & 0.25 & 3.36 & \\
\hline $4,4 \mathrm{ft}$ & 3.56 & 0.23 & 4.20 & \\
\hline $8,4 \mathrm{ft}$ & 4.25 & 0.25 & 5.46 & \\
\hline $12,4 \mathrm{ft}$ & 4.72 & 0.25 & 6.72 & \\
\hline \multicolumn{5}{|l|}{ Crawl space wall } \\
\hline 13 & 22.2 & 0.94 & 3.69 & 3.96 \\
\hline 15 & 22.5 & 0.93 & 4.29 & 4.56 \\
\hline 19 & 22.9 & 0.94 & 4.68 & 4.95 \\
\hline 25 & 23.3 & 0.96 & 5.40 & 5.67 \\
\hline \multicolumn{5}{|l|}{ Basement exterior } \\
\hline 4 & 28.57 & 0.45 & 9.30 & \\
\hline 5 & 30 & 0.47 & 10.52 & \\
\hline 8 & 32.75 & 0.5 & 13.95 & \\
\hline 10 & 34 & 0.51 & 16.31 & \\
\hline 12 & 35.03 & 0.52 & 18.45 & \\
\hline 15 & 35.35 & 0.54 & 21.83 & \\
\hline \multicolumn{5}{|l|}{$\frac{\text { Basement interior }}{\text { finished }}$} \\
\hline 11 & 34.24 & 0.51 & 9.72 & 10.12 \\
\hline 13 & 34.59 & 0.51 & 10.80 & 11.20 \\
\hline 15 & 34.85 & 0.52 & 11.60 & 12.00 \\
\hline 19 & 35.2 & 0.52 & 13.95 & 14.35 \\
\hline 25 & 35.8 & 0.53 & 14.91 & 15.31 \\
\hline \multicolumn{5}{|l|}{$\frac{\text { Basement interior }}{\text { unfinished }}$} \\
\hline 111 & 34.24 & 0.51 & 6.36 & 6.41 \\
\hline
\end{tabular}


Table 11 Residential Energy Prices for 2008 Update

\begin{tabular}{|c|c|c|c|c|}
\hline State & $\begin{array}{c}\text { Electricity } \\
\text { (cents/kWh) }\end{array}$ & Oil (cents/gal) & $\begin{array}{c}\text { Propane } \\
\text { (cents/gal) }\end{array}$ & $\begin{array}{c}\text { Gas }(\$ / 1000 \\
\left.\mathbf{f t}^{3}\right)\end{array}$ \\
\hline $\mathrm{AL}$ & 9.07 & 242 & 199 & 18.11 \\
\hline $\mathrm{AK}$ & 15.40 & 242 & 199 & 7.77 \\
\hline $\mathrm{AZ}$ & 9.76 & 242 & 199 & 16.75 \\
\hline $\mathrm{AR}$ & 8.95 & 242 & 199 & 12.47 \\
\hline $\mathrm{CA}$ & 14.90 & 242 & 199 & 10.96 \\
\hline $\mathrm{CO}$ & 9.12 & 242 & 199 & 8.95 \\
\hline $\mathrm{CT}$ & 17.25 & 249 & 221 & 15.91 \\
\hline $\mathrm{DE}$ & 12.47 & 244 & 246 & 16.32 \\
\hline $\mathrm{DC}$ & 10.37 & 273 & 236 & 15.52 \\
\hline FL & 11.33 & 231 & 215 & 20.02 \\
\hline GA & 9.37 & 231 & 215 & 16.26 \\
\hline $\mathrm{HI}$ & 24.00 & 242 & 199 & 33.66 \\
\hline ID & 6.29 & 242 & 199 & 11.92 \\
\hline IL & 9.00 & 231 & 179 & 9.80 \\
\hline $\mathrm{IN}$ & 8.58 & 230 & 189 & 10.84 \\
\hline IA & 10.02 & 226 & 150 & 11.45 \\
\hline $\mathrm{KS}$ & 8.68 & 231 & 179 & 12.12 \\
\hline KY & 7.39 & 220 & 202 & 11.13 \\
\hline LA & 9.16 & 242 & 199 & 13.19 \\
\hline $\mathrm{ME}$ & 15.13 & 227 & 243 & 16.54 \\
\hline MD & 10.29 & 243 & 252 & 14.62 \\
\hline MA & 16.90 & 233 & 228 & 16.21 \\
\hline MI & 10.34 & 235 & 193 & 10.53 \\
\hline $\mathrm{MN}$ & 8.94 & 232 & 171 & 10.78 \\
\hline MS & 9.67 & 242 & 199 & 12.45 \\
\hline $\mathrm{MO}$ & 8.10 & 231 & 166 & 13.09 \\
\hline MT & 8.46 & 242 & 199 & 9.58 \\
\hline $\mathrm{NE}$ & 8.10 & 215 & 144 & 10.49 \\
\hline NV & 11.04 & 242 & 199 & 14.08 \\
\hline $\mathrm{NH}$ & 14.98 & 238 & 238 & 15.82 \\
\hline $\mathrm{NJ}$ & 13.35 & 248 & 259 & 14.66 \\
\hline NM & 9.14 & 242 & 199 & 11.58 \\
\hline NY & 17.06 & 253 & 227 & 14.87 \\
\hline $\mathrm{NC}$ & 9.21 & 228 & 204 & 15.37 \\
\hline ND & 7.79 & 231 & 148 & 9.14 \\
\hline $\mathrm{OH}$ & 9.94 & 220 & 203 & 13.04 \\
\hline $\mathrm{OK}$ & 8.82 & 231 & 179 & 12.19 \\
\hline OR & 7.45 & 242 & 199 & 14.68 \\
\hline $\mathrm{PA}$ & 10.80 & 235 & 229 & 14.15 \\
\hline RI & 15.35 & 238 & 292 & 17.06 \\
\hline $\mathrm{SC}$ & 9.16 & 231 & 215 & 16.86 \\
\hline SD & 8.31 & 231 & 149 & 10.21 \\
\hline $\mathrm{TN}$ & 7.97 & 231 & 179 & 13.74 \\
\hline TX & 13.05 & 242 & 199 & 11.78 \\
\hline
\end{tabular}




\begin{tabular}{|c|c|c|c|c|}
\hline State & $\begin{array}{c}\text { Electricity } \\
(\text { cents/kWh) }\end{array}$ & Oil (cents/gal) & $\begin{array}{c}\text { Propane } \\
(\text { cents/gal) }\end{array}$ & $\begin{array}{c}\text { Gas (\$/1000 } \\
\mathbf{f t}^{\mathbf{3}} \mathbf{)}\end{array}$ \\
\hline UT & 7.86 & 242 & 199 & 9.65 \\
\hline VT & 13.71 & 256 & 249 & 15.22 \\
\hline VA & 8.86 & 232 & 230 & 14.82 \\
\hline WA & 6.77 & 234 & 199 & 13.88 \\
\hline WV & 6.49 & 231 & 215 & 15.00 \\
\hline WI & 10.64 & 231 & 178 & 11.43 \\
\hline WY & 8.20 & 242 & 199 & 9.29 \\
\hline VI & 24.00 & 242 & 199 & 33.66 \\
\hline PR & 24.00 & 242 & 199 & 33.66 \\
\hline GU & 24.00 & 242 & 199 & 33.66 \\
\hline
\end{tabular}


Table 12 Projected fuel price escalation indices (excluding general inflation) for Census Region 1 (Connecticut, Maine, Massachusetts, New Hampshire, New Jersey, New York, Pennsylvania, Rhode Island, Vermont)

\begin{tabular}{|l|c|c|c|c|}
\hline & Electricity & Distillate Oil & LPG & Natural Gas \\
\hline $\mathbf{2 0 0 8}$ & 0.98 & 0.99 & 1.03 & 0.97 \\
\hline $\mathbf{2 0 0 9}$ & 0.98 & 0.93 & 1.01 & 0.94 \\
\hline $\mathbf{2 0 1 0}$ & 0.97 & 0.87 & 0.99 & 0.91 \\
\hline $\mathbf{2 0 1 1}$ & 0.96 & 0.82 & 0.98 & 0.89 \\
\hline $\mathbf{2 0 1 2}$ & 0.96 & 0.79 & 0.97 & 0.87 \\
\hline $\mathbf{2 0 1 3}$ & 0.97 & 0.74 & 0.96 & 0.86 \\
\hline $\mathbf{2 0 1 4}$ & 0.96 & 0.73 & 0.96 & 0.85 \\
\hline $\mathbf{2 0 1 5}$ & 0.96 & 0.73 & 0.96 & 0.85 \\
\hline $\mathbf{2 0 1 6}$ & 0.97 & 0.73 & 0.96 & 0.85 \\
\hline $\mathbf{2 0 1 7}$ & 0.97 & 0.74 & 0.96 & 0.87 \\
\hline $\mathbf{2 0 1 8}$ & 0.98 & 0.74 & 0.96 & 0.87 \\
\hline $\mathbf{2 0 1 9}$ & 0.98 & 0.75 & 0.96 & 0.87 \\
\hline $\mathbf{2 0 2 0}$ & 0.98 & 0.76 & 0.96 & 0.88 \\
\hline $\mathbf{2 0 2 1}$ & 0.98 & 0.77 & 0.96 & 0.87 \\
\hline $\mathbf{2 0 2 2}$ & 0.98 & 0.78 & 0.96 & 0.88 \\
\hline $\mathbf{2 0 2 3}$ & 0.98 & 0.78 & 0.97 & 0.89 \\
\hline $\mathbf{2 0 2 4}$ & 0.99 & 0.78 & 0.97 & 0.9 \\
\hline $\mathbf{2 0 2 5}$ & 0.99 & 0.79 & 0.97 & 0.9 \\
\hline $\mathbf{2 0 2 6}$ & 0.99 & 0.79 & 0.97 & 0.91 \\
\hline $\mathbf{2 0 2 7}$ & 0.99 & 0.8 & 0.98 & 0.91 \\
\hline $\mathbf{2 0 2 8}$ & 1 & 0.8 & 0.98 & 0.92 \\
\hline $\mathbf{2 0 2 9}$ & 1 & 0.81 & 0.98 & 0.93 \\
\hline $\mathbf{2 0 3 0}$ & 1 & 0.82 & 0.99 & 0.94 \\
\hline $\mathbf{2 0 3 1}$ & 1 & 0.82 & 0.99 & 0.94 \\
\hline $\mathbf{2 0 3 2}$ & 1.01 & 0.82 & 0.99 & 0.95 \\
\hline $\mathbf{2 0 3 3}$ & 1.01 & 0.82 & 0.99 & 0.95 \\
\hline $\mathbf{2 0 3 4}$ & 1.01 & 0.82 & 0.99 & 0.96 \\
\hline $\mathbf{2 0 3 5}$ & 1.01 & 0.82 & 0.99 & 0.97 \\
\hline $\mathbf{2 0 3 6}$ & 1.02 & 0.82 & 0.99 & 0.99 \\
\hline $\mathbf{2 0 3 7}$ & 1.02 & 0.82 & & \\
\hline & & & 0.98 & \\
\hline
\end{tabular}


Table 13 Projected fuel price escalation indices (excluding general inflation) for Census Region 2 (Illinois, Indiana, Iowa, Kansas, Michigan, Minnesota, Missouri, Nebraska, North Dakota, Ohio, South Dakota, Wisconsin)

\begin{tabular}{|l|c|c|c|c|}
\hline & Electricity & Distillate Oil & LPG & Natural Gas \\
\hline $\mathbf{2 0 0 8}$ & 1.01 & 0.98 & 1.05 & 0.97 \\
\hline $\mathbf{2 0 0 9}$ & 1.02 & 0.93 & 1.03 & 0.93 \\
\hline $\mathbf{2 0 1 0}$ & 1.02 & 0.86 & 1.01 & 0.9 \\
\hline $\mathbf{2 0 1 1}$ & 1.02 & 0.8 & 1 & 0.87 \\
\hline $\mathbf{2 0 1 2}$ & 1.01 & 0.74 & 0.99 & 0.85 \\
\hline $\mathbf{2 0 1 3}$ & 1.01 & 0.7 & 0.98 & 0.84 \\
\hline $\mathbf{2 0 1 4}$ & 1 & 0.68 & 0.98 & 0.84 \\
\hline $\mathbf{2 0 1 5}$ & 1 & 0.68 & 0.97 & 0.83 \\
\hline $\mathbf{2 0 1 6}$ & 1 & 0.68 & 0.98 & 0.84 \\
\hline $\mathbf{2 0 1 7}$ & 1.01 & 0.69 & 0.99 & 0.86 \\
\hline $\mathbf{2 0 1 9}$ & 1.02 & 0.7 & 0.99 & 0.86 \\
\hline $\mathbf{2 0 2 0}$ & 1.02 & 0.71 & 0.98 & 0.86 \\
\hline $\mathbf{2 0 2 1}$ & 1.02 & 0.71 & 0.99 & 0.86 \\
\hline $\mathbf{2 0 2 2}$ & 1.02 & 0.72 & 0.99 & 0.86 \\
\hline $\mathbf{2 0 2 3}$ & 1.02 & 0.73 & 0.99 & 0.87 \\
\hline $\mathbf{2 0 2 4}$ & 1.02 & 0.73 & 1 & 0.89 \\
\hline $\mathbf{2 0 2 5}$ & 1.02 & 0.73 & 1 & 0.9 \\
\hline $\mathbf{2 0 2 6}$ & 1.02 & 0.74 & 1 & 0.9 \\
\hline $\mathbf{2 0 2 7}$ & 1.02 & 0.74 & 1 & 0.9 \\
\hline $\mathbf{2 0 2 8}$ & 1.02 & 0.75 & 1.01 & 0.91 \\
\hline $\mathbf{2 0 2 9}$ & 1.02 & 0.75 & 1.01 & 0.92 \\
\hline $\mathbf{2 0 3 0}$ & 1.02 & 0.76 & 1.02 & 0.93 \\
\hline $\mathbf{2 0 3 1}$ & 1.02 & 0.77 & 1.02 & 0.94 \\
\hline $\mathbf{2 0 3 2}$ & 1.02 & 0.77 & 1.02 & 0.95 \\
\hline $\mathbf{2 0 3 3}$ & 1.02 & 0.77 & 1.02 & 0.95 \\
\hline $\mathbf{2 0 3 4}$ & 1.02 & 0.77 & 1.02 & 0.96 \\
\hline $\mathbf{2 0 3 5}$ & 1.02 & 0.77 & 1.02 & 0.97 \\
\hline $\mathbf{2 0 3 6}$ & 1.02 & 0.77 & 1.02 & 0.97 \\
\hline $\mathbf{2 0 3 7}$ & 1.02 & 0.77 & 1.03 & 1.03 \\
\hline & 1.02 & 0.77 & & \\
\hline
\end{tabular}


Table 14 Projected fuel price escalation indices (excluding general inflation) for Census Region 3 (Alabama, Arkansas, Delaware, District of Columbia, Florida, Georgia, Kentucky, Louisiana, Maryland, Mississippi, North Carolina, Oklahoma, South Carolina, Tennessee

\begin{tabular}{|l|c|c|c|c|}
\hline & Electricity & Distillate Oil & LPG & Natural Gas \\
\hline $\mathbf{2 0 0 8}$ & 0.99 & 0.99 & 1.03 & 0.97 \\
\hline $\mathbf{2 0 0 9}$ & 0.98 & 0.93 & 1.01 & 0.94 \\
\hline $\mathbf{2 0 1 0}$ & 0.97 & 0.88 & 1 & 0.91 \\
\hline $\mathbf{2 0 1 1}$ & 0.96 & 0.83 & 0.98 & 0.88 \\
\hline $\mathbf{2 0 1 2}$ & 0.94 & 0.79 & 0.97 & 0.86 \\
\hline $\mathbf{2 0 1 3}$ & 0.93 & 0.74 & 0.96 & 0.85 \\
\hline $\mathbf{2 0 1 4}$ & 0.93 & 0.73 & 0.96 & 0.85 \\
\hline $\mathbf{2 0 1 5}$ & 0.93 & 0.73 & 0.96 & 0.85 \\
\hline $\mathbf{2 0 1 6}$ & 0.93 & 0.73 & 0.96 & 0.86 \\
\hline $\mathbf{2 0 1 7}$ & 0.93 & 0.74 & 0.97 & 0.87 \\
\hline $\mathbf{2 0 1 8}$ & 0.94 & 0.75 & 0.97 & 0.88 \\
\hline $\mathbf{2 0 1 9}$ & 0.94 & 0.75 & 0.97 & 0.88 \\
\hline $\mathbf{2 0 2 0}$ & 0.94 & 0.76 & 0.97 & 0.89 \\
\hline $\mathbf{2 0 2 1}$ & 0.94 & 0.77 & 0.97 & 0.89 \\
\hline $\mathbf{2 0 2 2}$ & 0.94 & 0.78 & 0.98 & 0.89 \\
\hline $\mathbf{2 0 2 3}$ & 0.94 & 0.78 & 0.98 & 0.91 \\
\hline $\mathbf{2 0 2 4}$ & 0.94 & 0.78 & 0.99 & 0.92 \\
\hline $\mathbf{2 0 2 5}$ & 0.95 & 0.79 & 0.99 & 0.92 \\
\hline $\mathbf{2 0 2 6}$ & 0.95 & 0.79 & 0.99 & 0.92 \\
\hline $\mathbf{2 0 2 7}$ & 0.95 & 0.8 & 0.99 & 0.93 \\
\hline $\mathbf{2 0 2 8}$ & 0.95 & 0.81 & 0.99 & 0.94 \\
\hline $\mathbf{2 0 2 9}$ & 0.95 & 0.81 & 1 & 0.95 \\
\hline $\mathbf{2 0 3 0}$ & 0.95 & 0.82 & 1 & 0.95 \\
\hline $\mathbf{2 0 3 1}$ & 0.95 & 0.82 & 1 & 0.96 \\
\hline $\mathbf{2 0 3 2}$ & 0.95 & 0.82 & 1 & 0.96 \\
\hline $\mathbf{2 0 3 3}$ & 0.96 & 0.82 & 1 & 0.97 \\
\hline $\mathbf{2 0 3 4}$ & 0.96 & 0.82 & 1 & 0.98 \\
\hline $\mathbf{2 0 3 5}$ & 0.96 & 0.82 & 1 & 0.98 \\
\hline $\mathbf{2 0 3 6}$ & 0.96 & 0.82 & 1 & 1 \\
\hline $\mathbf{2 0 3 7}$ & 0.96 & 0.83 & 1 & \\
\hline & & & & \\
\hline
\end{tabular}


Table 15 Projected fuel price escalation indices (excluding general inflation) for Census Region 4 (Alaska, Arizona, California, Colorado, Hawaii, Idaho, Montana, Nevada, New Mexico, Oregon, Utah, Washington, Wyoming)

\begin{tabular}{|l|c|c|c|c|}
\hline & Electricity & Distillate Oil & LPG & Natural Gas \\
\hline $\mathbf{2 0 0 8}$ & 1 & 0.96 & 1.03 & 0.97 \\
\hline $\mathbf{2 0 0 9}$ & 0.99 & 0.92 & 1.02 & 0.95 \\
\hline $\mathbf{2 0 1 0}$ & 0.97 & 0.86 & 1 & 0.92 \\
\hline $\mathbf{2 0 1 1}$ & 0.95 & 0.81 & 0.99 & 0.89 \\
\hline $\mathbf{2 0 1 2}$ & 0.93 & 0.76 & 0.98 & 0.88 \\
\hline $\mathbf{2 0 1 3}$ & 0.93 & 0.71 & 0.97 & 0.86 \\
\hline $\mathbf{2 0 1 4}$ & 0.93 & 0.71 & 0.97 & 0.86 \\
\hline $\mathbf{2 0 1 5}$ & 0.93 & 0.7 & 0.97 & 0.86 \\
\hline $\mathbf{2 0 1 6}$ & 0.93 & 0.71 & 0.97 & 0.86 \\
\hline $\mathbf{2 0 1 7}$ & 0.94 & 0.71 & 0.98 & 0.88 \\
\hline $\mathbf{2 0 1 8}$ & 0.94 & 0.72 & 0.97 & 0.86 \\
\hline $\mathbf{2 0 1 9}$ & 0.94 & 0.74 & 0.96 & 0.85 \\
\hline $\mathbf{2 0 2 0}$ & 0.94 & 0.75 & 0.96 & 0.85 \\
\hline $\mathbf{2 0 2 1}$ & 0.94 & 0.76 & 0.97 & 0.86 \\
\hline $\mathbf{2 0 2 2}$ & 0.94 & 0.77 & 0.97 & 0.87 \\
\hline $\mathbf{2 0 2 3}$ & 0.95 & 0.77 & 0.98 & 0.89 \\
\hline $\mathbf{2 0 2 4}$ & 0.95 & 0.78 & 0.98 & 0.9 \\
\hline $\mathbf{2 0 2 5}$ & 0.95 & 0.79 & 0.99 & 0.9 \\
\hline $\mathbf{2 0 2 6}$ & 0.96 & 0.8 & 0.99 & 0.91 \\
\hline $\mathbf{2 0 2 7}$ & 0.96 & 0.81 & 0.99 & 0.91 \\
\hline $\mathbf{2 0 2 8}$ & 0.96 & 0.82 & 1 & 0.93 \\
\hline $\mathbf{2 0 2 9}$ & 0.96 & 0.83 & 1 & 0.94 \\
\hline $\mathbf{2 0 3 0}$ & 0.97 & 0.84 & 1 & 0.95 \\
\hline $\mathbf{2 0 3 1}$ & 0.97 & 0.84 & 1.01 & 0.96 \\
\hline $\mathbf{2 0 3 2}$ & 0.98 & 0.84 & 1.01 & 0.96 \\
\hline $\mathbf{2 0 3 3}$ & 0.98 & 0.84 & 1.01 & 0.97 \\
\hline $\mathbf{2 0 3 4}$ & 0.98 & 0.84 & 1.01 & 0.98 \\
\hline $\mathbf{2 0 3 5}$ & 0.99 & 0.84 & 1.01 & 0.99 \\
\hline $\mathbf{2 0 3 6}$ & 0.99 & 0.84 & 1.01 & 1 \\
\hline $\mathbf{2 0 3 7}$ & 1 & 0.84 & 1.01 & \\
\hline & & & & \\
\hline
\end{tabular}


Table 16 Zip codes, heating degree days (HDD, base $65, \div 1000$ ), cooling degree hours (CDH, base $74 \mathrm{~F}, \div 1000$ ), fuel price escalation region (FER), insulation cost factor (ICF), IECC region

\begin{tabular}{|c|c|c|c|c|c|c|c|}
\hline Zip & Location & State & HDD & CDH & FER & ICF & IECC \\
\hline 1 & ZIP NOT ASSIGNED & & 0 & 0 & 0 & & 0 \\
\hline 2 & ZIP NOT ASSIGNED & & 0 & 0 & 0 & & 0 \\
\hline 3 & ZIP NOT ASSIGNED & & 0 & 0 & 0 & & 0 \\
\hline 4 & ZIP NOT_ASSIGNED & & 0 & 0 & 0 & & 0 \\
\hline 5 & Holtzville & NY & 5.4 & 3.7 & 1 & 127 & 4 \\
\hline 6 & Puerto Rico & PR & 0 & 55.2 & 3 & 93 & 1 \\
\hline 7 & Puerto Rico & PR & 0 & 55.2 & 3 & 93 & 1 \\
\hline 8 & Virgin Islands & VI & 0 & 55.1 & 3 & 93 & 1 \\
\hline 9 & Puerto Rico & PR & 0 & 55.2 & 3 & 93 & 1 \\
\hline 10 & Amherst & MA & 6.9 & 2.9 & 1 & 104 & 5 \\
\hline 11 & Springfield & MA & 6.9 & 5.2 & 1 & 104 & 5 \\
\hline 12 & Pittsfield & MA & 7.4 & 1.2 & 1 & 103 & 5 \\
\hline 13 & Greenfield & MA & 6.9 & 2.9 & 1 & 103 & 5 \\
\hline 14 & Fitchburg & MA & 6.8 & 3.3 & 1 & 113 & 5 \\
\hline 15 & Worcester & MA & 6.8 & 1.5 & 1 & 113 & 5 \\
\hline 16 & Worcester & MA & 6.8 & 1.5 & 1 & 113 & 5 \\
\hline 17 & Framingham & MA & 6.1 & 4.2 & 1 & 115 & 5 \\
\hline 18 & Lawrence & MA & 6.5 & 3.6 & 1 & 114 & 5 \\
\hline 19 & Glouchester & MA & 5.7 & 1.1 & 1 & 115 & 5 \\
\hline 20 & Boston & MA & 5.6 & 5.4 & 1 & 117 & 5 \\
\hline 21 & Boston & MA & 5.6 & 5.4 & 1 & 117 & 5 \\
\hline 22 & Boston & MA & 5.6 & 5.4 & 1 & 117 & 5 \\
\hline 23 & Brockton & MA & 6 & 3.4 & 1 & 115 & 5 \\
\hline 24 & Wellesley & MA & 6 & 4.2 & 1 & 117 & 5 \\
\hline 25 & Buzzards Bay & MA & 5.7 & 0.9 & 1 & 114 & 5 \\
\hline 26 & Hyannis & MA & 6 & 2 & 1 & 114 & 5 \\
\hline 27 & New Bedford & MA & 5.7 & 6.4 & 1 & 114 & 5 \\
\hline 28 & Kingston & RI & 6 & 1.7 & 1 & 104 & 5 \\
\hline 29 & Providence & RI & 5.8 & 3.6 & 1 & 104 & 5 \\
\hline 30 & Nashua & $\mathrm{NH}$ & 6.8 & 1.6 & 1 & 95 & 5 \\
\hline 31 & Manchester & $\mathrm{NH}$ & 7.5 & 1.6 & 1 & 94 & 6 \\
\hline 32 & Plymouth & $\mathrm{NH}$ & 8.3 & 1.3 & 1 & 94 & 6 \\
\hline 33 & Concord & $\mathrm{NH}$ & 7.5 & 2 & 1 & 94 & 6 \\
\hline 34 & Keene & $\mathrm{NH}$ & 7.5 & 2.2 & 1 & 77 & 6 \\
\hline 35 & Bethlehem & $\mathrm{NH}$ & 8.3 & 1.2 & 1 & 78 & 6 \\
\hline 36 & Charlestown & $\mathrm{NH}$ & 7.5 & 2.2 & 1 & 76 & 6 \\
\hline 37 & Hanover & $\mathrm{NH}$ & 7.3 & 1.9 & 1 & 76 & 6 \\
\hline 38 & Durham & $\mathrm{NH}$ & 6.7 & 2.2 & 1 & 97 & 5 \\
\hline 39 & Kittery & $\mathrm{ME}$ & 7.6 & 2.2 & 1 & 81 & 6 \\
\hline 40 & Bridgton & $\mathrm{ME}$ & 8.2 & 1.1 & 1 & 83 & 6 \\
\hline 41 & Portland & $\mathrm{ME}$ & 7.3 & 1.1 & 1 & 83 & 6 \\
\hline 42 & Lewiston & $\mathrm{ME}$ & 7.1 & 2.3 & 1 & 82 & 6 \\
\hline
\end{tabular}




\begin{tabular}{|c|c|c|c|c|c|c|c|}
\hline Zip & Location & State & HDD & CDH & FER & ICF & IECC \\
\hline 43 & Augusta & $\mathrm{ME}$ & 7.4 & 2.2 & 1 & 80 & 6 \\
\hline 44 & Bangor & ME & 7.7 & 1.2 & 1 & 82 & 6 \\
\hline 45 & Bath & $\mathrm{ME}$ & 7.3 & 1.1 & 1 & 82 & 6 \\
\hline 46 & Ellsworth & $\mathrm{ME}$ & 7.5 & 1 & 1 & 82 & 6 \\
\hline 47 & Caribou & $\mathrm{ME}$ & 9.6 & 0.9 & 1 & 82 & 6 \\
\hline 48 & Rockland & $\mathrm{ME}$ & 7.4 & 1 & 1 & 82 & 6 \\
\hline 49 & Waterville & $\mathrm{ME}$ & 8 & 1.8 & 1 & 81 & 6 \\
\hline 50 & Woodstock & VT & 8.1 & 1.1 & 1 & 74 & 6 \\
\hline 51 & Bellows Falls & VT & 7.2 & 2.2 & 1 & 80 & 6 \\
\hline 52 & Bennington & VT & 7.9 & 0.8 & 1 & 81 & 6 \\
\hline 53 & Brattleboro & VT & 7.2 & 2.2 & 1 & 81 & 6 \\
\hline 54 & Burlington & VT & 7.7 & 2.6 & 1 & 80 & 6 \\
\hline 55 & ZIP NOT ASSIGNED & & 0 & 0 & 0 & 0 & 0 \\
\hline 56 & Montpelier & VT & 8.2 & 1.4 & 1 & 80 & 6 \\
\hline 57 & Rutland & VT & 7.3 & 1.8 & 1 & 80 & 6 \\
\hline 58 & St. Johnsbury & VT & 7.8 & 1.9 & 1 & 76 & 6 \\
\hline 59 & Canaan & VT & 8.9 & 1.5 & 1 & 76 & 6 \\
\hline 60 & Hartford & $\mathrm{CT}$ & 6.1 & 4.8 & 1 & 108 & 5 \\
\hline 61 & Hartford & $\mathrm{CT}$ & 6.1 & 4.8 & 1 & 108 & 5 \\
\hline 62 & Storrs & $\mathrm{CT}$ & 6.4 & 1.3 & 1 & 107 & 5 \\
\hline 63 & Norwich & $\mathrm{CT}$ & 5.9 & 1.7 & 1 & 107 & 5 \\
\hline 64 & Milford & $\mathrm{CT}$ & 5.4 & 5 & 1 & 107 & 5 \\
\hline 65 & New Haven & $\mathrm{CT}$ & 5.4 & 5 & 1 & 108 & 5 \\
\hline 66 & Bridgeport & $\mathrm{CT}$ & 5.4 & 5 & 1 & 109 & 5 \\
\hline 67 & Waterbury & $\mathrm{CT}$ & 6.1 & 2 & 1 & 108 & 5 \\
\hline 68 & Danbury & $\mathrm{CT}$ & 6.1 & 2.9 & 1 & 110 & 5 \\
\hline 69 & Stamford & $\mathrm{CT}$ & 5.6 & 3.6 & 1 & 110 & 5 \\
\hline 70 & Boonton & NJ & 5.8 & 3.9 & 1 & 111 & 5 \\
\hline 71 & Newark & NJ & 4.8 & 9.1 & 1 & 111 & 4 \\
\hline 72 & Elizabeth & $\mathrm{NJ}$ & 4.8 & 9.1 & 1 & 111 & 4 \\
\hline 73 & Jersey City & NJ & 5.4 & 7 & 1 & 110 & 5 \\
\hline 74 & West Milford & $\mathrm{NJ}$ & 6.4 & 3.9 & 1 & 108 & 5 \\
\hline 75 & Paterson & NJ & 4.8 & 6.1 & 1 & 108 & 4 \\
\hline 76 & Hackensack & $\mathrm{NJ}$ & 4.8 & 6.1 & 1 & 108 & 4 \\
\hline 77 & Long Branch & NJ & 5.2 & 5.2 & 1 & 111 & 4 \\
\hline 78 & Newton & $\mathrm{NJ}$ & 6.4 & 2.8 & 1 & 111 & 5 \\
\hline 79 & Morris Plains & $\mathrm{NJ}$ & 5.9 & 4 & 1 & 111 & 5 \\
\hline 80 & Hammonton & $\mathrm{NJ}$ & 5 & 7.6 & 1 & 108 & 4 \\
\hline 81 & Camden & $\mathrm{NJ}$ & 4.8 & 8.9 & 1 & 108 & 4 \\
\hline 82 & Atlantic City & NJ & 5.1 & 6.2 & 1 & 108 & 4 \\
\hline 83 & Millville & NJ & 4.8 & 7.4 & 1 & 108 & 4 \\
\hline 84 & Atlantic City & NJ & 5.1 & 6.2 & 1 & 108 & 4 \\
\hline 85 & Hightstown & $\mathrm{NJ}$ & 5.4 & 5.5 & 1 & 107 & 5 \\
\hline 86 & Trenton & $\mathrm{NJ}$ & 5.4 & 7.4 & 1 & 107 & 5 \\
\hline 87 & Toms River & $\mathrm{NJ}$ & 5.2 & 7.6 & 1 & 111 & 4 \\
\hline 88 & Somerville & NJ & 5.8 & 5.7 & 1 & 110 & 4 \\
\hline 89 & New Brunswick & NJ & 5.3 & 5.7 & 1 & 110 & 4 \\
\hline 90 & ZIP NOT ASSIGNED & & 0 & 0 & 0 & 0 & 0 \\
\hline
\end{tabular}




\begin{tabular}{|c|c|c|c|c|c|c|c|}
\hline Zip & Location & State & HDD & CDH & FER & ICF & IECC \\
\hline 91 & ZIP_NOT_ASSIGNED & & 0 & 0 & 0 & 0 & 0 \\
\hline 92 & ZIP_NOT_ASSIGNED & & 0 & 0 & 0 & 0 & 0 \\
\hline 93 & ZIP NOT ASSIGNED & & 0 & 0 & 0 & 0 & 0 \\
\hline 94 & ZIP_NOT_ASSIGNED & & 0 & 0 & 0 & 0 & 0 \\
\hline 95 & ZIP_NOT_ASSIGNED & & 0 & 0 & 0 & 0 & 0 \\
\hline 96 & ZIP_NOT_ASSIGNED & & 0 & 0 & 0 & 0 & 0 \\
\hline 97 & ZIP_NOT_ASSIGNED & & 0 & 0 & 0 & 0 & 0 \\
\hline 98 & ZIP_NOT_ASSIGNED & & 0 & 0 & 0 & 0 & 0 \\
\hline 99 & ZIP_NOT_ASSIGNED & & 0 & 0 & 0 & 0 & 0 \\
\hline 100 & New York & NY & 4.9 & 9.5 & 1 & 132 & 4 \\
\hline 101 & New York & NY & 4.9 & 9.5 & 1 & 132 & 4 \\
\hline 102 & New York & NY & 4.9 & 9.5 & 1 & 132 & 4 \\
\hline 103 & Staten Island & NY & 4.9 & 7 & 1 & 128 & 4 \\
\hline 104 & Bronx & NY & 4.9 & 9.2 & 1 & 128 & 4 \\
\hline 105 & Scarsdale & NY & 5.4 & 5.4 & 1 & 122 & 4 \\
\hline 106 & White Plains & NY & 5.4 & 5.4 & 1 & 122 & 4 \\
\hline 107 & Yonkers & NY & 5.4 & 5.4 & 1 & 122 & 4 \\
\hline 108 & New Rochelle & NY & 5.4 & 5.4 & 1 & 122 & 4 \\
\hline 109 & Suffern & NY & 5.8 & 6.1 & 1 & 120 & 4 \\
\hline 110 & Great Neck & NY & 4.9 & 7.6 & 1 & 127 & 4 \\
\hline 111 & Woodside & NY & 4.9 & 7.6 & 1 & 127 & 4 \\
\hline 112 & Brooklyn & NY & 4.9 & 7.6 & 1 & 127 & 4 \\
\hline 113 & Flushing & NY & 4.9 & 7.6 & 1 & 127 & 4 \\
\hline 114 & Queens & NY & 4.9 & 7.6 & 1 & 127 & 4 \\
\hline 115 & Minneola & NY & 5.2 & 7.4 & 1 & 124 & 4 \\
\hline 116 & Far Rockaway & NY & 4.9 & 7.6 & 1 & 127 & 4 \\
\hline 117 & Mid-Island & NY & 4.9 & 7.6 & 1 & 124 & 4 \\
\hline 118 & Hicksville & NY & 4.9 & 7.6 & 1 & 124 & 4 \\
\hline 119 & Riverhead & NY & 5.1 & 4.7 & 1 & 124 & 4 \\
\hline 120 & Gloversville & NY & 7.6 & 1.8 & 1 & 92 & 6 \\
\hline 121 & Troy & NY & 6.6 & 3 & 1 & 92 & 5 \\
\hline 122 & Albany & NY & 6.9 & 3 & 1 & 92 & 5 \\
\hline 123 & Schenectady & NY & 6.6 & 3 & 1 & 92 & 5 \\
\hline 124 & Cairo & NY & 6.9 & 1.8 & 1 & 119 & 5 \\
\hline 125 & Glenham & NY & 5.8 & 6 & 1 & 119 & 5 \\
\hline 126 & Poughkeepsie & NY & 6.4 & 4.3 & 1 & 119 & 5 \\
\hline 127 & Liberty & NY & 7.5 & 0.8 & 1 & 119 & 6 \\
\hline 128 & Saratoga Springs & NY & 6.9 & 1.8 & 1 & 91 & 5 \\
\hline 129 & Plattsburgh & NY & 7.8 & 1.9 & 1 & 94 & 6 \\
\hline 130 & Syracuse & NY & 6.8 & 3.5 & 1 & 99 & 5 \\
\hline 131 & Syracuse & NY & 6.8 & 3.5 & 1 & 99 & 5 \\
\hline 132 & Syracuse & NY & 6.8 & 3.5 & 1 & 99 & 5 \\
\hline 133 & Utica & NY & 7.2 & 2.7 & 1 & 92 & 6 \\
\hline 134 & Old Forge & NY & 9 & 2.7 & 1 & 92 & 6 \\
\hline 135 & Utica & NY & 7.2 & 2.7 & 1 & 92 & 6 \\
\hline 136 & Watertown & NY & 7.7 & 2.7 & 1 & 91 & 6 \\
\hline 137 & Norwich & NY & 7.1 & 1.3 & 1 & 96 & 5 \\
\hline 138 & Binghamton & NY & 7.2 & 1.6 & 1 & 96 & 6 \\
\hline
\end{tabular}




\begin{tabular}{|c|c|c|c|c|c|c|c|}
\hline Zip & Location & State & HDD & CDH & FER & ICF & IECC \\
\hline 139 & Binghamton & NY & 7.2 & 1.6 & 1 & 96 & 6 \\
\hline 140 & Buffalo & NY & 6.7 & 3 & 1 & 104 & 5 \\
\hline 141 & Buffalo & NY & 6.7 & 3 & 1 & 104 & 5 \\
\hline 142 & Buffalo & NY & 6.7 & 3 & 1 & 104 & 5 \\
\hline 143 & Niagra Falls & NY & 6.7 & 3 & 1 & 102 & 5 \\
\hline 144 & Rochester & NY & 6.7 & 3.8 & 1 & 96 & 5 \\
\hline 145 & Rochester & NY & 6.7 & 3.8 & 1 & 96 & 5 \\
\hline 146 & Rochester & $\mathrm{NY}$ & 6.7 & 3.8 & 1 & 96 & 5 \\
\hline 147 & Jamestown & NY & 7 & 3.7 & 1 & 96 & 5 \\
\hline 148 & Ithaca & NY & 7.2 & 1.6 & 1 & 90 & 6 \\
\hline 149 & Elmira & NY & 6.8 & 2.5 & 1 & 90 & 5 \\
\hline 150 & Donora & PA & 5.5 & 8.4 & 1 & 102 & 5 \\
\hline 151 & Pittsburgh & PA & 5.8 & 5 & 1 & 102 & 5 \\
\hline 152 & Pittsburgh & $\mathrm{PA}$ & 5.8 & 5 & 1 & 102 & 5 \\
\hline 153 & Washington & PA & 6.3 & 3.9 & 1 & 101 & 5 \\
\hline 154 & Uniontown & PA & 5.7 & 6.2 & 1 & 100 & 5 \\
\hline 155 & Everett & PA & 6.2 & 6.1 & 1 & 97 & 5 \\
\hline 156 & Derry & PA & 5.9 & 8.4 & 1 & 101 & 5 \\
\hline 157 & Indiana & PA & 6 & 2.6 & 1 & 100 & 5 \\
\hline 158 & Du Bois & PA & 6.8 & 1.8 & 1 & 100 & 5 \\
\hline 159 & Johnstown & PA & 5.5 & 5.7 & 1 & 99 & 5 \\
\hline 160 & Butler & PA & 6.6 & 4.7 & 1 & 100 & 5 \\
\hline 161 & New Castle & $\mathrm{PA}$ & 6.2 & 4.8 & 1 & 99 & 5 \\
\hline 162 & Clarion & PA & 6.9 & 2.5 & 1 & 100 & 5 \\
\hline 163 & Franklin & PA & 6.5 & 2.8 & 1 & 98 & 5 \\
\hline 164 & Corry & PA & 6.8 & 2 & 1 & 99 & 5 \\
\hline 165 & Erie & PA & 6.2 & 2.2 & 1 & 99 & 5 \\
\hline 166 & Altoona & PA & 6 & 1.5 & 1 & 97 & 5 \\
\hline 167 & Bradford & PA & 7.7 & 0.8 & 1 & 98 & 6 \\
\hline 168 & State_College & PA & 6.3 & 3.5 & 1 & 98 & 5 \\
\hline 169 & Wellsboro & PA & 7.5 & 1.4 & 1 & 97 & 6 \\
\hline 170 & Lewiston & PA & 5.9 & 5.3 & 1 & 105 & 5 \\
\hline 171 & Harrisburg & PA & 5.2 & 9.1 & 1 & 105 & 4 \\
\hline 172 & Chambersburg & PA & 5.6 & 5.4 & 1 & 91 & 5 \\
\hline 173 & Hanover & PA & 5.3 & 7.2 & 1 & 102 & 4 \\
\hline 174 & York & PA & 5.2 & 6.6 & 1 & 102 & 4 \\
\hline 175 & Lancaster & PA & 5.4 & 6 & 1 & 100 & 5 \\
\hline 176 & Lancaster & $\mathrm{PA}$ & 5.4 & 6 & 1 & 100 & 5 \\
\hline 177 & Williamsport & PA & 6.1 & 5 & 1 & 98 & 5 \\
\hline 178 & Selinsgrove & PA & 6.1 & 5 & 1 & 101 & 5 \\
\hline 179 & Pottsville & PA & 5.4 & 3 & 1 & 101 & 5 \\
\hline 180 & Palmerton & PA & 5.5 & 5.5 & 1 & 103 & 5 \\
\hline 181 & Allentown & PA & 5.8 & 5.8 & 1 & 108 & 5 \\
\hline 182 & Freeland & PA & 7.5 & 1.7 & 1 & 101 & 6 \\
\hline 183 & Stroudsburg & PA & 6.2 & 5.3 & 1 & 94 & 5 \\
\hline 184 & Hawley & PA & 7 & 0.6 & 1 & 101 & 5 \\
\hline 185 & Scranton & PA & 6.2 & 3.8 & 1 & 101 & 5 \\
\hline 186 & Wilkes-Barre & PA & 6.2 & 3.8 & 1 & 102 & 5 \\
\hline
\end{tabular}




\begin{tabular}{|c|c|c|c|c|c|c|c|}
\hline Zip & Location & State & HDD & CDH & FER & ICF & IECC \\
\hline 187 & Wilkes-Barre & PA & 6.2 & 3.8 & 1 & 102 & 5 \\
\hline 188 & Montrose & PA & 7.4 & 1.6 & 1 & 99 & 6 \\
\hline 189 & Doylestown & PA & 5.4 & 6.5 & 1 & 110 & 5 \\
\hline 190 & Philadelphia & PA & 4.8 & 8.9 & 1 & 116 & 4 \\
\hline 191 & Philadelphia & PA & 4.8 & 8.9 & 1 & 116 & 4 \\
\hline 192 & Philadelphia & PA & 4.8 & 8.9 & 1 & 116 & 4 \\
\hline 193 & Southeastern_PA & PA & 4.8 & 8.9 & 1 & 112 & 4 \\
\hline 194 & Southeastern_PA & PA & 5.4 & 8.9 & 1 & 113 & 5 \\
\hline 195 & Hamburg & PA & 5.8 & 4 & 1 & 105 & 5 \\
\hline 196 & Reading & PA & 5.4 & 4 & 1 & 105 & 5 \\
\hline 197 & Newark & $\mathrm{DE}$ & 4.7 & 7.6 & 3 & 102 & 4 \\
\hline 198 & Wilmington & $\mathrm{DE}$ & 4.9 & 8.2 & 3 & 102 & 4 \\
\hline 199 & Dover & $\mathrm{DE}$ & 4.2 & 9.3 & 3 & 102 & 4 \\
\hline 200 & Washington & $\mathrm{DC}$ & 4.1 & 12.4 & 3 & 98 & 4 \\
\hline 201 & Dulles & $\mathrm{DC}$ & 4.9 & 7.7 & 3 & 98 & 4 \\
\hline 202 & Washington & $\mathrm{DC}$ & 4.1 & 12.4 & 3 & 98 & 4 \\
\hline 203 & Washington & $\mathrm{DC}$ & 4.1 & 12.4 & 3 & 98 & 4 \\
\hline 204 & Washington & $\mathrm{DC}$ & 4.1 & 12.4 & 3 & 98 & 4 \\
\hline 205 & Washington & $\mathrm{DC}$ & 4.1 & 12.4 & 3 & 98 & 4 \\
\hline 206 & LaPlata & MD & 4.2 & 8.2 & 3 & 94 & 4 \\
\hline 207 & Laurel & MD & 4.5 & 10.5 & 3 & 94 & 4 \\
\hline 208 & Rockville & MD & 5 & 9.8 & 3 & 94 & 4 \\
\hline 209 & Silver Spring & MD & 4.5 & 9.8 & 3 & 96 & 4 \\
\hline 210 & Baltimore & MD & 4.7 & 9.5 & 3 & 91 & 4 \\
\hline 211 & Baltimore & MD & 4.7 & 9.5 & 3 & 91 & 4 \\
\hline 212 & Baltimore & MD & 4.7 & 9.5 & 3 & 91 & 4 \\
\hline 213 & Not used & $\mathrm{NU}$ & 0 & 0 & 0 & 0 & 0 \\
\hline 214 & Annapolis & MD & 4.7 & 9.5 & 3 & 90 & 4 \\
\hline 215 & Cumberland & MD & 5.2 & 7.1 & 3 & 90 & 4 \\
\hline 216 & Centreville & MD & 4.4 & 9.1 & 3 & 75 & 4 \\
\hline 217 & Frederick & MD & 4.4 & 7.3 & 3 & 86 & 4 \\
\hline 218 & Salisbury & MD & 4.3 & 9.2 & 3 & 82 & 4 \\
\hline 219 & Elkton & MD & 4.6 & 6.6 & 3 & 78 & 4 \\
\hline 220 & Northern Virginia & VA & 4.1 & 12.4 & 3 & 93 & 4 \\
\hline 221 & Northern Virginia & VA & 4.1 & 12.4 & 3 & 93 & 4 \\
\hline 222 & Arlington & VA & 4.1 & 12.4 & 3 & 93 & 4 \\
\hline 223 & Alexandria & VA & 4.1 & 12.4 & 3 & 94 & 4 \\
\hline 224 & Fredericksburg & VA & 4.5 & 10.2 & 3 & 86 & 4 \\
\hline 225 & Colonial Beach & VA & 3.8 & 10.2 & 3 & 86 & 4 \\
\hline 226 & Winchester & VA & 5.1 & 8.1 & 3 & 89 & 4 \\
\hline 227 & Culpeper & VA & 4.2 & 8.9 & 3 & 91 & 4 \\
\hline 228 & Harrisonburg & VA & 5.4 & 6.5 & 3 & 91 & 4 \\
\hline 229 & Charlottesville & VA & 4.1 & 10.3 & 3 & 87 & 4 \\
\hline 230 & Amelia & VA & 4.4 & 11 & 3 & 88 & 4 \\
\hline 231 & Williamsburg & VA & 3.6 & 11 & 3 & 88 & 4 \\
\hline 232 & Richmond & VA & 3.9 & 12.3 & 3 & 88 & 4 \\
\hline 233 & Wallops Island & VA & 4.2 & 13.7 & 3 & 88 & 4 \\
\hline 234 & Wallops Island & VA & 4.2 & 13.7 & 3 & 88 & 4 \\
\hline
\end{tabular}




\begin{tabular}{|c|c|c|c|c|c|c|c|}
\hline Zip & Location & State & HDD & CDH & FER & ICF & IECC \\
\hline 235 & Norfolk & VA & 3.4 & 13.7 & 3 & 88 & 4 \\
\hline 236 & Norfolk & VA & 3.4 & 13.7 & 3 & 88 & 4 \\
\hline 237 & Portsmouth & VA & 3.4 & 13.7 & 3 & 87 & 4 \\
\hline 238 & Emporia & VA & 3.8 & 11.5 & 3 & 89 & 4 \\
\hline 239 & Farmville & VA & 4 & 9.4 & 3 & 85 & 4 \\
\hline 240 & Roanoke & VA & 4.3 & 9.3 & 3 & 88 & 4 \\
\hline 241 & Blacksburg & VA & 5.6 & 4.5 & 3 & 88 & 4 \\
\hline 242 & Abingdon & VA & 4.9 & 8.8 & 3 & 85 & 4 \\
\hline 243 & Pulaski & VA & 5.3 & 3.3 & 3 & 85 & 4 \\
\hline 244 & Staunton & VA & 5.4 & 6.5 & 3 & 87 & 4 \\
\hline 245 & Lynchburg & VA & 4.4 & 8.4 & 3 & 89 & 4 \\
\hline 246 & Richlands & VA & 5.3 & 2.6 & 3 & 85 & 4 \\
\hline 247 & Bluefield & WV & 4.9 & 2.6 & 3 & 96 & 4 \\
\hline 248 & Pineville & WV & 5 & 7.9 & 3 & 96 & 4 \\
\hline 249 & Lewisburg & WV & 5.8 & 3.7 & 3 & 98 & 4 \\
\hline 250 & Charleston & WV & 4.6 & 8.8 & 3 & 98 & 4 \\
\hline 251 & Charleston & WV & 4.6 & 8.8 & 3 & 98 & 4 \\
\hline 252 & Charleston & WV & 4.6 & 8.8 & 3 & 98 & 4 \\
\hline 253 & Charleston & WV & 4.6 & 8.8 & 3 & 98 & 4 \\
\hline 254 & Martinsburg & WV & 4.8 & 8.2 & 3 & 90 & 4 \\
\hline 255 & Dunlow & WV & 5 & 11.2 & 3 & 100 & 4 \\
\hline 256 & Logan & WV & 4.5 & 7.5 & 3 & 100 & 4 \\
\hline 257 & Huntington & WV & 4.7 & 11.2 & 3 & 100 & 4 \\
\hline 258 & Beckley & WV & 5.4 & 2.1 & 3 & 98 & 5 \\
\hline 259 & Oak Hill & WV & 5.4 & 4.1 & 3 & 98 & 5 \\
\hline 260 & Wheeling & WV & 5.3 & 6.8 & 3 & 97 & 4 \\
\hline 261 & Parkersburg & WV & 5.1 & 9.1 & 3 & 97 & 4 \\
\hline 262 & Buckhannon & WV & 5.7 & 4 & 3 & 97 & 5 \\
\hline 263 & Clarksburg & WV & 5.5 & 6.4 & 3 & 97 & 5 \\
\hline 264 & Clarksburg & WV & 5.5 & 6.4 & 3 & 97 & 5 \\
\hline 265 & Morgantown & WV & 5.3 & 6.9 & 3 & 97 & 4 \\
\hline 266 & Gassaway & WV & 5 & 6.3 & 3 & 97 & 4 \\
\hline 267 & Bayard & WV & 7 & 0.9 & 3 & 92 & 5 \\
\hline 268 & Moorefield & WV & 5.3 & 4 & 3 & 93 & 4 \\
\hline 269 & ZIP NOT ASSIGNED & & 0 & 0 & 0 & 0 & 0 \\
\hline 270 & Mt. Airy & $\mathrm{NC}$ & 4.5 & 8 & 3 & 82 & 4 \\
\hline 271 & Winston Salem & $\mathrm{NC}$ & 3.8 & 11.8 & 3 & 81 & 4 \\
\hline 272 & Burlington & $\mathrm{NC}$ & 3.6 & 13.6 & 3 & 82 & 3 \\
\hline 273 & Reidsville & $\mathrm{NC}$ & 3.9 & 12.9 & 3 & 82 & 4 \\
\hline 274 & Greensboro & $\mathrm{NC}$ & 3.8 & 11 & 3 & 82 & 4 \\
\hline 275 & Raleigh & $\mathrm{NC}$ & 3.5 & 11.8 & 3 & 82 & 3 \\
\hline 276 & Raleigh & $\mathrm{NC}$ & 3.5 & 11.8 & 3 & 82 & 3 \\
\hline 277 & Durham & $\mathrm{NC}$ & 3.7 & 11.8 & 3 & 83 & 4 \\
\hline 278 & Rocky Mount & $\mathrm{NC}$ & 3.3 & 13.8 & 3 & 75 & 3 \\
\hline 279 & Elizabeth City & $\mathrm{NC}$ & 3 & 14 & 3 & 76 & 3 \\
\hline 280 & Gastonia & $\mathrm{NC}$ & 3.4 & 15.9 & 3 & 82 & 3 \\
\hline 281 & Salisbury & $\mathrm{NC}$ & 3.9 & 13.4 & 3 & 82 & 4 \\
\hline 282 & Charlotte & $\mathrm{NC}$ & 3.2 & 15.2 & 3 & 82 & 3 \\
\hline
\end{tabular}




\begin{tabular}{|c|c|c|c|c|c|c|c|}
\hline Zip & Location & State & HDD & CDH & FER & ICF & IECC \\
\hline 283 & Fayetteville & $\mathrm{NC}$ & 3.1 & 15.6 & 3 & 82 & 3 \\
\hline 284 & Wilmington & $\mathrm{NC}$ & 2.4 & 17.6 & 3 & 81 & 3 \\
\hline 285 & Kinston & $\mathrm{NC}$ & 3.2 & 15.7 & 3 & 76 & 3 \\
\hline 286 & Hickory & $\mathrm{NC}$ & 3.6 & 11.1 & 3 & 81 & 3 \\
\hline 287 & Cullowhee & $\mathrm{NC}$ & 4.3 & 4.5 & 3 & 81 & 4 \\
\hline 288 & Asheville & $\mathrm{NC}$ & 4.3 & 6.2 & 3 & 81 & 4 \\
\hline 289 & Andrews & $\mathrm{NC}$ & 4.2 & 5.2 & 3 & 76 & 4 \\
\hline 290 & Columbia & $\mathrm{SC}$ & 2.6 & 22 & 3 & 79 & 3 \\
\hline 291 & Columbia & $\mathrm{SC}$ & 2.6 & 22 & 3 & 79 & 3 \\
\hline 292 & Columbia & $\mathrm{SC}$ & 2.6 & 22 & 3 & 79 & 3 \\
\hline 293 & Spartanburg & $\mathrm{SC}$ & 3.1 & 14.1 & 3 & 80 & 3 \\
\hline 294 & Charleston & $\mathrm{SC}$ & 2 & 23.3 & 3 & 80 & 3 \\
\hline 295 & Florence & $\mathrm{SC}$ & 2.5 & 17.9 & 3 & 80 & 3 \\
\hline 296 & Greenville & $\mathrm{SC}$ & 3.3 & 14.1 & 3 & 80 & 3 \\
\hline 297 & Chester & $\mathrm{SC}$ & 3.3 & 15.8 & 3 & 74 & 3 \\
\hline 298 & Aiken & $\mathrm{SC}$ & 2.4 & 20.3 & 3 & 91 & 3 \\
\hline 299 & Beaufort & $\mathrm{SC}$ & 2 & 21.5 & 3 & 76 & 3 \\
\hline 300 & Covington & GA & 3 & 16.9 & 3 & 92 & 3 \\
\hline 301 & Rome & GA & 3.5 & 17.7 & 3 & 92 & 3 \\
\hline 302 & Newnan & GA & 3.1 & 16.3 & 3 & 92 & 3 \\
\hline 303 & Atlanta & GA & 2.8 & 16.8 & 3 & 92 & 3 \\
\hline 304 & Swainsboro & GA & 2.2 & 23 & 3 & 78 & 3 \\
\hline 305 & Gainesville & GA & 3.1 & 14.8 & 3 & 81 & 4 \\
\hline 306 & Athens & GA & 2.9 & 16.1 & 3 & 84 & 3 \\
\hline 307 & Dalton & GA & 3.4 & 14.7 & 3 & 80 & 4 \\
\hline 308 & Augusta & GA & 2.5 & 19.5 & 3 & 83 & 3 \\
\hline 309 & Augusta & GA & 2.5 & 19.5 & 3 & 83 & 3 \\
\hline 310 & Macon & GA & 2.4 & 24.4 & 3 & 83 & 3 \\
\hline 311 & Atlanta & GA & 2.8 & 16.8 & 3 & 83 & 3 \\
\hline 312 & Macon & GA & 2.4 & 24.4 & 3 & 83 & 3 \\
\hline 313 & Ft. Stewart & GA & 1.6 & 23.7 & 3 & 82 & 2 \\
\hline 314 & Savannah & GA & 1.8 & 22.8 & 3 & 82 & 2 \\
\hline 315 & Waycross & GA & 1.7 & 23.6 & 3 & 77 & 2 \\
\hline 316 & Quitman & GA & 1.8 & 26.5 & 3 & 83 & 2 \\
\hline 317 & Albany & GA & 2.1 & 26.5 & 3 & 83 & 2 \\
\hline 318 & Columbus & GA & 2.2 & 22.1 & 3 & 87 & 3 \\
\hline 319 & Columbus & GA & 2.2 & 22.1 & 3 & 87 & 3 \\
\hline 320 & Jacksonville & FL & 1.4 & 24.2 & 3 & 82 & 2 \\
\hline 321 & Daytona Beach & FL & 0.8 & 27.1 & 3 & 86 & 2 \\
\hline 322 & Jacksonville & FL & 1.4 & 24.2 & 3 & 82 & 2 \\
\hline 323 & Tallahassee & FL & 1.6 & 25.2 & 3 & 80 & 2 \\
\hline 324 & Panama City & FL & 1.8 & 29 & 3 & 72 & 2 \\
\hline 325 & Pensacola & FL & 1.5 & 29 & 3 & 80 & 2 \\
\hline 326 & Gainesville & FL & 1.1 & 27.7 & 3 & 81 & 2 \\
\hline 327 & Titusville & FL & 0.7 & 29.9 & 3 & 86 & 2 \\
\hline 328 & Orlando & FL & 0.6 & 34 & 3 & 86 & 2 \\
\hline 329 & Melbourne & FL & 0.6 & 29.7 & 3 & 89 & 2 \\
\hline 330 & Miami & $\mathrm{FL}$ & 0.1 & 39 & 3 & 92 & 1 \\
\hline
\end{tabular}




\begin{tabular}{|c|c|c|c|c|c|c|c|}
\hline Zip & Location & State & HDD & $\mathrm{CDH}$ & FER & ICF & IECC \\
\hline 331 & Miami & $\mathrm{FL}$ & 0.1 & 39 & 3 & 92 & 1 \\
\hline 332 & Miami & FL & 0.1 & 39 & 3 & 92 & 1 \\
\hline 333 & Fort Lauderdale & FL & 0.2 & 37.1 & 3 & 88 & 1 \\
\hline 334 & West Palm Beach & FL & 0.2 & 35.2 & 3 & 85 & 1 \\
\hline 335 & Tampa & FL & 0.6 & 33.7 & 3 & 85 & 2 \\
\hline 336 & Tampa & FL & 0.6 & 33.7 & 3 & 85 & 2 \\
\hline 337 & Saint Petersburg & FL & 0.5 & 38.6 & 3 & 78 & 2 \\
\hline 338 & Lakeland & FL & 0.5 & 34.9 & 3 & 85 & 2 \\
\hline 339 & Fort Myers & FL & 0.3 & 37.4 & 3 & 83 & 2 \\
\hline 340 & Not used & NU & 0 & 0 & 0 & 0 & 0 \\
\hline 341 & Naples & FL & 0.3 & 34.4 & 3 & 83 & 1 \\
\hline 342 & Bradenton & FL & 0.6 & 29.2 & 3 & 85 & 2 \\
\hline 343 & ZIP_NOT_ASSIGNED & & 0 & 0 & 0 & 0 & 0 \\
\hline 344 & Ocala & FL & 0.9 & 32.2 & 3 & 81 & 2 \\
\hline 345 & ZIP_NOT_ASSIGNED & & 0 & 0 & 0 & 0 & 0 \\
\hline 346 & Brooksville & FL & 0.7 & 29.7 & 3 & 85 & 2 \\
\hline 347 & Kissimmee & FL & 0.7 & 34 & 3 & 86 & 2 \\
\hline 348 & ZIP_NOT_ASSIGNED & & 0 & 0 & 0 & 0 & 0 \\
\hline 349 & Fort Pierce & FL & 0.5 & 30.4 & 3 & 85 & 2 \\
\hline 350 & Birmingham & $\mathrm{AL}$ & 2.8 & 21 & 3 & 92 & 3 \\
\hline 351 & Birmingham & $\mathrm{AL}$ & 2.8 & 21 & 3 & 92 & 3 \\
\hline 352 & Birmingham & $\mathrm{AL}$ & 2.8 & 21 & 3 & 92 & 3 \\
\hline 353 & ZIP_NOT_ASSIGNED & & 0 & 0 & 0 & 0 & 0 \\
\hline 354 & Tuscaloosa & $\mathrm{AL}$ & 2.8 & 24 & 3 & 83 & 3 \\
\hline 355 & Jasper & AL & 3.2 & 18 & 3 & 75 & 3 \\
\hline 356 & Muscle Shoals & $\mathrm{AL}$ & 3.2 & 20.7 & 3 & 81 & 3 \\
\hline 357 & Huntsville & $\mathrm{AL}$ & 3.3 & 18.6 & 3 & 89 & 3 \\
\hline 358 & Huntsville & $\mathrm{AL}$ & 3.3 & 18.6 & 3 & 89 & 3 \\
\hline 359 & Gadsden & $\mathrm{AL}$ & 3.2 & 17.1 & 3 & 85 & 3 \\
\hline 360 & Montgomery & $\mathrm{AL}$ & 2.2 & 24.6 & 3 & 84 & 3 \\
\hline 361 & Montgomery & $\mathrm{AL}$ & 2.2 & 24.6 & 3 & 84 & 3 \\
\hline 362 & Anniston & $\mathrm{AL}$ & 2.8 & 18.2 & 3 & 73 & 3 \\
\hline 363 & Ozark & $\mathrm{AL}$ & 1.8 & 24.6 & 3 & 76 & 3 \\
\hline 364 & Evergreen & $\mathrm{AL}$ & 2.1 & 22.2 & 3 & 77 & 3 \\
\hline 365 & Mobile & $\mathrm{AL}$ & 1.7 & 28.2 & 3 & 86 & 2 \\
\hline 366 & Mobile & $\mathrm{AL}$ & 1.7 & 28.2 & 3 & 86 & 2 \\
\hline 367 & Selma & $\mathrm{AL}$ & 2.2 & 26.5 & 3 & 78 & 3 \\
\hline 368 & Opelika & $\mathrm{AL}$ & 2.7 & 19.2 & 3 & 85 & 3 \\
\hline 369 & Butler & $\mathrm{AL}$ & 2.7 & 23.8 & 3 & 78 & 3 \\
\hline 370 & Nashville & TN & 3.7 & 18.5 & 3 & 83 & 4 \\
\hline 371 & Nashville & TN & 3.7 & 18.5 & 3 & 83 & 4 \\
\hline 372 & Nashville & $\mathrm{TN}$ & 3.7 & 18.5 & 3 & 83 & 4 \\
\hline 373 & Chattanooga & TN & 3.4 & 17 & 3 & 82 & 4 \\
\hline 374 & Chattanooga & TN & 3.4 & 17 & 3 & 82 & 4 \\
\hline 375 & Memphis & TN & 3 & 24.5 & 3 & 86 & 4 \\
\hline 376 & Kingsport & TN & 4.2 & 8.8 & 3 & 79 & 4 \\
\hline 377 & Knoxville & TN & 3.7 & 15 & 3 & 78 & 4 \\
\hline 378 & Knoxville & $\mathrm{TN}$ & 3.7 & 15 & 3 & 78 & 4 \\
\hline
\end{tabular}




\begin{tabular}{|c|c|c|c|c|c|c|c|}
\hline Zip & Location & State & HDD & CDH & FER & ICF & IECC \\
\hline 379 & Knoxville & $\mathrm{TN}$ & 3.7 & 15 & 3 & 78 & 4 \\
\hline 380 & Dyersburg & $\mathrm{TN}$ & 3.3 & 20.2 & 3 & 86 & 3 \\
\hline 381 & Memphis & $\mathrm{TN}$ & 3 & 24.5 & 3 & 86 & 3 \\
\hline 382 & Union City & $\mathrm{TN}$ & 4.3 & 15 & 3 & 76 & 4 \\
\hline 383 & Jackson & $\mathrm{TN}$ & 3.5 & 18 & 3 & 72 & 4 \\
\hline 384 & Columbia & TN & 4.2 & 16 & 3 & 79 & 4 \\
\hline 385 & Cookeville & TN & 4.2 & 7 & 3 & 77 & 4 \\
\hline 386 & Batesville & MS & 3.3 & 20.6 & 3 & 68 & 3 \\
\hline 387 & Greenville & MS & 2.7 & 25.5 & 3 & 74 & 3 \\
\hline 388 & Tupelo & MS & 3.1 & 23 & 3 & 71 & 3 \\
\hline 389 & Grenada & MS & 3.1 & 26 & 3 & 67 & 3 \\
\hline 390 & Jackson & $\mathrm{MS}$ & 2.4 & 25.2 & 3 & 74 & 3 \\
\hline 391 & Jackson & MS & 2.4 & 25.2 & 3 & 74 & 3 \\
\hline 392 & Jackson & MS & 2.4 & 25.2 & 3 & 74 & 3 \\
\hline 393 & Meridian & MS & 2.4 & 23.8 & 3 & 73 & 3 \\
\hline 394 & Hattiesburg & MS & 2 & 24.3 & 3 & 68 & 2 \\
\hline 395 & Gulfport & MS & 1.5 & 27.5 & 3 & 75 & 2 \\
\hline 396 & McComb & MS & 1.9 & 22.8 & 3 & 74 & 3 \\
\hline 397 & Columbus & MS & 2.7 & 21.8 & 3 & 68 & 3 \\
\hline 398 & Albany & GA & 2.1 & 26.5 & 3 & 83 & 3 \\
\hline 399 & Atlanta & GA & 2.8 & 16.8 & 3 & 92 & 3 \\
\hline 400 & Louisville & $\mathrm{KY}$ & 4.4 & 13.3 & 3 & 84 & 4 \\
\hline 401 & Hardinsburg & KY & 4.4 & 13.8 & 3 & 84 & 4 \\
\hline 402 & Louisville & KY & 4.4 & 13.3 & 3 & 84 & 4 \\
\hline 403 & Mt. Sterling & KY & 4.9 & 10 & 3 & 95 & 4 \\
\hline 404 & Berea & KY & 4.2 & 11.8 & 3 & 95 & 4 \\
\hline 405 & Lexington & KY & 4.7 & 11.2 & 3 & 95 & 4 \\
\hline 406 & Frankfort & $\mathrm{KY}$ & 5.1 & 9.7 & 3 & 90 & 4 \\
\hline 407 & Williamsburg & $\mathrm{KY}$ & 4.6 & 11.4 & 3 & 80 & 4 \\
\hline 408 & Baxter & KY & 4.6 & 7.8 & 3 & 80 & 4 \\
\hline 409 & Middlesboro & $\mathrm{KY}$ & 4.6 & 9.5 & 3 & 80 & 4 \\
\hline 410 & Covington & $\mathrm{KY}$ & 5.1 & 9.3 & 3 & 98 & 4 \\
\hline 411 & Ashland & KY & 5.2 & 11.4 & 3 & 93 & 4 \\
\hline 412 & Paintsville & $\mathrm{KY}$ & 4.4 & 8.1 & 3 & 93 & 4 \\
\hline 413 & Jackson & $\mathrm{KY}$ & 4.4 & 6.3 & 3 & 80 & 4 \\
\hline 414 & West Liberty & $\mathrm{KY}$ & 5.2 & 8.1 & 3 & 80 & 4 \\
\hline 415 & Pikeville & KY & 4.5 & 9.5 & 3 & 79 & 4 \\
\hline 416 & Eastern & KY & 4.8 & 8.1 & 3 & 79 & 4 \\
\hline 417 & Hyden & KY & 4.7 & 9.5 & 3 & 79 & 4 \\
\hline 418 & Hyden & KY & 4.7 & 9.5 & 3 & 79 & 4 \\
\hline 419 & ZIP_NOT_ASSIGNED & & 0 & 0 & 0 & 0 & 0 \\
\hline 420 & Paducah & KY & 4.3 & 16.7 & 3 & 92 & 4 \\
\hline 421 & Bowling Green & KY & 4.2 & 14.7 & 3 & 87 & 4 \\
\hline 422 & Russellville & KY & 4.4 & 14.4 & 3 & 87 & 4 \\
\hline 423 & Owensboro & KY & 4.2 & 14.5 & 3 & 90 & 4 \\
\hline 424 & Henderson & KY & 4.4 & 14.2 & 3 & 95 & 4 \\
\hline 425 & Somerset & KY & 4.4 & 9.2 & 3 & 82 & 4 \\
\hline 426 & Monticello & KY & 4.6 & 9.2 & 3 & 82 & 4 \\
\hline
\end{tabular}




\begin{tabular}{|c|c|c|c|c|c|c|c|}
\hline Zip & Location & State & HDD & $\mathrm{CDH}$ & FER & ICF & IECC \\
\hline 427 & Elizabethtown & KY & 4.9 & 11.8 & 3 & 84 & 4 \\
\hline 428 & ZIP_NOT_ASSIGNED & & 0 & 0 & 0 & 0 & 0 \\
\hline 429 & ZIP NOT ASSIGNED & & 0 & 0 & 0 & 0 & 0 \\
\hline 430 & Columbus & $\mathrm{OH}$ & 5.5 & 7.5 & 2 & 97 & 5 \\
\hline 431 & Lancaster & $\mathrm{OH}$ & 5.9 & 8.2 & 2 & 97 & 5 \\
\hline 432 & Columbus & $\mathrm{OH}$ & 5.5 & 7.5 & 2 & 97 & 5 \\
\hline 433 & Marion & $\mathrm{OH}$ & 6.3 & 6.5 & 2 & 94 & 5 \\
\hline 434 & Bowling Green & $\mathrm{OH}$ & 6.5 & 6.6 & 2 & 103 & 5 \\
\hline 435 & Napoleon & $\mathrm{OH}$ & 6.6 & 7.1 & 2 & 103 & 5 \\
\hline 436 & Toledo & $\mathrm{OH}$ & 6.5 & 5.1 & 2 & 103 & 5 \\
\hline 437 & Cambridge & $\mathrm{OH}$ & 5.3 & 5.5 & 2 & 95 & 5 \\
\hline 438 & Coshocton & $\mathrm{OH}$ & 5.7 & 6.6 & 2 & 95 & 5 \\
\hline 439 & Stuebenville & $\mathrm{OH}$ & 5.6 & 5.7 & 2 & 103 & 5 \\
\hline 440 & Cleveland & $\mathrm{OH}$ & 6.1 & 4.8 & 2 & 103 & 5 \\
\hline 441 & Cleveland & $\mathrm{OH}$ & 6.1 & 4.8 & 2 & 105 & 5 \\
\hline 442 & Akron & $\mathrm{OH}$ & 5.8 & 4.8 & 2 & 100 & 5 \\
\hline 443 & Akron & $\mathrm{OH}$ & 5.8 & 4.8 & 2 & 100 & 5 \\
\hline 444 & Youngstown & $\mathrm{OH}$ & 6.5 & 3 & 2 & 100 & 5 \\
\hline 445 & Youngstown & $\mathrm{OH}$ & 6.5 & 3 & 2 & 100 & 5 \\
\hline 446 & Canton & $\mathrm{OH}$ & 6.2 & 4.8 & 2 & 98 & 5 \\
\hline 447 & Canton & $\mathrm{OH}$ & 6.2 & 4.8 & 2 & 98 & 5 \\
\hline 448 & Mansfield & $\mathrm{OH}$ & 6.4 & 4.9 & 2 & 99 & 5 \\
\hline 449 & Mansfield & $\mathrm{OH}$ & 6.4 & 4.9 & 2 & 99 & 5 \\
\hline 450 & Fairfield & $\mathrm{OH}$ & 5.3 & 9.5 & 2 & 96 & 4 \\
\hline 451 & Hillsboro & $\mathrm{OH}$ & 5.6 & 7.1 & 2 & 95 & 4 \\
\hline 452 & Cincinnati & $\mathrm{OH}$ & 4.9 & 10.7 & 2 & 95 & 4 \\
\hline 453 & Dayton & $\mathrm{OH}$ & 5.7 & 8.3 & 2 & 96 & 5 \\
\hline 454 & Dayton & $\mathrm{OH}$ & 5.7 & 8.3 & 2 & 96 & 5 \\
\hline 455 & Springfield & $\mathrm{OH}$ & 5.9 & 8.3 & 2 & 96 & 5 \\
\hline 456 & Chillicothe & $\mathrm{OH}$ & 5.6 & 8 & 2 & 96 & 4 \\
\hline 457 & Athens & $\mathrm{OH}$ & 5.7 & 5.6 & 2 & 94 & 5 \\
\hline 458 & Lima & $\mathrm{OH}$ & 5.9 & 7.5 & 2 & 98 & 5 \\
\hline 459 & Cincinnati & $\mathrm{OH}$ & 4.9 & 10.7 & 2 & 95 & 4 \\
\hline 460 & Anderson & IN & 5.8 & 9.3 & 2 & 92 & 5 \\
\hline 461 & Greencastle & IN & 5.6 & 10.2 & 2 & 93 & 5 \\
\hline 462 & Indianapolis & IN & 5.5 & 9.1 & 2 & 93 & 5 \\
\hline 463 & Valparaiso & IN & 6.3 & 5.6 & 2 & 100 & 5 \\
\hline 464 & Gary & IN & 6.5 & 9.1 & 2 & 100 & 5 \\
\hline 465 & Plymouth & IN & 6.5 & 9.6 & 2 & 92 & 5 \\
\hline 466 & South Bend & IN & 6.3 & 6.6 & 2 & 92 & 5 \\
\hline 467 & Fort Wayne & IN & 6.2 & 6.8 & 2 & 93 & 5 \\
\hline 468 & Fort Wayne & IN & 6.2 & 6.8 & 2 & 93 & 5 \\
\hline 469 & Kokomo & IN & 6.4 & 11.1 & 2 & 91 & 5 \\
\hline 470 & Brookville & IN & 5.7 & 7 & 2 & 86 & 5 \\
\hline 471 & Scottsburg & IN & 5.1 & 10.2 & 2 & 78 & 4 \\
\hline 472 & Columbus & IN & 5.4 & 8.8 & 2 & 87 & 4 \\
\hline 473 & Muncie & IN & 6 & 7.1 & 2 & 88 & 5 \\
\hline 474 & Bloomington & IN & 5.3 & 10.7 & 2 & 87 & 4 \\
\hline
\end{tabular}




\begin{tabular}{|c|c|c|c|c|c|c|c|}
\hline Zip & Location & State & HDD & CDH & FER & ICF & IECC \\
\hline 475 & Washington & IN & 4.6 & 13.3 & 2 & 90 & 4 \\
\hline 476 & Evansville & IN & 4.6 & 15 & 2 & 91 & 4 \\
\hline 477 & Evansville & IN & 4.6 & 15 & 2 & 91 & 4 \\
\hline 478 & Terre Haute & IN & 5.4 & 9.5 & 2 & 90 & 4 \\
\hline 479 & Lafayette & IN & 6.2 & 7.7 & 2 & 86 & 5 \\
\hline 480 & Port Huron & MI & 6.8 & 5.3 & 2 & 104 & 5 \\
\hline 481 & Ann Arbor & MI & 6.5 & 6.1 & 2 & 107 & 5 \\
\hline 482 & Detroit & MI & 6.4 & 4.9 & 2 & 107 & 5 \\
\hline 483 & Milford & MI & 7 & 3.6 & 2 & 104 & 5 \\
\hline 484 & Flint & MI & 7 & 2.9 & 2 & 96 & 5 \\
\hline 485 & Flint & MI & 7 & 2.9 & 2 & 96 & 5 \\
\hline 486 & Saginaw & MI & 7.1 & 3.3 & 2 & 96 & 5 \\
\hline 487 & Caro & MI & 7 & 3 & 2 & 96 & 5 \\
\hline 488 & Lansing & MI & 7.1 & 4.1 & 2 & 97 & 5 \\
\hline 489 & Lansing & MI & 7.1 & 4.1 & 2 & 97 & 5 \\
\hline 490 & Kalamazoo & MI & 6.2 & 6.3 & 2 & 91 & 5 \\
\hline 491 & Eau Claire & MI & 6.2 & 5.8 & 2 & 91 & 5 \\
\hline 492 & Jackson & MI & 6.9 & 4.8 & 2 & 94 & 5 \\
\hline 493 & Grand Rapids & MI & 6.9 & 4.6 & 2 & 78 & 5 \\
\hline 494 & Muskegon & MI & 6.9 & 2.9 & 2 & 85 & 5 \\
\hline 495 & Grand Rapids & $\mathrm{MI}$ & 6.9 & 4.6 & 2 & 78 & 5 \\
\hline 496 & Traverse City & MI & 7.6 & 3 & 2 & 81 & 6 \\
\hline 497 & Cheboygan & MI & 8.4 & 2 & 2 & 80 & 7 \\
\hline 498 & Iron Mountain & MI & 8.8 & 1.4 & 2 & 89 & 6 \\
\hline 499 & Houghton & MI & 9.1 & 1 & 2 & 89 & 7 \\
\hline 500 & Ames & IA & 6.8 & 7.5 & 2 & 92 & 5 \\
\hline 501 & Ames & IA & 6.8 & 7.5 & 2 & 92 & 5 \\
\hline 502 & Ames & IA & 6.8 & 7.5 & 2 & 92 & 5 \\
\hline 503 & Des Moines & IA & 6.4 & 10.5 & 2 & 92 & 5 \\
\hline 504 & Mason City & IA & 8 & 6 & 2 & 78 & 6 \\
\hline 505 & Fort Dodge & IA & 7.5 & 8 & 2 & 74 & 6 \\
\hline 506 & Waterloo & IA & 7.3 & 6.6 & 2 & 79 & 6 \\
\hline $\mathbf{5 0 7}$ & Waterloo & IA & 7.3 & 6.6 & 2 & 79 & 6 \\
\hline 508 & Creston & IA & 6.8 & 9.5 & 2 & 82 & 5 \\
\hline 509 & Des Moines & IA & 6.4 & 10.5 & 2 & 92 & 5 \\
\hline 510 & Hawarden & IA & 7.6 & 9.2 & 2 & 81 & 6 \\
\hline 511 & Sioux City & IA & 6.9 & 10.1 & 2 & 81 & 5 \\
\hline 512 & Sheldon & IA & 8.1 & 6.6 & 2 & 79 & 6 \\
\hline 513 & Spencer & IA & 7.7 & 6.1 & 2 & 77 & 6 \\
\hline 514 & Carroll & IA & 7.1 & 8.2 & 2 & 80 & 5 \\
\hline 515 & Red Oak & IA & 6.5 & 11.5 & 2 & 85 & 5 \\
\hline 516 & Shenandoah & IA & 6.2 & 12.7 & 2 & 76 & 5 \\
\hline 517 & ZIP NOT ASSIGNED & & 0 & 0 & 0 & 0 & 0 \\
\hline 518 & ZIP_NOT_ASSIGNED & & 0 & 0 & 0 & 0 & 0 \\
\hline 519 & ZIP NOT ASSIGNED & & 0 & 0 & 0 & 0 & 0 \\
\hline 520 & Dubuque & IA & 7.3 & 4.7 & 2 & 88 & 6 \\
\hline 521 & Decorah & IA & 7.4 & 5.3 & 2 & 78 & 6 \\
\hline 522 & Cedar Rapids & IA & 6.8 & 7.9 & 2 & 92 & 5 \\
\hline
\end{tabular}




\begin{tabular}{|c|c|c|c|c|c|c|c|}
\hline Zip & Location & State & HDD & CDH & FER & ICF & IECC \\
\hline 523 & Cedar Rapids & IA & 6.8 & 7.9 & 2 & 92 & 5 \\
\hline 524 & Cedar Rapids & IA & 6.8 & 7.9 & 2 & 92 & 5 \\
\hline 525 & Ottumwa & IA & 6.1 & 10 & 2 & 82 & 5 \\
\hline 526 & Burlington & IA & 5.9 & 10 & 2 & 84 & 5 \\
\hline 527 & Tipton & IA & 7.1 & 8.8 & 2 & 96 & 5 \\
\hline 528 & Davenport & IA & 6.2 & 10 & 2 & 96 & 5 \\
\hline 529 & ZIP NOT ASSIGNED & & 0 & 0 & 0 & 0 & 0 \\
\hline 530 & Sheboygan & WI & 7.1 & 2.7 & 2 & 104 & 6 \\
\hline 531 & Burlington & WI & 7.5 & 3.7 & 2 & 100 & 6 \\
\hline 532 & Milwaukee & WI & 7.1 & 3.3 & 2 & 104 & 6 \\
\hline 533 & Not used & $\mathrm{NU}$ & 0 & 0 & 0 & 0 & 0 \\
\hline 534 & Racine & WI & 7 & 5.2 & 2 & 100 & 6 \\
\hline 535 & Madison & WI & 7.5 & 3.3 & 2 & 99 & 6 \\
\hline 536 & Not used & $\mathrm{NU}$ & 0 & 0 & 0 & 0 & 0 \\
\hline 537 & Madison & WI & 7.5 & 3.3 & 2 & 95 & 6 \\
\hline 538 & Platteville & WI & 7.5 & 5.6 & 2 & 92 & 6 \\
\hline 539 & Portage & WI & 7.8 & 5.5 & 2 & 97 & 6 \\
\hline 540 & River Falls & WI & 8.4 & 4.6 & 2 & 99 & 6 \\
\hline 541 & Marinette & WI & 7.9 & 3.7 & 2 & 95 & 6 \\
\hline 542 & Manitowac & WI & 7.6 & 2.2 & 2 & 95 & 6 \\
\hline 543 & Green Bay & WI & 8 & 2.5 & 2 & 95 & 6 \\
\hline 544 & Wausau & WI & 8.2 & 2.5 & 2 & 92 & 6 \\
\hline 545 & Rhinelander & WI & 9.1 & 2.3 & 2 & 93 & 7 \\
\hline 546 & La Crosse & WI & 7.3 & 6.8 & 2 & 94 & 6 \\
\hline 547 & Eau Claire & WI & 8.2 & 3.9 & 2 & 94 & 6 \\
\hline 548 & Spooner & WI & 8.5 & 2.5 & 2 & 99 & 7 \\
\hline 549 & Oshkosh & WI & 7.6 & 3.7 & 2 & 95 & 6 \\
\hline 550 & Forest Lake & $\mathrm{MN}$ & 7.9 & 6.8 & 2 & 114 & 6 \\
\hline 551 & Saint Paul & $\mathrm{MN}$ & 7.6 & 6.8 & 2 & 114 & 6 \\
\hline 552 & ZIP_NOT_ASSIGNED & & 0 & 0 & 0 & 0 & 0 \\
\hline 553 & Hutchinson & $\mathrm{MN}$ & 8.3 & 6.8 & 2 & 115 & 6 \\
\hline 554 & Minneapolis & $\mathrm{MN}$ & 7.9 & 6.8 & 2 & 115 & 6 \\
\hline 555 & Maple Plain & $\mathrm{MN}$ & 8 & 5.5 & 2 & 115 & 6 \\
\hline 556 & Two Harbors & $\mathrm{MN}$ & 9.1 & 1 & 2 & 108 & 7 \\
\hline 557 & Grand Rapids & $\mathrm{MN}$ & 9.5 & 1.7 & 2 & 108 & 7 \\
\hline 558 & Duluth & $\mathrm{MN}$ & 9.7 & 0.8 & 2 & 108 & 7 \\
\hline 559 & Rochester & $\mathrm{MN}$ & 8.3 & 3.9 & 2 & 102 & 7 \\
\hline 560 & Mankato & $\mathrm{MN}$ & 8 & 5 & 2 & 100 & 6 \\
\hline 561 & Windom & $\mathrm{MN}$ & 8.1 & 7.2 & 2 & 79 & 6 \\
\hline 562 & Willmar & $\mathrm{MN}$ & 8.6 & 4.7 & 2 & 83 & 6 \\
\hline 563 & Saint Cloud & $\mathrm{MN}$ & 8.8 & 3 & 2 & 107 & 6 \\
\hline 564 & Brainerd & $\mathrm{MN}$ & 9.5 & 3.5 & 2 & 103 & 7 \\
\hline 565 & Detroit Lakes & $\mathrm{MN}$ & 9.2 & 2.3 & 2 & 94 & 7 \\
\hline 566 & International Falls & $\mathrm{MN}$ & 10.2 & 1.6 & 2 & 98 & 7 \\
\hline 567 & Thief River Falls & $\mathrm{MN}$ & 9.8 & 3 & 2 & 94 & 7 \\
\hline 568 & ZIP_NOT_ASSIGNED & & 0 & 0 & 0 & 0 & 0 \\
\hline 569 & ZIP_NOT_ASSIGNED & & 0 & 0 & 0 & 0 & 0 \\
\hline 570 & Madison & SD & 8.4 & 8.6 & 2 & 81 & 6 \\
\hline
\end{tabular}




\begin{tabular}{|c|c|c|c|c|c|c|c|}
\hline Zip & Location & State & HDD & CDH & FER & ICF & IECC \\
\hline 571 & Sioux Falls & SD & 7.8 & 8.6 & 2 & 81 & 6 \\
\hline 572 & Watertown & SD & 8.8 & 4.9 & 2 & 79 & 6 \\
\hline 573 & Mitchell & SD & 7.6 & 10.3 & 2 & 78 & 6 \\
\hline 574 & Aberdeen & SD & 8.3 & 6.5 & 2 & 79 & 6 \\
\hline 575 & Pierre & SD & 7.3 & 10.4 & 2 & 78 & 6 \\
\hline 576 & Mobridge & SD & 8 & 7.8 & 2 & 79 & 6 \\
\hline 577 & Rapid City & SD & 7.2 & 8.2 & 2 & 79 & 6 \\
\hline 578 & ZIP_NOT_ASSIGNED & & 0 & 0 & 0 & 0 & 0 \\
\hline 579 & ZIP_NOT_ASSIGNED & & 0 & 0 & 0 & 0 & 0 \\
\hline 580 & Valley City & ND & 9.6 & 3.8 & 2 & 81 & 7 \\
\hline 581 & Fargo & ND & 9.1 & 4.3 & 2 & 81 & 7 \\
\hline 582 & Grand Forks & ND & 9.5 & 4.1 & 2 & 82 & 7 \\
\hline 583 & Devils Lake & ND & 9.4 & 3.1 & 2 & 82 & 7 \\
\hline 584 & Jamestown & ND & 9 & 4 & 2 & 79 & 6 \\
\hline 585 & Bismarck & ND & 8.8 & 4.6 & 2 & 81 & 6 \\
\hline 586 & Dickinson & ND & 8.6 & 4 & 2 & 81 & 6 \\
\hline 587 & Minot & ND & 9 & 4 & 2 & 82 & 7 \\
\hline 588 & Williston & ND & 9 & 4 & 2 & 81 & 7 \\
\hline 589 & ZIP_NOT_ASSIGNED & & 0 & 0 & 0 & 0 & 0 \\
\hline 590 & Roundup & MT & 7 & 5.5 & 4 & 86 & 6 \\
\hline 591 & Billings & MT & 7 & 6 & 4 & 86 & 6 \\
\hline 592 & Wolf Point & MT & 8.4 & 4.8 & 4 & 85 & 6 \\
\hline 593 & Miles City & MT & 7.6 & 10 & 4 & 86 & 6 \\
\hline 594 & Great Falls & MT & 7.8 & 3.6 & 4 & 86 & 6 \\
\hline 595 & Havre & MT & 8.3 & 4 & 4 & 84 & 6 \\
\hline 596 & Helena & MT & 8 & 2.5 & 4 & 86 & 6 \\
\hline 597 & Butte & MT & 9.4 & 0.9 & 4 & 86 & 6 \\
\hline 598 & Missoula & MT & 7.6 & 1.1 & 4 & 86 & 6 \\
\hline 599 & Kalispell & MT & 8.2 & 1.7 & 4 & 84 & 6 \\
\hline 600 & Antioch & IL & 6.9 & 5.6 & 2 & 110 & 5 \\
\hline 601 & DeKalb & IL & 7 & 7 & 2 & 110 & 5 \\
\hline 602 & Evanston & IL & 6.5 & 6.6 & 2 & 110 & 5 \\
\hline 603 & Oak Park & IL & 6.5 & 6.6 & 2 & 110 & 5 \\
\hline 604 & Joliet & IL & 6.5 & 6.4 & 2 & 109 & 5 \\
\hline 605 & Aurora & IL & 6.9 & 6.4 & 2 & 110 & 5 \\
\hline 606 & Chicago & IL & 6.5 & 9.7 & 2 & 114 & 5 \\
\hline 607 & Chicago & IL & 6.5 & 9.7 & 2 & 114 & 5 \\
\hline 608 & Chicago & IL & 6.5 & 9.7 & 2 & 114 & 5 \\
\hline 609 & Kankakee & IL & 6.4 & 8.8 & 2 & 108 & 5 \\
\hline 610 & Freeport & IL & 7.3 & 6.5 & 2 & 105 & 5 \\
\hline 611 & Rockford & IL & 6.9 & 6.5 & 2 & 105 & 5 \\
\hline 612 & Rock Island & IL & 6.2 & 10 & 2 & 96 & 5 \\
\hline 613 & Ottawa & $\mathrm{IL}$ & 6.3 & 10 & 2 & 97 & 5 \\
\hline 614 & Galesburg & $\mathrm{IL}$ & 6.3 & 8.9 & 2 & 98 & 5 \\
\hline 615 & Peoria & IL & 6.1 & 9.5 & 2 & 98 & 5 \\
\hline 616 & Peoria & IL & 6.1 & 9.5 & 2 & 98 & 5 \\
\hline 617 & Normal & $\mathrm{IL}$ & 6.2 & 9.5 & 2 & 99 & 5 \\
\hline 618 & Champaign/Urbana & IL & 5.9 & 9.9 & 2 & 99 & 5 \\
\hline
\end{tabular}




\begin{tabular}{|c|c|c|c|c|c|c|c|}
\hline Zip & Location & State & HDD & $\mathrm{CDH}$ & FER & ICF & IECC \\
\hline 619 & Paris & IL & 5.7 & 11 & 2 & 99 & 5 \\
\hline 620 & Jerseyville & IL & 5.4 & 12.8 & 2 & 97 & 4 \\
\hline 621 & ZIP NOT ASSIGNED & & 0 & 0 & 0 & 0 & 0 \\
\hline 622 & Waterloo & IL & 4.9 & 13.5 & 2 & 97 & 4 \\
\hline 623 & Quincy & IL & 5.7 & 12.2 & 2 & 96 & 5 \\
\hline 624 & Effingham & IL & 5.5 & 13.2 & 2 & 94 & 5 \\
\hline 625 & Decatur & IL & 5.5 & 12.1 & 2 & 97 & 5 \\
\hline 626 & Jacksonville & IL & 5.9 & 12.3 & 2 & 98 & 5 \\
\hline 627 & Springfield & IL & 5.6 & 12.4 & 2 & 98 & 5 \\
\hline 628 & Mount Vernon & IL & 5.2 & 13.5 & 2 & 96 & 4 \\
\hline 629 & Carbondale & IL & 4.9 & 14.1 & 2 & 91 & 4 \\
\hline 630 & Union & $\mathrm{MO}$ & 4.6 & 13.9 & 2 & 101 & 4 \\
\hline 631 & Saint Louis & $\mathrm{MO}$ & 4.8 & 17.8 & 2 & 101 & 4 \\
\hline 632 & ZIP NOT ASSIGNED & & 0 & 0 & 0 & 0 & 0 \\
\hline 633 & Saint Charles & $\mathrm{MO}$ & 5.2 & 17.1 & 2 & 97 & 4 \\
\hline 634 & Hannibal & MO & 5.7 & 11.8 & 2 & 92 & 5 \\
\hline 635 & Kirksville & MO & 6.1 & 9.9 & 2 & 92 & 5 \\
\hline 636 & Farmington & MO & 5.1 & 12.2 & 2 & 95 & 4 \\
\hline 637 & Cape Girardeau & MO & 4.3 & 16.8 & 2 & 89 & 4 \\
\hline 638 & Sikeston & MO & 4.3 & 16.9 & 2 & 88 & 4 \\
\hline 639 & Poplar Bluff & $\mathrm{MO}$ & 4.2 & 17.2 & 2 & 90 & 4 \\
\hline 640 & Independence & $\mathrm{MO}$ & 5.4 & 17.5 & 2 & 100 & 4 \\
\hline 641 & Kansas City & $\mathrm{MO}$ & 5.2 & 17.5 & 2 & 100 & 4 \\
\hline 642 & ZIP NOT ASSIGNED & & 0 & 0 & 0 & 0 & 0 \\
\hline 643 & ZIP NOT ASSIGNED & & 0 & 0 & 0 & 0 & 0 \\
\hline 644 & Maryville & MO & 6.2 & 11.6 & 2 & 94 & 5 \\
\hline 645 & Saint Joseph & MO & 5.3 & 16.1 & 2 & 94 & 5 \\
\hline 646 & Chillicothe & MO & 5.8 & 14.4 & 2 & 89 & 5 \\
\hline 647 & Nevada & $\mathrm{MO}$ & 4.8 & 20.3 & 2 & 97 & 4 \\
\hline 648 & Joplin & $\mathrm{MO}$ & 4.3 & 20.8 & 2 & 84 & 4 \\
\hline 649 & Kansas City & $\mathrm{MO}$ & 5.2 & 17.5 & 2 & 100 & 4 \\
\hline 650 & Jefferson City & MO & 5.2 & 15 & 2 & 87 & 4 \\
\hline 651 & Jefferson City & MO & 5.2 & 15 & 2 & 87 & 4 \\
\hline 652 & Columbia & MO & 5.2 & 14.5 & 2 & 88 & 4 \\
\hline 653 & Sedalia & MO & 5.3 & 17.2 & 2 & 88 & 4 \\
\hline 654 & Rolla & $\mathrm{MO}$ & 4.9 & 12.8 & 2 & 79 & 4 \\
\hline 655 & Lebanon & MO & 4.6 & 15.8 & 2 & 79 & 4 \\
\hline 656 & Springfield & $\mathrm{MO}$ & 4.6 & 16.3 & 2 & 88 & 4 \\
\hline 657 & Springfield & $\mathrm{MO}$ & 4.6 & 16.3 & 2 & 88 & 4 \\
\hline 658 & Springfield & $\mathrm{MO}$ & 4.6 & 16.3 & 2 & 88 & 4 \\
\hline 659 & ZIP NOT ASSIGNED & & 0 & 0 & 0 & 0 & 0 \\
\hline 660 & Lawrence & $\mathrm{KS}$ & 4.7 & 18.7 & 2 & 98 & 4 \\
\hline 661 & Kansas City & $\mathrm{KS}$ & 5.2 & 17.5 & 2 & 98 & 4 \\
\hline 662 & Shawnee & $\mathrm{KS}$ & 5.2 & 17.5 & 2 & 98 & 4 \\
\hline 663 & ZIP NOT ASSIGNED & & 0 & 0 & 0 & 0 & 0 \\
\hline 664 & Topeka & $\mathrm{KS}$ & 5.2 & 16.6 & 2 & 86 & 4 \\
\hline 665 & Manhattan & $\mathrm{KS}$ & 5.1 & 18.8 & 2 & 86 & 4 \\
\hline 666 & Topeka & KS & 5.2 & 16.6 & 2 & 86 & 4 \\
\hline
\end{tabular}




\begin{tabular}{|c|c|c|c|c|c|c|c|}
\hline Zip & Location & State & HDD & CDH & FER & ICF & IECC \\
\hline 667 & Fort Scott & KS & 4.7 & 24.1 & 2 & 85 & 4 \\
\hline 668 & Emporia & KS & 5.2 & 17.4 & 2 & 73 & 4 \\
\hline 669 & Concordia & $\mathrm{KS}$ & 5.5 & 16.7 & 2 & 81 & 5 \\
\hline 670 & Wichita & $\mathrm{KS}$ & 4.8 & 21.2 & 2 & 83 & 4 \\
\hline 671 & Wichita & KS & 4.8 & 21.2 & 2 & 83 & 4 \\
\hline 672 & Wichita & $\mathrm{KS}$ & 4.8 & 21.2 & 2 & 83 & 4 \\
\hline 673 & Independence & $\mathrm{KS}$ & 4.5 & 20.3 & 2 & 83 & 4 \\
\hline 674 & Salina & $\mathrm{KS}$ & 5 & 19.8 & 2 & 80 & 4 \\
\hline 675 & Hutchinson & $\mathrm{KS}$ & 5.1 & 21.9 & 2 & 76 & 4 \\
\hline 676 & Hays & KS & 5.5 & 16.3 & 2 & 82 & 5 \\
\hline 677 & Colby & $\mathrm{KS}$ & 6.3 & 11.9 & 2 & 81 & 5 \\
\hline 678 & Dodge City & $\mathrm{KS}$ & 5 & 18.5 & 2 & 82 & 4 \\
\hline 679 & Liberal & $\mathrm{KS}$ & 4.8 & 18.5 & 2 & 74 & 4 \\
\hline 680 & Omaha & $\mathrm{NE}$ & 6.3 & 12 & 2 & 83 & 5 \\
\hline 681 & Omaha & $\mathrm{NE}$ & 6.3 & 12 & 2 & 83 & 5 \\
\hline 682 & ZIP_NOT_ASSIGNED & & 0 & 0 & 0 & 0 & 0 \\
\hline 683 & Lincoln & $\mathrm{NE}$ & 6.2 & 13.6 & 2 & 81 & 5 \\
\hline 684 & Lincoln & $\mathrm{NE}$ & 6.2 & 13.6 & 2 & 81 & 5 \\
\hline 685 & Lincoln & $\mathrm{NE}$ & 6.2 & 13.6 & 2 & 81 & 5 \\
\hline 686 & Columbus & $\mathrm{NE}$ & 6.4 & 12.7 & 2 & 70 & 5 \\
\hline 687 & Norfolk & $\mathrm{NE}$ & 6.8 & 10.6 & 2 & 78 & 5 \\
\hline 688 & Grand Island & $\mathrm{NE}$ & 6.4 & 12 & 2 & 80 & 5 \\
\hline 689 & Hastings & $\mathrm{NE}$ & 6.2 & 12.6 & 2 & 75 & 5 \\
\hline 690 & McCook & $\mathrm{NE}$ & 6 & 13.6 & 2 & 71 & 5 \\
\hline 691 & North Platte & $\mathrm{NE}$ & 6.8 & 8.5 & 2 & 75 & 5 \\
\hline 692 & Valentine & $\mathrm{NE}$ & 6.8 & 8.2 & 2 & 69 & 5 \\
\hline 693 & Alliance & $\mathrm{NE}$ & 6.8 & 6.4 & 2 & 75 & 5 \\
\hline 694 & ZIP NOT ASSIGNED & & 0 & 0 & 0 & 0 & 0 \\
\hline 695 & ZIP_NOT_ASSIGNED & & 0 & 0 & 0 & 0 & 0 \\
\hline 696 & ZIP_NOT_ASSIGNED & & 0 & 0 & 0 & 0 & 0 \\
\hline 697 & ZIP_NOT_ASSIGNED & & 0 & 0 & 0 & 0 & 0 \\
\hline 698 & ZIP_NOT_ASSIGNED & & 0 & 0 & 0 & 0 & 0 \\
\hline 699 & ZIP NOT ASSIGNED & & 0 & 0 & 0 & 0 & 0 \\
\hline 700 & New Orleans & LA & 1.4 & 28.6 & 3 & 86 & 2 \\
\hline 701 & New Orleans & LA & 1.4 & 28.6 & 3 & 86 & 2 \\
\hline 702 & ZIP_NOT_ASSIGNED & & 0 & 0 & 0 & 0 & 0 \\
\hline 703 & Thibodaux & LA & 1.4 & 27.9 & 3 & 85 & 2 \\
\hline 704 & Hammond & LA & 1.8 & 24.7 & 3 & 85 & 2 \\
\hline 705 & Lafayette & LA & 1.5 & 28.5 & 3 & 83 & 2 \\
\hline 706 & Lake Charles & LA & 1.5 & 28.6 & 3 & 83 & 2 \\
\hline 707 & Baton Rouge & LA & 1.7 & 26.9 & 3 & 81 & 2 \\
\hline 708 & Baton Rouge & LA & 1.7 & 26.9 & 3 & 81 & 2 \\
\hline 709 & ZIP_NOT_ASSIGNED & & 0 & 0 & 0 & 0 & 0 \\
\hline 710 & Shreveport & LA & 2.3 & 28.3 & 3 & 81 & 3 \\
\hline 711 & Shreveport & LA & 2.3 & 28.3 & 3 & 81 & 3 \\
\hline 712 & Monroe & LA & 2.2 & 26.6 & 3 & 82 & 3 \\
\hline 713 & Alexandria & LA & 1.9 & 27.3 & 3 & 81 & 2 \\
\hline 714 & Natchitoches & LA & 2.2 & 28.9 & 3 & 81 & 3 \\
\hline
\end{tabular}




\begin{tabular}{|c|c|c|c|c|c|c|c|}
\hline Zip & Location & State & HDD & CDH & FER & ICF & IECC \\
\hline 715 & ZIP NOT ASSIGNED & & 0 & 0 & 0 & 0 & 0 \\
\hline 716 & Pine Bluff & AR & 2.9 & 26.7 & 3 & 83 & 3 \\
\hline 717 & Camden & AR & 2.9 & 23.7 & 3 & 74 & 3 \\
\hline 718 & Hope & AR & 3.1 & 22.5 & 3 & 79 & 3 \\
\hline 719 & Hot Springs Nat Pk & AR & 3.1 & 26.6 & 3 & 76 & 3 \\
\hline 720 & Little Rock & AR & 3.1 & 23.8 & 3 & 83 & 3 \\
\hline 721 & Little Rock & $\mathrm{AR}$ & 3.1 & 23.8 & 3 & 83 & 3 \\
\hline 722 & Little Rock & $\mathrm{AR}$ & 3.1 & 23.8 & 3 & 83 & 3 \\
\hline 723 & West Memphis & $\mathrm{AR}$ & 3.4 & 24.5 & 3 & 86 & 3 \\
\hline 724 & Jonesboro & AR & 3.7 & 23.2 & 3 & 85 & 3 \\
\hline 725 & Batesville & AR & 4 & 19 & 3 & 80 & 4 \\
\hline 726 & Harrison & $\mathrm{AR}$ & 4.1 & 18.5 & 3 & 80 & 4 \\
\hline 727 & Fayetteville & AR & 4.2 & 16 & 3 & 81 & 4 \\
\hline 728 & Clarksville & $\mathrm{AR}$ & 3.8 & 22.7 & 3 & 80 & 3 \\
\hline 729 & Fort Smith & $\mathrm{AR}$ & 3.4 & 23.5 & 3 & 82 & 3 \\
\hline 730 & Oklahoma City & $\mathrm{OK}$ & 3.7 & 23 & 3 & 84 & 3 \\
\hline 731 & Oklahoma City & $\mathrm{OK}$ & 3.7 & 23 & 3 & 84 & 3 \\
\hline 732 & ZIP_NOT_ASSIGNED & & 0 & 0 & 0 & 0 & 0 \\
\hline 733 & Austin & TX & 1.5 & 35.2 & 3 & 77 & 3 \\
\hline 734 & Ardmore & OK & 3 & 31.7 & 3 & 84 & 3 \\
\hline 735 & Lawton & $\mathrm{OK}$ & 3.3 & 27.1 & 3 & 85 & 3 \\
\hline 736 & Clinton & $\mathrm{OK}$ & 4 & 26.4 & 3 & 84 & 3 \\
\hline 737 & Enid & $\mathrm{OK}$ & 4.3 & 26.1 & 3 & 84 & 3 \\
\hline 738 & Woodward & $\mathrm{OK}$ & 4.7 & 23.2 & 3 & 84 & 3 \\
\hline 739 & Guymon & $\mathrm{OK}$ & 4.6 & 17.5 & 3 & 72 & 4 \\
\hline 740 & Bartlesville & $\mathrm{OK}$ & 3.7 & 22.7 & 3 & 83 & 3 \\
\hline 741 & Tulsa & $\mathrm{OK}$ & 3.6 & 26.5 & 3 & 83 & 3 \\
\hline 742 & ZIP_NOT_ASSIGNED & & 0 & 0 & 0 & 0 & 0 \\
\hline 743 & Vinita & $\mathrm{OK}$ & 3.9 & 23.2 & 3 & 85 & 3 \\
\hline 744 & Muskogee & $\mathrm{OK}$ & 3.7 & 25.7 & 3 & 78 & 3 \\
\hline 745 & McAlester & OK & 3.2 & 26.3 & 3 & 84 & 3 \\
\hline 746 & Ponca City & OK & 4.1 & 24.3 & 3 & 84 & 3 \\
\hline 747 & Durant & OK & 3.2 & 26.1 & 3 & 83 & 3 \\
\hline 748 & Seminole & OK & 3.5 & 27.4 & 3 & 84 & 3 \\
\hline 749 & Poteau & $\mathrm{OK}$ & 3.1 & 25.3 & 3 & 84 & 3 \\
\hline 750 & Sherman & TX & 2.9 & 29.7 & 3 & 78 & 3 \\
\hline 751 & Corsicana & TX & 2.4 & 32.5 & 3 & 77 & 3 \\
\hline 752 & Dallas & TX & 2.4 & 36.7 & 3 & 83 & 3 \\
\hline 753 & Dallas & TX & 2.4 & 36.7 & 3 & 83 & 3 \\
\hline 754 & Greenville & TX & 2.8 & 27.7 & 3 & 74 & 3 \\
\hline 755 & Texarkana & TX & 2.9 & 23 & 3 & 77 & 3 \\
\hline 756 & Longview & TX & 2.3 & 28.7 & 3 & 72 & 3 \\
\hline 757 & Tyler & TX & 2 & 24.9 & 3 & 79 & 3 \\
\hline 758 & Palestine & TX & 1.9 & 28.5 & 3 & 75 & 2 \\
\hline 759 & Lufkin & TX & 1.9 & 30.4 & 3 & 74 & 3 \\
\hline 760 & Fort Worth & TX & 2.4 & 36.3 & 3 & 81 & 3 \\
\hline 761 & Fort Worth & TX & 2.4 & 36.3 & 3 & 81 & 3 \\
\hline 762 & Denton & TX & 2.7 & 31.5 & 3 & 78 & 3 \\
\hline
\end{tabular}




\begin{tabular}{|c|c|c|c|c|c|c|c|}
\hline Zip & Location & State & HDD & CDH & FER & ICF & IECC \\
\hline 763 & Wichita Falls & $\mathrm{TX}$ & 3 & 34.5 & 3 & 81 & 3 \\
\hline 764 & Stephenville & TX & 2.7 & 27.4 & 3 & 76 & 3 \\
\hline 765 & Temple & TX & 2.2 & 33.1 & 3 & 78 & 2 \\
\hline 766 & Hillsboro & TX & 2.1 & 33.4 & 3 & 80 & 2 \\
\hline 767 & Waco & $\mathrm{TX}$ & 2.2 & 36.7 & 3 & 80 & 2 \\
\hline 768 & Brownwood & TX & 2.5 & 32.4 & 3 & 76 & 3 \\
\hline 769 & San Angelo & TX & 2.4 & 32.7 & 3 & 77 & 3 \\
\hline 770 & Houston & TX & 1.5 & 30.5 & 3 & 86 & 2 \\
\hline 771 & Houston & TX & 1.5 & 30.5 & 3 & 86 & 2 \\
\hline 772 & Houston & TX & 1.5 & 30.5 & 3 & 86 & 2 \\
\hline 773 & Conroe & TX & 1.6 & 30.5 & 3 & 76 & 2 \\
\hline 774 & Bay City & TX & 1 & 33 & 3 & 79 & 2 \\
\hline 775 & Galveston & TX & 1 & 31.9 & 3 & 86 & 2 \\
\hline 776 & Port Arthor & TX & 1.4 & 31.7 & 3 & 86 & 2 \\
\hline 777 & Beaumont & TX & 1.5 & 31.7 & 3 & 86 & 2 \\
\hline 778 & College Station & TX & 1.6 & 33.2 & 3 & 83 & 2 \\
\hline 779 & Victoria & $\mathrm{TX}$ & 1.2 & 37.3 & 3 & 78 & 2 \\
\hline 780 & Laredo & TX & 0.9 & 52.6 & 3 & 75 & 2 \\
\hline 781 & Karnes City & TX & 1.4 & 36.2 & 3 & 81 & 2 \\
\hline 782 & San Antonio & TX & 1.6 & 36.2 & 3 & 81 & 2 \\
\hline 783 & Kingsville & TX & 1 & 42.9 & 3 & 76 & 2 \\
\hline 784 & Corpus Christi & TX & 1 & 42 & 3 & 76 & 2 \\
\hline 785 & Brownsville & TX & 0.6 & 42.5 & 3 & 75 & 2 \\
\hline 786 & Austin & TX & 1.5 & 35.2 & 3 & 77 & 2 \\
\hline 787 & Austin & TX & 1.5 & 35.2 & 3 & 77 & 2 \\
\hline 788 & Uvalde & TX & 1.5 & 37.1 & 3 & 69 & 2 \\
\hline 789 & Giddings & TX & 1.8 & 34.2 & 3 & 72 & 2 \\
\hline 790 & Borger & TX & 4 & 17.5 & 3 & 80 & 4 \\
\hline 791 & Amarillo & TX & 4.3 & 15.7 & 3 & 80 & 4 \\
\hline 792 & Childress & TX & 3.3 & 27.1 & 3 & 78 & 3 \\
\hline 793 & Lubbock & TX & 3.5 & 18.2 & 3 & 75 & 4 \\
\hline 794 & Lubbock & TX & 3.5 & 18.2 & 3 & 75 & 4 \\
\hline 795 & Snyder & TX & 3.1 & 25.8 & 3 & 79 & 3 \\
\hline 796 & Abilene & $\mathrm{TX}$ & 2.7 & 31.9 & 3 & 79 & 3 \\
\hline 797 & Midland & TX & 2.7 & 25 & 3 & 76 & 3 \\
\hline 798 & Alpine & TX & 2.5 & 16.1 & 3 & 81 & 3 \\
\hline 799 & El Paso & TX & 2.5 & 23 & 3 & 91 & 3 \\
\hline 800 & Denver & $\mathrm{CO}$ & 6.1 & 5.9 & 4 & 93 & 5 \\
\hline 801 & Parker & $\mathrm{CO}$ & 6.3 & 3.5 & 4 & 93 & 5 \\
\hline 802 & Denver & $\mathrm{CO}$ & 6.1 & 5.9 & 4 & 93 & 5 \\
\hline 803 & Boulder & $\mathrm{CO}$ & 5.7 & 7.7 & 4 & 93 & 5 \\
\hline 804 & Golden/Dillon & $\mathrm{CO}$ & 10.9 & 0 & 4 & 92 & 7 \\
\hline 805 & Longmont & $\mathrm{CO}$ & 6.4 & 3.8 & 4 & 89 & 5 \\
\hline 806 & Greeley & $\mathrm{CO}$ & 6 & 5.1 & 4 & 84 & 5 \\
\hline 807 & Fort Morgan & $\mathrm{CO}$ & 6.3 & 7.2 & 4 & 93 & 5 \\
\hline 808 & Florissant & $\mathrm{CO}$ & 9.4 & 0.1 & 4 & 95 & 7 \\
\hline 809 & Colorado Springs & $\mathrm{CO}$ & 6.5 & 3.7 & 4 & 95 & 5 \\
\hline 810 & Pueblo & $\mathrm{CO}$ & 5.6 & 11 & 4 & 93 & 5 \\
\hline
\end{tabular}




\begin{tabular}{|c|c|c|c|c|c|c|c|}
\hline Zip & Location & State & HDD & CDH & FER & ICF & IECC \\
\hline 811 & Alamosa & $\mathrm{CO}$ & 8.7 & 0 & 4 & 93 & 6 \\
\hline 812 & Salida & $\mathrm{CO}$ & 7.3 & 0 & 4 & 93 & 6 \\
\hline 813 & Durango & $\mathrm{CO}$ & 6.8 & 0.4 & 4 & 93 & 5 \\
\hline 814 & Montrose & $\mathrm{CO}$ & 6.3 & 3.6 & 4 & 93 & 5 \\
\hline 815 & Grand Junction & $\mathrm{CO}$ & 5.7 & 12.1 & 4 & 86 & 5 \\
\hline 816 & Glenwood Springs & $\mathrm{CO}$ & 6.7 & 2.1 & 4 & 92 & 5 \\
\hline 817 & ZIP NOT ASSIGNED & & 0 & 0 & 0 & 0 & 0 \\
\hline 818 & ZIP NOT ASSIGNED & & 0 & 0 & 0 & 0 & 0 \\
\hline 819 & ZIP_NOT_ASSIGNED & & 0 & 0 & 0 & 0 & 0 \\
\hline 820 & Cheyenne & WY & 7.4 & 2.1 & 4 & 83 & 6 \\
\hline 821 & Yellowstone Nat Pk & WY & 11.5 & 0.5 & 4 & 82 & 7 \\
\hline 822 & Wheatland & WY & 6.3 & 5.4 & 4 & 81 & 5 \\
\hline 823 & Rawlins & WY & 7.9 & 0.3 & 4 & 81 & 6 \\
\hline 824 & Thermopolis & WY & 8.3 & 5.2 & 4 & 80 & 6 \\
\hline 825 & Riverton & WY & 8.4 & 2.6 & 4 & 81 & 6 \\
\hline 826 & Casper & WY & 7.6 & 4.5 & 4 & 81 & 6 \\
\hline 827 & Gillette & WY & 8 & 4.3 & 4 & 80 & 6 \\
\hline 828 & Sheridan & WY & 7.7 & 4.5 & 4 & 83 & 6 \\
\hline 829 & Rock Springs & WY & 8.7 & 1 & 4 & 80 & 6 \\
\hline 830 & Jackson & WY & 9.5 & 0.1 & 4 & 80 & 7 \\
\hline 831 & Kemmerer & WY & 9.8 & 0.3 & 4 & 80 & 7 \\
\hline 832 & Pocatello & ID & 7.1 & 3.3 & 4 & 82 & 6 \\
\hline 833 & Twin Falls & ID & 6.7 & 2.8 & 4 & 73 & 5 \\
\hline 834 & Idaho Falls & ID & 7.9 & 1.4 & 4 & 75 & 6 \\
\hline 835 & Lewiston & ID & 5.2 & 7.9 & 4 & 114 & 5 \\
\hline 836 & Payette & ID & 5.6 & 6.8 & 4 & 86 & 5 \\
\hline 837 & Boise & ID & 5.7 & 8 & 4 & 86 & 5 \\
\hline 838 & Doeur D Alene & ID & 6.5 & 2.8 & 4 & 113 & 5 \\
\hline 839 & ZIP NOT ASSIGNED & & 0 & 0 & 0 & 0 & 0 \\
\hline 840 & Heber City & UT & 7 & 0.5 & 4 & 86 & 5 \\
\hline 841 & Salt Lake City & UT & 5.6 & 9.9 & 4 & 86 & 5 \\
\hline 842 & Ogden & UT & 5.9 & 9 & 4 & 84 & 5 \\
\hline 843 & Logan & UT & 6.7 & 5 & 4 & 85 & 5 \\
\hline 844 & Ogden & UT & 5.9 & 9 & 4 & 84 & 5 \\
\hline 845 & Blanding & UT & 5.5 & 4.2 & 4 & 81 & 5 \\
\hline 846 & Provo & UT & 5.3 & 9 & 4 & 86 & 5 \\
\hline 847 & Cedar City & UT & 6 & 5 & 4 & 86 & 5 \\
\hline 848 & ZIP NOT ASSIGNED & & 0 & 0 & 0 & 0 & 0 \\
\hline 849 & ZIP_NOT_ASSIGNED & & 0 & 0 & 0 & 0 & 0 \\
\hline 850 & Phoenix & $\mathrm{AZ}$ & 1.1 & 55 & 4 & 89 & 2 \\
\hline 851 & Not used & NU & 0 & 0 & 0 & 0 & 0 \\
\hline 852 & Casa Grande & $\mathrm{AZ}$ & 1.7 & 49 & 4 & 85 & 2 \\
\hline 853 & Buckeye & $\mathrm{AZ}$ & 1.5 & 55 & 4 & 89 & 2 \\
\hline 854 & ZIP NOT ASSIGNED & & 0 & 0 & 0 & 0 & 0 \\
\hline 855 & Globe & $\mathrm{AZ}$ & 3.1 & 24.6 & 4 & 84 & 4 \\
\hline 856 & Nogales & $\mathrm{AZ}$ & 2.9 & 9.9 & 4 & 88 & 2 \\
\hline 857 & Tucson & $\mathrm{AZ}$ & 1.6 & 36 & 4 & 88 & 2 \\
\hline 858 & ZIP NOT ASSIGNED & & 0 & 0 & 0 & 0 & 0 \\
\hline
\end{tabular}




\begin{tabular}{|c|c|c|c|c|c|c|c|}
\hline Zip & Location & State & HDD & CDH & FER & ICF & IECC \\
\hline 859 & Show Low & $\mathrm{AZ}$ & 4.9 & 4.6 & 4 & 85 & 5 \\
\hline 860 & Flagstaff & $\mathrm{AZ}$ & 7 & 0.4 & 4 & 86 & 5 \\
\hline 861 & ZIP NOT ASSIGNED & & 0 & 0 & 0 & 0 & 0 \\
\hline 862 & ZIP_NOT_ASSIGNED & & 0 & 0 & 0 & 0 & 0 \\
\hline 863 & Prescott & $\mathrm{AZ}$ & 4.8 & 3.8 & 4 & 82 & 4 \\
\hline 864 & Kingman & $\mathrm{AZ}$ & 3.3 & 21.6 & 4 & 84 & 3 \\
\hline 865 & Window Rock & $\mathrm{AZ}$ & 6.5 & 1.9 & 4 & 82 & 5 \\
\hline 866 & ZIP_NOT_ASSIGNED & & 0 & 0 & 0 & 0 & 0 \\
\hline 867 & ZIP_NOT_ASSIGNED & & 0 & 0 & 0 & 0 & 0 \\
\hline 868 & ZIP NOT ASSIGNED & & 0 & 0 & 0 & 0 & 0 \\
\hline 869 & ZIP_NOT_ASSIGNED & & 0 & 0 & 0 & 0 & 0 \\
\hline 870 & Albuquerque & NM & 4.3 & 11 & 4 & 88 & 4 \\
\hline 871 & Albuquerque & NM & 4.3 & 11 & 4 & 88 & 4 \\
\hline 872 & Albuquerque & NM & 4.3 & 11 & 4 & 88 & 4 \\
\hline 873 & Gallup & NM & 6.6 & 1.9 & 4 & 89 & 5 \\
\hline 874 & Farmington & NM & 5.5 & 5 & 4 & 88 & 5 \\
\hline 875 & Santa Fe & NM & 6.1 & 1.2 & 4 & 88 & 5 \\
\hline 876 & ZIP_NOT_ASSIGNED & & 0 & 0 & 0 & 0 & 0 \\
\hline 877 & Las Vegas & NM & 5.8 & 1.1 & 4 & 88 & 5 \\
\hline 878 & Socorro & NM & 4 & 11 & 4 & 88 & 4 \\
\hline 879 & Truth or Conseq & NM & 3.3 & 14.6 & 4 & 77 & 4 \\
\hline 880 & Lordsburg & NM & 3.3 & 19.2 & 4 & 77 & 3 \\
\hline 881 & Clovis & NM & 4.1 & 10 & 4 & 88 & 4 \\
\hline 882 & Roswell & NM & 3.3 & 20 & 4 & 88 & 3 \\
\hline 883 & Carrizozo & NM & 4.3 & 7.2 & 4 & 89 & 3 \\
\hline 884 & Tucumcari & NM & 3.9 & 15 & 4 & 88 & 4 \\
\hline 885 & El Paso & $\mathrm{TX}$ & 2.5 & 23 & 3 & 81 & 3 \\
\hline 886 & ZIP NOT ASSIGNED & & 0 & 0 & 0 & 0 & 0 \\
\hline 887 & ZIP_NOT_ASSIGNED & & 0 & 0 & 0 & 0 & 0 \\
\hline 888 & ZIP_NOT_ASSIGNED & & 0 & 0 & 0 & 0 & 0 \\
\hline 889 & Las Vegas & NV & 2.2 & 43 & 4 & 102 & 3 \\
\hline 890 & Tonopah & NV & 5.5 & 5.9 & 4 & 102 & 5 \\
\hline 891 & Las Vegas & NV & 2.2 & 43 & 4 & 102 & 3 \\
\hline 892 & ZIP_NOT_ASSIGNED & & 0 & 0 & 0 & 0 & 0 \\
\hline 893 & Ely & NV & 7.6 & 0.7 & 4 & 89 & 5 \\
\hline 894 & Winnemucca & $\mathrm{NV}$ & 6.3 & 4.3 & 4 & 94 & 5 \\
\hline 895 & Reno & NV & 5.6 & 2.2 & 4 & 94 & 5 \\
\hline 896 & Not used & NU & 0 & 0 & 0 & 0 & 0 \\
\hline 897 & Carson City & NV & 5.7 & 2 & 4 & 94 & 5 \\
\hline 898 & Elko & NV & 7.2 & 3.8 & 4 & 91 & 5 \\
\hline 899 & ZIP_NOT_ASSIGNED & & 0 & 0 & 0 & 0 & 0 \\
\hline 900 & Los Angeles & $\mathrm{CA}$ & 0.9 & 10.6 & 4 & 107 & 3 \\
\hline 901 & Los Angeles & $\mathrm{CA}$ & 0.9 & 10.6 & 4 & 107 & 3 \\
\hline 902 & Los Angeles & $\mathrm{CA}$ & 0.9 & 10.6 & 4 & 107 & 3 \\
\hline 903 & Inglewood & $\mathrm{CA}$ & 1.3 & 4.3 & 4 & 106 & 3 \\
\hline 904 & Santa Monica & $\mathrm{CA}$ & 1.8 & 1.9 & 4 & 106 & 3 \\
\hline 905 & Torrance & $\mathrm{CA}$ & 1.5 & 3.9 & 4 & 106 & 3 \\
\hline 906 & Montebello & $\mathrm{CA}$ & 0.9 & 10.2 & 4 & 106 & 3 \\
\hline
\end{tabular}




\begin{tabular}{|c|c|c|c|c|c|c|c|}
\hline Zip & Location & State & HDD & CDH & FER & ICF & IECC \\
\hline 907 & San Pedro & $\mathrm{CA}$ & 1.3 & 7.8 & 4 & 106 & 3 \\
\hline 908 & Long Beach & $\mathrm{CA}$ & 1.3 & 7.8 & 4 & 106 & 3 \\
\hline 909 & ZIP NOT ASSIGNED & & 0 & 0 & 0 & 0 & 0 \\
\hline 910 & Pasadena & $\mathrm{CA}$ & 1.4 & 11 & 4 & 104 & 3 \\
\hline 911 & Pasadena & $\mathrm{CA}$ & 1.4 & 11 & 4 & 104 & 3 \\
\hline 912 & Glendale & $\mathrm{CA}$ & 1.6 & 11.4 & 4 & 104 & 3 \\
\hline 913 & Canoga Park & $\mathrm{CA}$ & 1.8 & 13 & 4 & 105 & 3 \\
\hline 914 & Van Nuys & $\mathrm{CA}$ & 1.8 & 11.4 & 4 & 105 & 3 \\
\hline 915 & Burbank & $\mathrm{CA}$ & 1.6 & 11.4 & 4 & 105 & 3 \\
\hline 916 & North Hollywood & $\mathrm{CA}$ & 1.6 & 11.4 & 4 & 105 & 3 \\
\hline 917 & Pomona & $\mathrm{CA}$ & 1.7 & 10.2 & 4 & 105 & 3 \\
\hline 918 & Alhambra & $\mathrm{CA}$ & 1.4 & 11 & 4 & 105 & 3 \\
\hline 919 & Alpine & $\mathrm{CA}$ & 1.9 & 6.6 & 4 & 107 & 3 \\
\hline 920 & Escondido & $\mathrm{CA}$ & 1.5 & 6.6 & 4 & 107 & 3 \\
\hline 921 & San Diego & $\mathrm{CA}$ & 1.1 & 4.6 & 4 & 107 & 3 \\
\hline 922 & Palm Springs & $\mathrm{CA}$ & 0.9 & 54 & 4 & 107 & 2 \\
\hline 923 & Redlands & $\mathrm{CA}$ & 1.9 & 16.9 & 4 & 107 & 3 \\
\hline 924 & San Bernadino & $\mathrm{CA}$ & 1.6 & 17.8 & 4 & 107 & 3 \\
\hline 925 & Riverside & $\mathrm{CA}$ & 1.5 & 14.2 & 4 & 107 & 3 \\
\hline 926 & San Clemente & $\mathrm{CA}$ & 1.8 & 7.8 & 4 & 107 & 3 \\
\hline 927 & Santa Ana & $\mathrm{CA}$ & 1.2 & 6.9 & 4 & 107 & 3 \\
\hline 928 & Anaheim & $\mathrm{CA}$ & 1.3 & 6.9 & 4 & 107 & 3 \\
\hline 929 & ZIP_NOT_ASSIGNED & & 0 & 0 & 0 & 0 & 0 \\
\hline 930 & Oxnard & $\mathrm{CA}$ & 1.9 & 1.2 & 4 & 108 & 3 \\
\hline 931 & Santa Barbara & $\mathrm{CA}$ & 1.9 & 0.9 & 4 & 105 & 3 \\
\hline 932 & Visalia & $\mathrm{CA}$ & 2.6 & 19 & 4 & 104 & 3 \\
\hline 933 & Bakersfield & $\mathrm{CA}$ & 2.1 & 30 & 4 & 104 & 3 \\
\hline 934 & San Luis Obispo & $\mathrm{CA}$ & 2.1 & 1.1 & 4 & 105 & 3 \\
\hline 935 & Lancaster & $\mathrm{CA}$ & 3.2 & 21 & 4 & 104 & 4 \\
\hline 936 & Madera & $\mathrm{CA}$ & 2.7 & 17.9 & 4 & 102 & 3 \\
\hline 937 & Fresno & $\mathrm{CA}$ & 2.4 & 19.4 & 4 & 102 & 3 \\
\hline 938 & Fresno & $\mathrm{CA}$ & 2.4 & 19.4 & 4 & 102 & 3 \\
\hline 939 & Monterey & $\mathrm{CA}$ & 3.1 & 0.1 & 4 & 109 & 3 \\
\hline 940 & Half Moon Bay & $\mathrm{CA}$ & 3.7 & 0.1 & 4 & 121 & 3 \\
\hline 941 & San Francisco & $\mathrm{CA}$ & 2.9 & 0.2 & 4 & 121 & 3 \\
\hline 942 & Sacramento & $\mathrm{CA}$ & 2.7 & 7.8 & 4 & 113 & 3 \\
\hline 943 & Palo Alto & $\mathrm{CA}$ & 2.6 & 0.7 & 4 & 118 & 3 \\
\hline 944 & San Mateo & $\mathrm{CA}$ & 2.6 & 1.2 & 4 & 119 & 3 \\
\hline 945 & Napa & $\mathrm{CA}$ & 2.7 & 1.4 & 4 & 119 & 3 \\
\hline 946 & Oakland & $\mathrm{CA}$ & 2.4 & 0.4 & 4 & 118 & 3 \\
\hline 947 & Berkeley & $\mathrm{CA}$ & 2.9 & 0.3 & 4 & 119 & 3 \\
\hline 948 & Richmond & $\mathrm{CA}$ & 2.9 & 0.4 & 4 & 118 & 3 \\
\hline 949 & San Rafael & $\mathrm{CA}$ & 2.6 & 1.9 & 4 & 120 & 3 \\
\hline 950 & Gilroy & $\mathrm{CA}$ & 2.3 & 0.1 & 4 & 110 & 3 \\
\hline 951 & San Jose & $\mathrm{CA}$ & 2.2 & 1.4 & 4 & 113 & 3 \\
\hline 952 & Stockton & $\mathrm{CA}$ & 2.6 & 13 & 4 & 109 & 4 \\
\hline 953 & Merced & $\mathrm{CA}$ & 2.6 & 14 & 4 & 109 & 4 \\
\hline 954 & Santa Rosa & $\mathrm{CA}$ & 2.7 & 1.2 & 4 & 113 & 3 \\
\hline
\end{tabular}




\begin{tabular}{|c|c|c|c|c|c|c|c|}
\hline Zip & Location & State & HDD & CDH & FER & ICF & IECC \\
\hline 955 & Eureka & $\mathrm{CA}$ & 4.4 & 0 & 4 & 109 & 4 \\
\hline 956 & Placerville & $\mathrm{CA}$ & 3.3 & 7.8 & 4 & 113 & 4 \\
\hline 957 & Blue Canyon & $\mathrm{CA}$ & 5.5 & 4.1 & 4 & 113 & 5 \\
\hline 958 & Sacramento & $\mathrm{CA}$ & 2.7 & 12 & 4 & 113 & 3 \\
\hline 959 & Marysville & $\mathrm{CA}$ & 2.5 & 15 & 4 & 109 & 3 \\
\hline 960 & Redding & $\mathrm{CA}$ & 3 & 28 & 4 & 109 & 3 \\
\hline 961 & Susanville & $\mathrm{CA}$ & 6.2 & 2.2 & 4 & 108 & 5 \\
\hline 962 & ZIP_NOT_ASSIGNED & & 0 & 0 & 0 & 0 & 0 \\
\hline 963 & ZIP_NOT_ASSIGNED & & 0 & 0 & 0 & 0 & 0 \\
\hline 964 & ZIP NOT ASSIGNED & & 0 & 0 & 0 & 0 & 0 \\
\hline 965 & ZIP NOT ASSIGNED & & 0 & 0 & 0 & 0 & 0 \\
\hline 966 & ZIP NOT ASSIGNED & & 0 & 0 & 0 & 0 & 0 \\
\hline 967 & Lihui & $\mathrm{HI}$ & 0 & 25.6 & 4 & 114 & 1 \\
\hline 968 & Honolulu & $\mathrm{HI}$ & 0 & 36.2 & 4 & 116 & 1 \\
\hline 969 & Guam & GU & 0 & 53.2 & 4 & 93 & 1 \\
\hline 970 & Hood River & OR & 5.5 & 1.5 & 4 & 103 & 5 \\
\hline 971 & Seaside & OR & 4.5 & 0.1 & 4 & 103 & 4 \\
\hline 972 & Portland & OR & 4.4 & 1.9 & 4 & 103 & 4 \\
\hline 973 & Salem & OR & 4.8 & 1 & 4 & 102 & 4 \\
\hline 974 & Eugene & OR & 4.8 & 1.3 & 4 & 101 & 4 \\
\hline 975 & Medford & OR & 4.5 & 6.2 & 4 & 101 & 4 \\
\hline 976 & Klamath Falls & OR & 6.9 & 2.4 & 4 & 101 & 5 \\
\hline 977 & Bend & OR & 7 & 0.6 & 4 & 102 & 5 \\
\hline 978 & Pendleton & OR & 5.3 & 8.1 & 4 & 96 & 5 \\
\hline 979 & Ontario & OR & 6.1 & 10 & 4 & 95 & 5 \\
\hline 980 & Kent & WA & 4.6 & 0.5 & 4 & 102 & 4 \\
\hline 981 & Seattle & WA & 4.8 & 1 & 4 & 102 & 4 \\
\hline 982 & Everett & WA & 5.2 & 0.2 & 4 & 101 & 4 \\
\hline 983 & Port Angeles & WA & 5.6 & 0.2 & 4 & 101 & 4 \\
\hline 984 & Tacoma & WA & 4.7 & 0.5 & 4 & 101 & 4 \\
\hline 985 & Olympia & WA & 5.5 & 0.3 & 4 & 100 & 4 \\
\hline 986 & Vancouver & WA & 5 & 1.7 & 4 & 99 & 4 \\
\hline 987 & Not used & NU & 0 & 0 & 0 & 0 & 0 \\
\hline 988 & Wenatchee & WA & 6 & 7.6 & 4 & 93 & 5 \\
\hline 989 & Yakima & WA & 6.1 & 4.1 & 4 & 93 & 5 \\
\hline 990 & Spokane & WA & 6.8 & 3.5 & 4 & 117 & 5 \\
\hline 991 & Newport & WA & 7.2 & 1 & 4 & 117 & 6 \\
\hline 992 & Spokane & WA & 6.8 & 3.5 & 4 & 117 & 5 \\
\hline 993 & Richland & WA & 5.1 & 9.8 & 4 & 118 & 5 \\
\hline 994 & Clarkston & WA & 5.2 & 8 & 4 & 116 & 5 \\
\hline 995 & Anchorage & $\mathrm{AK}$ & 10.5 & 0 & 4 & 150 & 7 \\
\hline 996 & McGrath & $\mathrm{AK}$ & 13.9 & 0.4 & 4 & 150 & 8 \\
\hline 997 & Fairbanks & $\mathrm{AK}$ & 14 & 0 & 4 & 150 & 8 \\
\hline 998 & Juneau & $\mathrm{AK}$ & 8.6 & 0 & 4 & 149 & 7 \\
\hline 999 & Ketchikan & $\mathrm{AK}$ & 7.2 & 0 & 4 & 150 & 7 \\
\hline
\end{tabular}


APPENDIX C TEXT OF THE 2008 DOE INSULATION FACT SHEET 
DOE/CE-0180

\section{8}

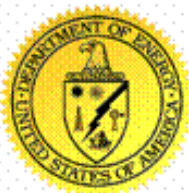

Department of Energy

Assistant Secretary

Energy Efficiency and Renewable

$$
\text { Energy }
$$

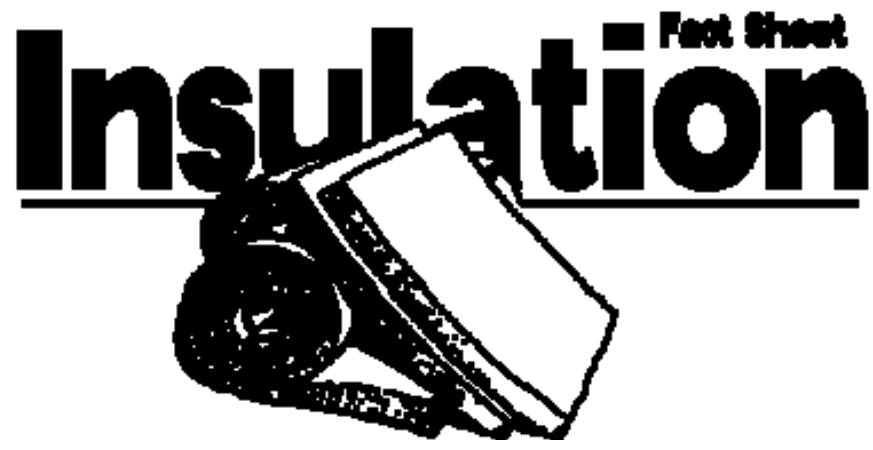

\section{Contents:}

\section{Introduction}

- Why Insulate Your House?

- How Insulation Works

Which Kind of Insulation is Best? Page 3

- What Is an R-Value?

- Reading the Label

- Insulation Product Types

Insulating a New House

Page 8

- Where and How Much?

- Air Sealing

- Moisture Control and Ventilation

- Installation Issues

o Precautions

o Attics

o Walls

- Design Options

o Crawlspaces and Slabs

o Advanced Wall Framing

o Metal Framing

o Insulating Concrete Forms

o Massive Walls

o Structural Insulated Panels

o External Insulation Finish System

o Attic Ventilation or a

Cathedralized Attic
Adding Insulation to an Existing House Page 20

- Where and How Much

- How Much Insulation Do I Already Have?

- Air Sealing

- Moisture Control and Ventilation

- Insulation Installation, the Retrofit Challenge

o Precautions

o Attics

o Walls

o Basement Walls

o Floors and Crawlspaces

Resources and Links

Page 30

About This Fact Sheet

Page 34 


\section{Why Insulate Your House?}

\section{Introduction}

Heating and cooling account for 50 to $70 \%$ of the energy used in the average American home. Inadequate insulation and air leakage are leading causes of energy waste in most homes. Insulation:

- $\quad$ saves money and our nation's limited energy resources

- makes your house more comfortable by helping to maintain a uniform temperature throughout the house, and

- makes walls, ceilings, and floors warmer in the winter and cooler in the summer.

The amount of energy you conserve will depend on several factors: your local climate; the size, shape, and construction of your house; the living habits of your family; the type and efficiency of the heating and cooling systems; and the fuel you use. Once the energy savings have paid for the installation cost, energy conserved is money saved - and saving energy will be even more important as utility rates go up.

This fact sheet will help you to understand how insulation works, what different types of insulation are available, and how much insulation makes sense for your climate. There are many other things you can do to conserve energy in your home as well. The Department of Energy offers many web sites to help you save energy by sealing air leaks, selecting more energy-efficient appliances, etc.

\section{How Insulation Works}

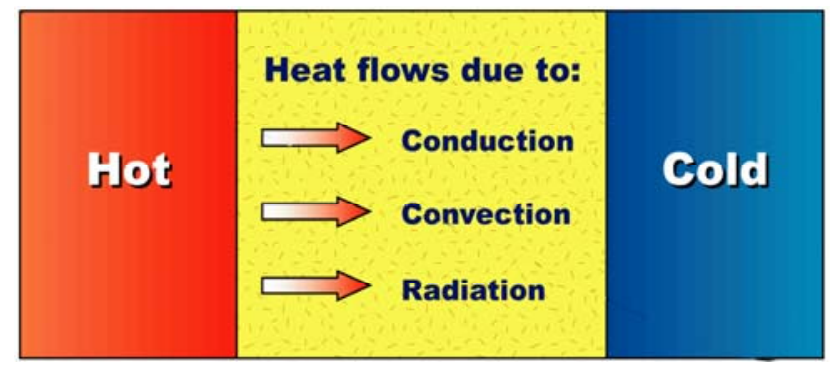

Heat flows naturally from a warmer to a cooler space. In winter, the heat moves directly from all heated living spaces to the outdoors and to adjacent unheated attics, garages, and basements - wherever there is a difference in temperature. During the summer, heat moves from outdoors to the house interior. To maintain comfort, the heat lost in winter must be replaced by your heating system and the heat gained in summer must be removed by your air conditioner. Insulating ceilings, walls, and floors decreases the heating or cooling needed by providing an effective resistance to the flow of heat.
Batts, blankets, loose fill, and low-density foams all work by limiting air movement. (These products may be more familiarly called fiberglass, cellulose, polyicynene, and expanded polystyrene.) The still air is an effective insulator because it eliminates convection and has low conduction. Some foams, such as polyisocyanurate, polyurethane, and extruded polystyrene, are filled with special gases that provide additional resistance to heat flow.
The ability of insulation to limit air movement should not be confused with "air sealing". The insulation reduces air movement only within the space it occupies. It will not reduce air movement through other cracks between building parts. For example, controlling air movement within a wall cavity will not stop air that leaks between the foundation and the sill plate or between the wall joists and a window frame.

Reflective insulation works by reducing the amount of energy that travels in the form of radiation. Some forms of reflective insulation also divide a space up into small regions to reduce air movement, or convection, but not to the same extent as batts, blankets, loose-fill, and foam. 


\section{Which Kind Of Insulation Is Best?}

Based on our email, this is one of the most popular questions homeowners ask before buying insulation. The answer is that the 'best' type of insulation depends on:

- how much insulation is needed,

- the accessibility of the insulation location,

- the space available for the insulation,

- local availability and price of insulation, and

- other considerations unique to each purchaser.

Whenever you compare insulation products, it is critical that you base your comparison on equal Rvalues.

\section{What Is an R-Value?}

Insulation is rated in terms of thermal resistance, called R-value, which indicates the resistance to heat flow. The higher the $R$-value, the greater the insulating effectiveness. The R-value of thermal insulation depends on the type of material, its thickness, and its density. In calculating the R-value of a multi-layered installation, the R-values of the individual layers are added.

The effectiveness of an insulated ceiling, wall or floor depends on how and where the insulation is installed.

- Insulation which is compressed will not give you its full rated R-value. This can happen if you add denser insulation on top of lighter insulation in an attic. It also happens if you place batts rated for one thickness into a thinner cavity, such as placing $\mathrm{R}-19$ insulation rated for 61/4 inches into a $5 \frac{1}{2}$ inch wall cavity.

- Insulation placed between joists, rafters, and studs does not retard heat flow through those joists or studs. This heat flow is called thermal bridging. So, the overall R-value of a wall or ceiling will be somewhat different from the R-value of the insulation itself. That is why it is important that attic insulation cover the tops of the joists and that is also why we often recommend the use of insulative sheathing on walls. The short-circuiting through metal framing is much greater than that through wood-framed walls; sometimes the insulated metal wall's overall R-value can be as low as half the insulation's R-value. 


\section{Reading the Label}

No matter what kind of insulation you buy, check the information on the product label to make sure that the product is suitable for the intended application. To protect consumers, the Federal Trade Commission has very clear rules about the R-value label that must be placed on all residential insulation products, whether they are installed by professionals, or whether they are purchased at a local supply store. These labels include a clearly stated R-value and information about health, safety, and fire-hazard issues. Take time to read the label BEFORE installing the insulation. Insist that any contractor installing insulation provide the product labels from EACH package (which will also tell you how many packages were used). Many special products have been developed to give higher Rvalues with less thickness. On the other hand, some materials require a greater initial thickness to offset eventual settling or to ensure that you get the rated R-value under a range of temperature conditions.

\section{Insulation Product Types}

Some types of insulation require professional installation, and others you can install yourself. You should consider the several forms of insulation available, their R-values, and the thickness needed. The type of insulation you use will be determined by the nature of the spaces in the house that you plan to insulate. For example, since you cannot conveniently "pour" insulation into an overhead space, blankets, spray-foam, board products, or reflective systems are used between the joists of an unfinished basement ceiling. The most economical way to fill closed cavities in finished walls is with blown-in insulation applied with pneumatic equipment or with sprayed-in-place foam insulation.

The different forms of insulation can be used together. For example, you can add batt or roll insulation over loose-fill insulation, or vice-versa. Usually, material of higher density (weight per unit volume) should not be placed on top of lower density insulation that is easily compressed. Doing so will reduce the thickness of the material underneath and thereby lower its R-value. There is one exception to this general rule: When attic temperatures drop below $0^{\circ} \mathrm{F}$, some low-density, fiberglass, loose-fill insulation installations may allow air to circulate between the top of your ceiling and the attic, decreasing the effectiveness of the insulation. You can eliminate this air circulation by covering the low-density, loose-fill insulation with a blanket insulation product or with a higher density loose-fill insulation.

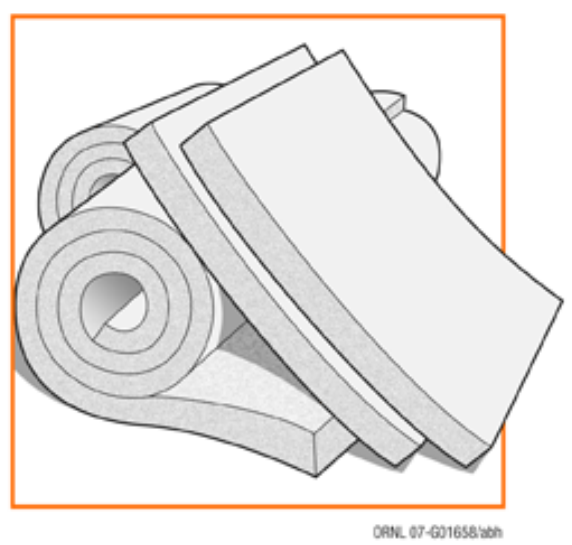

Insulation Fact Sheet - PDF version 2008
Blankets, in the form of batts or rolls, are flexible products made from mineral fibers, including fiberglass or rock wool. They are available in widths suited to standard spacings of wall studs and attic or floor joists. They must be hand-cut and trimmed to fit wherever the joist spacing is non-standard (such as near windows, doors, or corners), or where there are obstructions in the walls (such as wires, electrical outlet boxes, or pipes). Batts can be installed by homeowners or professionals. They are available with or without vapor-retarder facings. Batts with a special flame-resistant facing are available in various widths for basement walls where the insulation will be left exposed. 

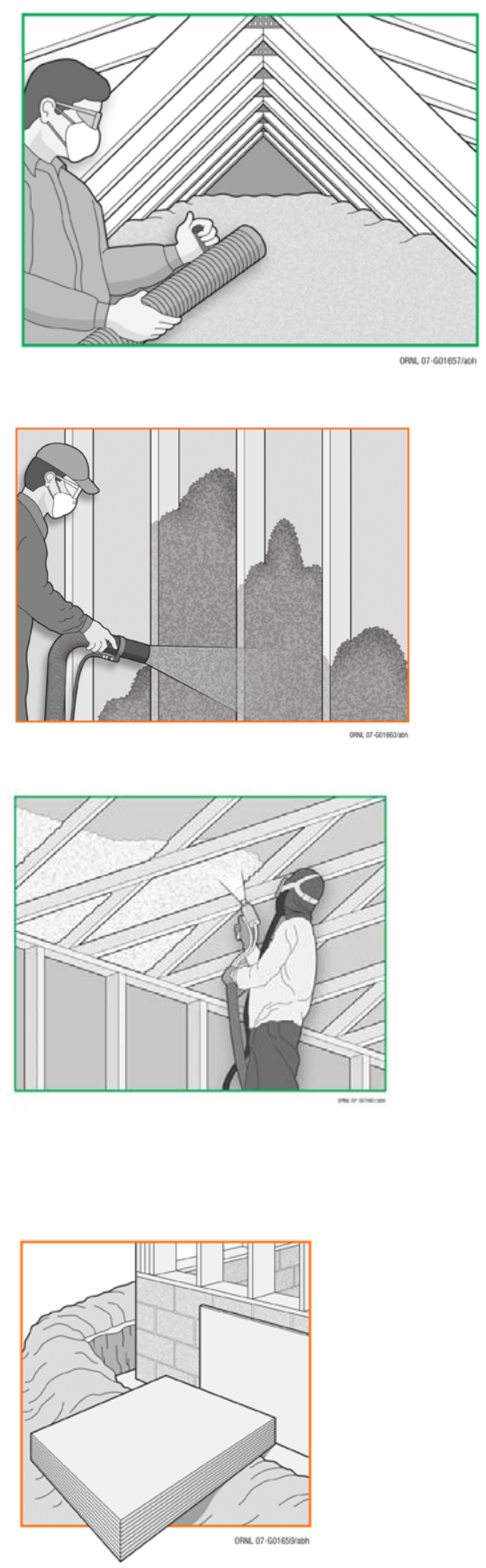

Blown-in loose-fill insulation includes cellulose, fiberglass, or rock wool in the form of loose fibers or fiber pellets that are blown using pneumatic equipment, usually by professional installers. This form of insulation can be used in wall cavities. It is also appropriate for unfinished attic floors, for irregularly shaped areas, and for filling in around obstructions.

In the open wall cavities of a new house, cellulose and fiberglass fibers can also be sprayed after mixing the fibers with an adhesive or foam to make them resistant to settling.

Foam insulation can be applied by a professional using special equipment to meter, mix, and spray the foam into place. Polyicynene is an open-celled foam. Polyisocyanurate and polyurethane are closed-cell foams. In general, open-celled foam allows water vapor to move through the material more easily than closedcell foam. However, open-celled foams usually have a lower R-value for a given thickness compared to closedcell foams. So, some of the closed-cell foams are able to provide a greater R-value where space is limited.

Rigid insulation is made from fibrous materials or plastic foams and is produced in board-like forms and molded pipe coverings. These provide full coverage with few heat loss paths and are often able to provide a greater R-value where space is limited. Such boards may be faced with a reflective foil that reduces heat flow when next to an air space. Rigid insulation is often used for foundations and as an insulative wall sheathing. 


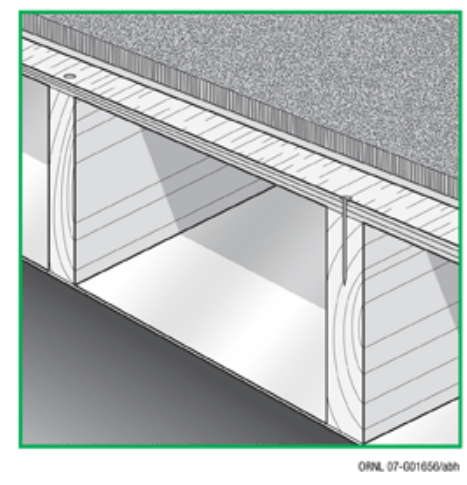

Reflective insulation systems are fabricated from aluminum foils with a variety of backings such as kraft paper, plastic film, polyethylene bubbles, or cardboard. The resistance to heat flow depends on the heat flow direction, and this type of insulation is most effective in reducing downward heat flow. Reflective systems are typically located between roof rafters, floor joists, or wall studs. If a single reflective surface is used alone and faces an open space, such as an attic, it is called a radiant barrier.

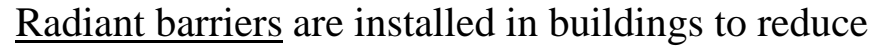
summer heat gain and winter heat loss. In new buildings, you can select foil-faced wood products for your roof sheathing (installed with the foil facing down into the attic) or other locations to provide the radiant barrier as an integral part of the structure. For existing buildings, the radiant barrier is typically fastened across the bottom of joists, as shown in this drawing. All radiant barriers must have a low emittance ( 0.1 or less) and high reflectance ( 0.9 or more). 


\section{Insulating a New House (Do It Right the First Time)}

If you are buying or building a new house, make sure that energy-saving features are included. The Federal Trade Commission (FTC) home insulation rule requires the seller of a new home to provide information on the type, thickness, and R-value of the insulation that will be installed in each part of the house in every sales contract. Many state or local building codes include minimum requirements for home insulation. Be sure that your new home or home addition meets these building codes. You may wish to install insulation beyond the minimum specified in such codes, especially if those minimum levels are below those recommended here. Also, Energy-Efficient Mortgages are available through both government-insured and conventional loan programs. These mortgages recognize that the homeowner's energy payments will be less for a more energy-efficient home, and therefore enable a buyer to borrow a larger sum to cover the up-front costs of improving the house's energy efficiency.

To keep initial selling prices competitive, many home builders offer standard (not optimal) levels of insulation, although additional insulation would be a good investment for the buyer. Builders participating in the Energy Star Program use third-party inspectors to not only ensure that the correct amount of insulation has been used, but also to ensure that it has been installed correctly.

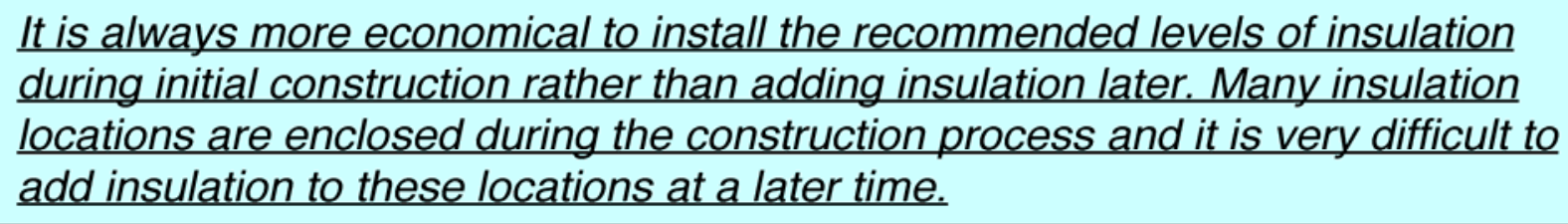

Now is also the time to make your home air tight. Special products and techniques are available to eliminate air leaks between the walls and floor and between the walls and ceiling. Encourage your builder to make all clearances around doors and windows as tight as possible and to properly caulk and seal all such joints.

\section{Where and How Much?}

Figure 1 shows which building spaces should be insulated. Discuss the house plans with your builder, and make sure each of these spaces is properly insulated to the R-values recommended here. Remember to buy the insulation based on this R-value, and to check the product label to determine the insulation's proper thickness, especially if you plan to install it in a confined space, such as in wall cavities and cathedral ceilings. 


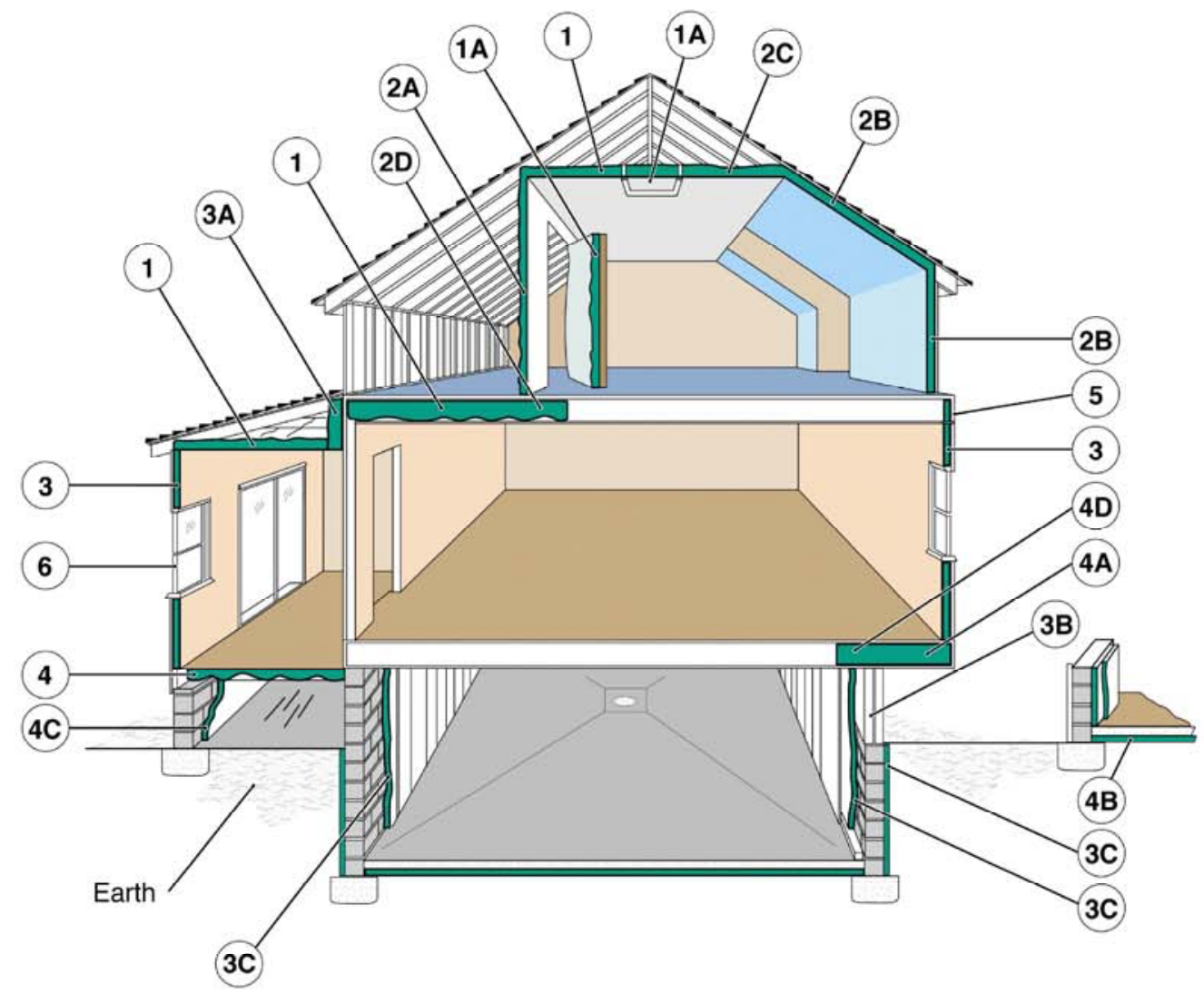

\section{Figure 1. Examples of Where to Insulate}

1. In unfinished attic spaces, insulate between and over the floor joists to seal off living 1A attic access doo

2. In finished attic rooms with or without dormer, insulate...
ee" walls:
erior walls and roof:
$2 \mathrm{C}$ ceilings with cold

2D extend insulation into joist space to reduce air flows.

3. All exterior walls, including.

$3 A$ walls between lining spaces and unheated garages, shed roots, or storage areas

$3 \mathrm{C}$ foundation walls in heated basements, full wall either interior or exterior.

4. Floors above cold spaces, such as vented crawl spaces and unheated garages. Also insulate. 4A any portion of the floor in a room that is cantilevered beyond the exterior wall below;

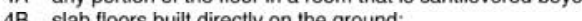

4C as an alternative to floor insulation, foundation walls of unvented crawl spaces: 4D extend insulation into joist space to reduce air flows.

5. Band joists

6. Replacement or storm windows and caulk and seal around all windows and doors. 


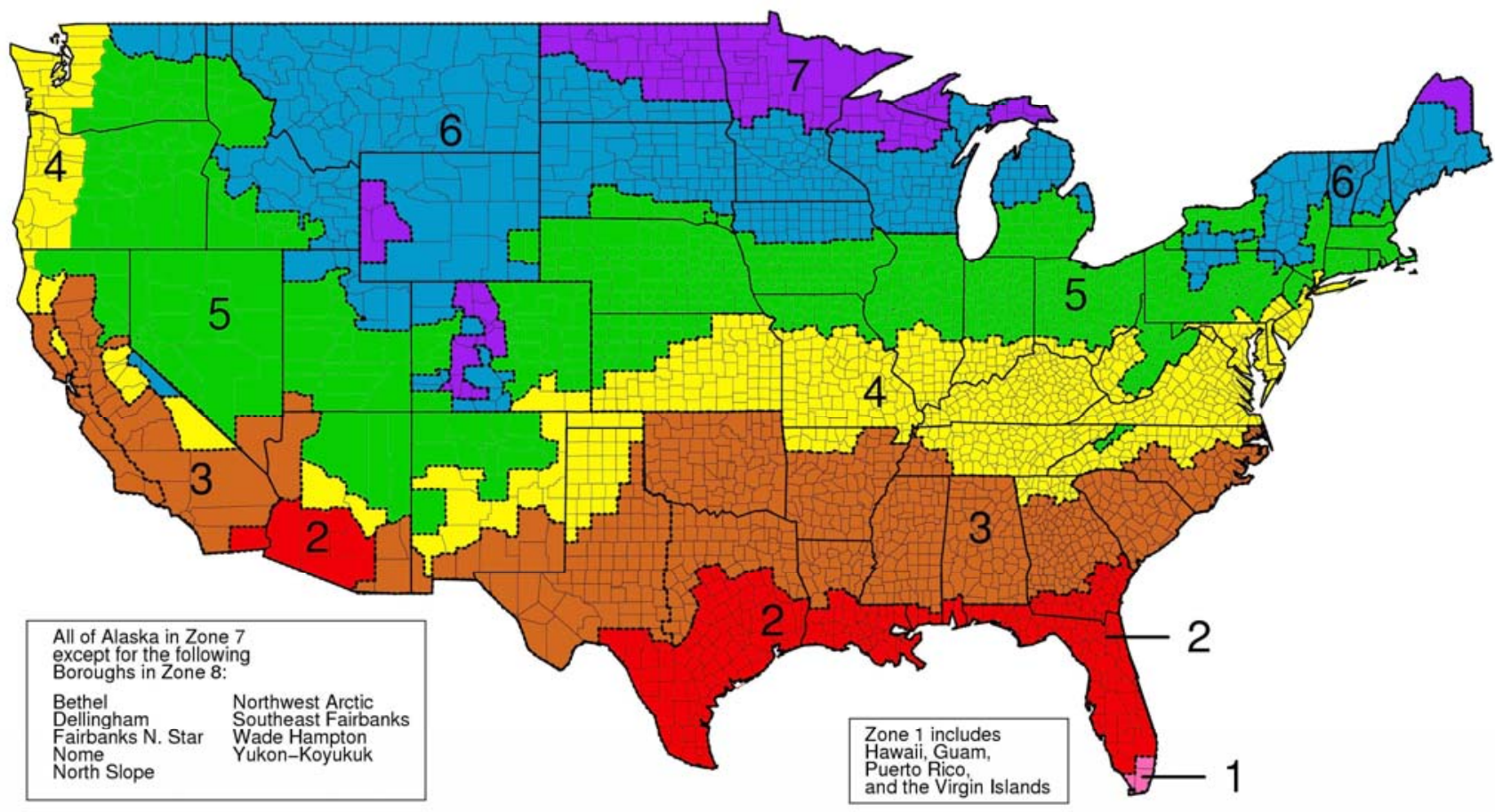

\begin{tabular}{|c|c|c|c|c|c|c|}
\hline \multirow{2}{*}{ Zone } & \multirow{2}{*}{ Heating system } & \multirow{2}{*}{ Attic } & \multirow{2}{*}{$\begin{array}{l}\text { Cathedral } \\
\text { Ceiling }\end{array}$} & \multicolumn{2}{|c|}{ Wall } & \multirow[b]{2}{*}{ Floor } \\
\hline & & & & Cavity & $\begin{array}{l}\text { Insulation } \\
\text { Sheathing }\end{array}$ & \\
\hline 1 & All & R30 to R49 & R22 to R38 & $\mathrm{R} 13$ to $\mathrm{R} 15$ & None & R13 \\
\hline 2 & $\begin{array}{l}\text { Gas, oil, heat pump } \\
\text { Electric furnace }\end{array}$ & R30 to R60 & R22 to R38 & $\mathrm{R} 13$ to $\mathrm{R} 15$ & None & $\begin{array}{c}\mathrm{R} 13 \\
\mathrm{R} 19-\mathrm{R} 25 \\
\end{array}$ \\
\hline 3 & $\begin{array}{l}\text { Gas, oil, heat pump } \\
\text { Electric furnace }\end{array}$ & $\mathrm{R} 30$ to $\mathrm{R} 60$ & $\mathrm{R} 22$ to $\mathrm{R} 38$ & $\mathrm{R} 13$ to $\mathrm{R} 15$ & $\begin{array}{c}\text { None } \\
\mathrm{R} 2.5 \text { to R5 } \\
\end{array}$ & R25 \\
\hline 4 & $\begin{array}{c}\text { Gas, oil, heat pump } \\
\text { Electric furnace }\end{array}$ & R38 to R60 & R30 to R38 & $\mathrm{R} 13$ to $\mathrm{R} 15$ & $\begin{array}{l}\mathrm{R} 2.5 \text { to } \mathrm{R} 6 \\
\mathrm{R} 5 \text { to } \mathrm{R} 6\end{array}$ & R25-R30 \\
\hline 5 & $\begin{array}{c}\text { Gas, oil, heat pump } \\
\text { Electric furnace }\end{array}$ & R38 to R60 & $\begin{array}{l}\mathrm{R} 30 \text { to } \mathrm{R} 38 \\
\mathrm{R} 30 \text { to } \mathrm{R} 60 \\
\end{array}$ & $\begin{array}{l}\mathrm{R} 13 \text { to } \mathrm{R} 15 \\
\mathrm{R} 13 \text { to } \mathrm{R} 21 \\
\end{array}$ & $\begin{array}{l}\mathrm{R} 2.5 \text { to } \mathrm{R} 6 \\
\mathrm{R} 5 \text { to } \mathrm{R} 6 \\
\end{array}$ & $\mathrm{R} 25-\mathrm{R} 30$ \\
\hline 6 & All & R49 to R60 & R30 to R60 & R13 to R21 & R5 to R6 & R25-R30 \\
\hline 7 & & R49 to R60 & R30 to R60 & $\mathrm{R} 13$ to $\mathrm{R} 21$ & R5 to R6 & $\mathrm{R} 25-\mathrm{R} 30$ \\
\hline 8 & All & R49 to R60 & R30 to R60 & R13 to R21 & R5 to R6 & R25-R30 \\
\hline
\end{tabular}


Figure 2 shows the Department of Energy's climate zones, along with a short summary of our insulation recommendations for new houses. These recommendations are based on comparing your future energy savings to the current cost of installing insulation. A range is shown for many locations for these reasons:

- $\quad$ Energy costs vary greatly over each zone.

- Installed insulation costs vary greatly over each zone.

- Heating and cooling equipment efficiency varies from house to house.

- $\quad$ Our best estimate of future energy costs may not be exactly correct.

So, how should you decide how much insulation to install?

The future energy savings, of course, depends upon how much energy costs in the future. Our best estimate is that fuel costs will rise at approximately the same rate as general inflation. If you think that fuel costs will increase more than that, you should install the greater amount of insulation shown. Looking at the map, if you think the energy costs in your area are greater than energy costs for other locations in same climate zone, you should install the greater amount of insulation.

We can also give you better guidance for your specific location and recommendations for other insulation locations in your home. The ZIP-Code calculator will actually let you enter your own insulation prices, energy costs, and heating and cooling system efficiencies. However, some personal computer security systems won't allow Java programs to run properly. The recommended R-values table can be helpful in those cases, because it will provide recommendations based on insulation and energy costs for your local area.

Both insulative sheathing and cavity insulation are specified for walls because it is important to use them together as a system. Any combination of sheathing and cavity insulation shown in Figure 2 will give you a similar life-cycle savings.

The band joists, or outside edges of the floor frames, should be insulated while the house is under construction. For most of the country, you should try to install $\mathrm{R}-30$ in this location. If you live in Climate Zone 1, R-19 is adequate. More detailed drawings and insulation techniques for the band joist are shown in the Wall Insulation Technology Fact Sheet.

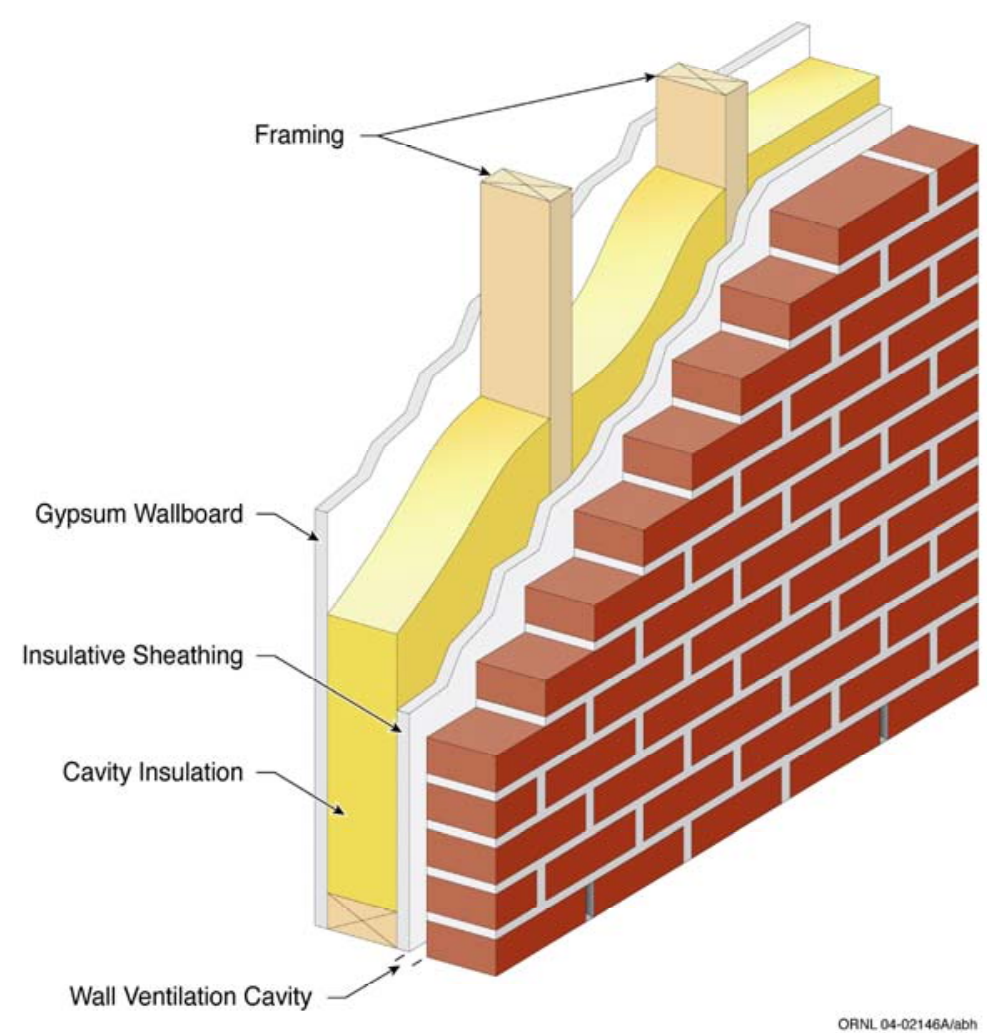


Foundation insulation options for new construction are broader than for existing homes. The builder may, for example, choose to insulate the exterior of a basement or crawlspace wall. You should discuss termite inspection and control options with your builder when choosing your foundation insulation method. Special sill plate (the joint between the top of the foundation and the bottom of the house frame) mineral fiber sealing products are designed to reduce air leaks if installed during the initial house construction. All flammable insulations or insulation facings must be covered or otherwise protected to meet fire codes. More information is given in the Basement Insulation Technology Fact Sheet.

If water lines and the ducts of your heating or air-conditioning system run through unheated or uncooled spaces, such as attic or crawlspaces, then the water lines and the ducts should be insulated. Make sure your contractor checks the ductwork for air leaks before installing the duct insulation. The contractor should then wrap the ducts with duct wrap insulation of R-6 with a vapor retarder facing on the outer side. All joints where sections of insulation meet should have overlapped facings and be tightly sealed with fiber glass tape; but avoid compressing the insulation, thus reducing its thickness and R-value.

Return air ducts are more likely to be located inside the heated portion of the house where they don't need to be insulated, but they should still be sealed off from air passageways that connect to unheated areas. Drywall-to-ductwork connections should be inspected because they are often poor (or nonexistent) and lead to unwanted air flows through wall cavities.

\section{Air Sealing}

Air sealing is important, not only because drafts are uncomfortable, but also because air leaks carry both moisture and energy, usually in the direction you don't want. For example, air leaks can carry hot humid outdoor air into your house in the summer, or can carry warm moist air from a bathroom into the attic in the winter.

Most homeowners are aware that air leaks into and out of their houses through small openings around doors and window frames and through fireplaces and chimneys. Air also enters the living space from other unheated parts of the house, such as attics, basements, or crawlspaces. The air travels through:

- $\quad$ any openings or cracks where two walls meet, where the wall meets the ceiling, or near interior door frames;

- gaps around electrical outlets, switch boxes, and recessed fixtures;

- $\quad$ gaps behind recessed cabinets, and furred or false ceilings such as kitchen or bathroom soffits;

- $\quad$ gaps around attic access hatches and pull-down stairs;

- $\quad$ behind bath tubs and shower stall units;

- through floor cavities of finished attics adjacent to unconditioned attic spaces;

- utility chaseways for ducts, etc., and

- plumbing and electrical wiring penetrations.

These leaks between the living space and other parts of the house are often much greater than the obvious leaks around windows and doors. Since many of these leakage paths are driven by the tendency for warm air to rise and cool air to fall, the attic is often the best place to stop them. It's important to stop these leaks before installing attic insulation because the insulation may hide them and make them less accessible. Usually, the attic insulation itself will not stop these leaks and you won't save as much as you expect because of the air flowing through or around the insulation. 
There are several fact sheets that will help you stop these air leaks:

- $\quad$ Air sealing

- $\quad$ Air Sealing for New Home Construction

- $\quad$ Air Sealing Technology Fact Sheet

When natural ventilation has been sharply reduced, as in a more energy-efficient house, it may be necessary to provide fresh air ventilation to avoid build-up of stale air and indoor air pollutants. Special air-to-air heat exchangers, or heat-recovery ventilators, are available for this purpose. It is also possible to incorporate a supply of fresh outside air into your heating and cooling system. This arrangement can be used to create a slightly higher pressure inside your home, which will prevent uncontrolled outside air infiltration into your home. For more details on this arrangement, see "Integration with forced-air heating and air conditioning systems" in the Whole-House Ventilation Systems Technology Fact Sheet.

\section{Moisture Control and Ventilation}

We talk about moisture control in an insulation fact sheet because wet insulation doesn't work well. Also, insulation is an important part of your building envelope system, and all parts of that system must work together to keep moisture from causing damage to the structure or being health hazards to the occupants. For example, mold and mildew grow in moist areas, causing allergic reactions and damaging buildings.

Moisture can enter your home during the construction process. The building materials can get wet during construction due to rain, dew, or by lying on the damp ground. Concrete walls and foundations release water steadily as they continue to cure during the first year after a home is built. During the house's first winter, this construction moisture may be released into the building at a rate of more than two gallons per day, and during the second winter at a slower rate of about one gallon per day. You may need to use dehumidifiers during this initial time period.

When Is Moisture a Problem?

Rain water can leak into your wall cavities if the windows are not properly flashed during installation. Also, when moist air touches a cold surface, some of the moisture may leave the air and condense, or become liquid. If moisture condenses inside a wall, or in your attic, you will not be able to see the water, but it can cause a number of problems.

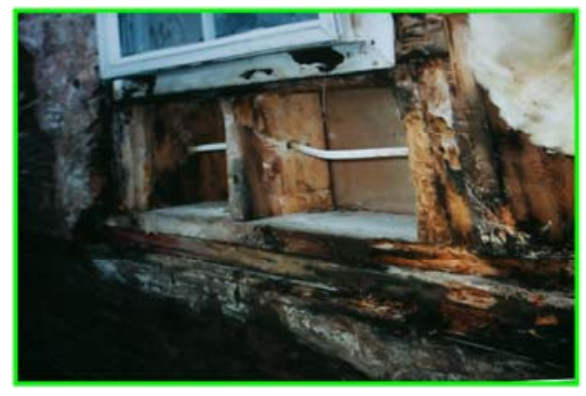

Four Things You Can Do to Avoid Moisture Problems:

- 1. Control liquid water. Rain coming through a wall, especially a basement or crawlspace wall, may be less apparent than a roof leak, especially if it is a relatively small leak and the water remains inside the wall cavity. Stop all rain-water paths into your home by:

o Use a Weather-Resistive Barrier. 
o Caulk around all your windows and doors.

o Direct all water coming off your roof away from your house by sloping the soil around your house so that water flows away from your house.

o Use wide overhangs to keep the rain away from your walls and windows.

o Use large gutters and gutter guards to help keep rain from dripping onto the ground near the house.

Be sure that the condensate from your air conditioner is properly drained away from your house. You should also be careful that watering systems for your lawn or flower beds do not spray water on the side of your house or saturate the ground near the house. It is also a good idea to check the caulking around your tub or shower to make sure that water is not leaking into your walls or floors.

- 2. Ventilate. You need to ventilate your home because you and your family generate moisture when you cook, shower, do laundry, and even when you breathe. More than $99 \%$ of the water used to water plants eventually enters the air. If you use an unvented natural gas, propane, or kerosene space heater, all the products of combustion, including water vapor, are exhausted directly into your living space. This water vapor can add up to 5 to 15 gallons of water per day to the air inside your home. If your clothes dryer is not vented to the outside, or if the outdoor vent is closed off or clogged, all that moisture will enter your living space. Just by breathing and perspiring, a typical family adds about 3 gallons of water per day to their indoor air. You especially need to vent your kitchen and bathrooms. Be sure that these vents go directly outside, and not to your attic, where the moisture can cause problems. Remember that a vent does not work unless you turn it on; so select quieter models that you are more likely to use. If your attic is ventilated, it is important that you never cover or block attic vents with insulation. Take care to prevent loose-fill insulation from clogging attic vents by using baffles or rafter vents. These baffles also serve to keep the outside air from penetrating into the insulation. When you think about venting to remove moisture, you should also think about where the replacement air will come from, and how it will get into your house. For more information about controlled ventilation, see the Whole-House Ventilation Systems Technology Fact Sheet.

- 3. Stop Air Leaks. It is very important to seal up all air-leakage paths between your living spaces and other parts of your building structure. Measurements have shown that air leaking into walls and attics carries significant amounts of moisture. Remember that if any air is leaking through electrical outlets or around plumbing connections into your wall cavities, moisture is carried along the same path. The same holds true for air moving through any leaks between your home and the attic, crawlspace, or garage. Even very small leaks in duct work can carry large amounts of moisture, because the airflow in your ducts is much greater than other airflows in your home. This is especially a problem if your ducts travel through a crawlspace or attic, so be sure to seal these ducts properly (and keep them sealed!). Return ducts are even more likely to be leaky, because they often involve joints between drywall and ductwork that may be poorly sealed, or even not sealed at all.

- 4. Plan a moisture escape path. Typical attic ventilation arrangements are one example of a planned escape path for moisture that has traveled from your home's interior into the attic space. Cold air almost always contains less water than hot air, so diffusion usually carries moisture from a warm place to a cold place. You can let moisture escape from a wall cavity to the dry outdoors during the winter, or to the dry indoors during the summer, by avoiding the use of vinyl wall coverings or low-perm paint. You can also use a dehumidifier to reduce 
moisture levels in your home, but it will increase your energy use and you must be sure to keep it clean to avoid mold growth. If you use a humidifier for comfort during the winter months, be sure that there are no closed-off rooms where the humidity level is too high.

Should you use vapor retarders?

Moisture can travel from the ground through the foundation and up into your walls, and through slab floors into your home. So your builder should always include a vapor retarder between the foundation and the walls. Should you include a vapor retarder in your wall? If so, where? If the outside air is colder and drier than the inside of a home, then moisture from inside the warm house will try to diffuse through the walls and ceiling toward the cold, dry outside air. If the outside air is hot and humid, then moisture from outside will try to diffuse through the walls toward the dry, air-conditioned inside air. We used to tell people to install vapor retarders to try and stop this moisture diffusion. But we have learned that if moisture moves both ways for significant parts of the year, you're better off not using a vapor retarder in the walls at all.

\section{Installation Issues}

Your builder will most likely hire a subcontractor to install the insulation in your new home. However, it is a good idea to educate yourself about proper installation methods because an improper installation can reduce your energy savings.

\section{INSULATION INSTALLATION PRECAUTIONS}

-Wear clothing adequate to protect against skin contact and irritation. A long-sleeved shirt with collar and cuffs buttoned, gloves, hat, glasses, and disposable dust respirator are advisable in all do-it-yourself insulation projects. Also, read the label and follow all the manufacturer's directions.

- Do not cover or hand-pack insulation around bare stove pipes, electrical fixtures, motors, or any heat-producing equipment such as recessed lighting fixtures. If you pack insulation around these heat-producing locations, the heat can build up, leading to fire. Electrical fire-safety codes prohibit the installation of thermal insulation within three inches of a recessed fixture enclosure, wiring compartment, or ballast, or above the fixture so that it will trap heat and prevent free circulation of air, unless the fixture is identified by label as suitable for insulation to be in direct contact with the fixture. THIS IS FOR FIRE SAFETY.

- Do not cover attic vents with insulation. Proper ventilation must be maintained to avoid overheating in summer and moisture build-up all year long. 
For blown-in loose fill insulation, each bag of insulating material used by the contractor should be marked with an R-value for the area to be covered. Although these figures may differ among manufacturers, the area figure will tell you the right number of bags to be used. Similarly, packages of other types of insulation should be identified by their R-value. It is important that you check that the proper amount is installed in your residence. Ask the contractor to attach vertical rulers to the joists prior to a loose-fill installation in your attic to help you see that the proper depth was installed. Also, the installer must provide a signed and dated statement describing the insulation installed, stating thickness, coverage area, R-value, and number of bags installed. In some areas, infrared thermography services are offered to help discover any gaps in the insulation.
In some houses, it is easier to get complete coverage of the attic floor with blown-in loose-fill insulation. Loose-fill insulation must be prevented from shifting into vents or from contacting heatproducing equipment (such as recessed lighting fixtures) by using baffles or retainers.

If batts or rolls are used, the first layer should be fit between the joists. The second layer should be placed perpendicular to the first because that will help to cover the tops of the joists themselves and reduce thermal bridging through the frame. Also, be sure to insulate the trap or access door. Although the area of the door is small, an uninsulated attic door will reduce energy savings substantially.

To be effective, Reflective Systems must be installed according to the manufacturer's instructions.

Radiant barriers may be installed in attics in several configurations. The radiant barrier is most often attached near the roof, to the bottom surface of the attic truss chords or to the rafter framing. A radiant barrier should never be placed on top of your insulation or on the attic floor because it will soon be covered with dust and will not work. A separate DOE fact sheet is available for radiant barriers to show which parts of the country are most likely to benefit from this type of system.

\section{Walls}

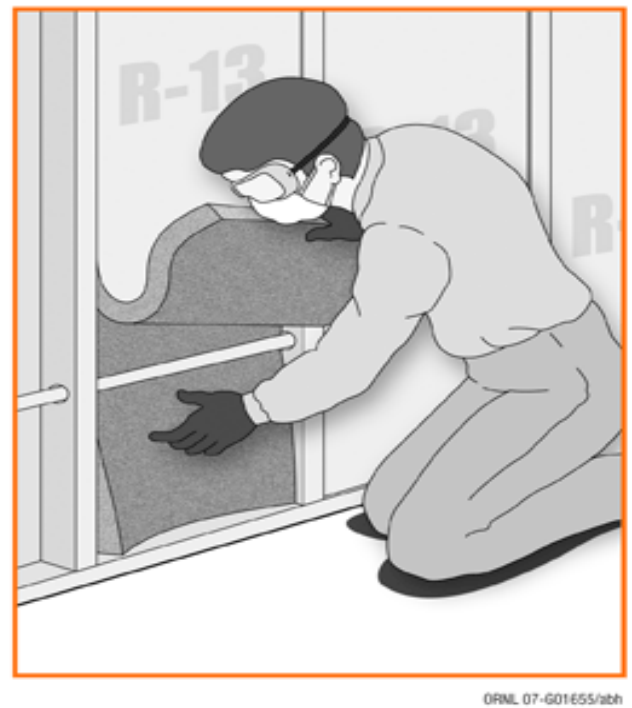

When batt insulation is installed, it is fit between the wood frame studs, according to the manufacturer's instructions. The batts must be carefully cut to fit around obstructions, such as window frames, pipes, wires, and electrical boxes with no gaps.

- Don't compress the insulation to fit behind pipes or wires. Instead cut to the middle of the batt's thickness so you have one flap under the pipe or wire and the other flap over the pipe or wire.

- $\quad$ One common mistake is to leave narrow places between close-spaced wall studs uninsulated. Even though these spaces may look like a very small part of the wall, small uninsulated areas can greatly reduce the insulation performance of the whole wall. Strips of insulation should be cut off and stuffed into such tight spaces by hand.

- The kraft paper or foil vapor retarder facings on many blanket insulation products must be covered with gypsum or interior paneling because of fire considerations. 
Blown-in cellulose or spray foam can be used to fill wall cavities. Both of these products can do a good job of filling in the space around wires and other obstructions and in filling any oddly-shaped areas. For cellulose, some form of netting is used to hold the cellulose in place until the drywall is installed. For spray-foam, it is important that the application be finished off neatly to avoid problems with the drywall installation.

Masonry walls should be insulated on the exterior surface.

\section{Design Options}

Design Option: Crawlspaces and Slabs

Many building codes presently require installation of crawlspace vents to provide ventilation with outside air, but there is no compelling technical basis for crawlspace ventilation requirements. If the crawlspace is vented, the floor should be insulated and any pipes or ducts in the crawlspace should be insulated as well. In some climates, pipes in vented crawlspaces must also be wrapped with heat trace wires to avoid frozen pipes. If the crawlspace is not vented, it is crucial that all of the crawlspace ground area be covered with a durable vapor retarder, such as heavy-weight polyethylene film. (This is also a good practice for vented crawlspaces.) For unvented crawlspaces, the insulation should be placed on the inside wall of the crawlspace instead of under the floor above. The insulation should also extend a few feet over the ground surface inside the crawlspace, lying on top of the durable vapor retarder. For the unvented arrangement, the air within the crawlspace is actually incorporated as a part of the conditioned space within the house, as if it were a basement. The Crawlspace Insulation Technology Fact Sheet gives more details about this design option. If you choose a slab foundation, be sure to follow the recommendations for insulation installation and moisture control found in the Slab Insulation Technology Fact Sheet.

Design Option: Advanced Wall Framing

Advanced wall framing techniques can be used that will reduce the energy losses through the walls with little or no additional costs. Details are given in the Wall Insulation Technology Fact Sheet.

Design Option: Metal Framing

Some new homes are built using metal frames instead of wood. Such frames are not susceptible to insect problems that can damage wood-framed structures. However, when you insulate a metal-framed building, it is important to recognize that much more heat flows through metal studs and joists than through pieces of wood. Because of this difference, placing insulation just between the wall studs, or just between attic or floor joists, doesn't work as well for metal-framed houses as it does for woodframed houses. If your walls have metal frames, you will need to place continuous insulative sheathing over the outside of the wall frame, between the metal framing pieces and your exterior siding in addition to insulating the space between the studs. (Note that this insulative sheathing cannot take the place of plywood or other seismic bracing.) If your attic has metal joists, you may want to place rigid foam insulation between the joists and the ceiling drywall and to cover the attic joists with insulation to the extent possible. 
Insulating concrete forms can be used to construct walls for new homes. These special concrete walls come in a variety of configurations and can provide additional thermal mass to your home to help reduce the effect of outdoor temperature swings.

Design Option: Massive Walls

The most common house type in this country is the light-construction frame house. Massive walls are less common, and include buildings made from concrete, concrete block, and log. These buildings will use less energy than wood-frame construction in many parts of the country because they can store heat from the daytime sun to provide needed heat at night, or can cool down at night to reduce airconditioning loads during the day. Research shows that such massive wall systems perform best if the insulation is located on the outside of the wall. See the ZipCode Calculator or the ZipCode table to find out how much insulation you should use.

Design Option: Structural Insulated Panels

Structural insulated panels can be used to construct a house. These panels sandwich plastic foam insulation between two layers of a wood or composite product, thus eliminating the need for structural wood framing members. These systems save energy in two ways, first by reducing the heat losses that would have gone through the wood frame, and second by reducing air leaks. Both of these changes represent significant energy savings. Just stopping the energy flowing through the wall framing could save $25 \%$ of the wall's energy losses.

Design Option: External Insulation Finish System

Some homes are built with an External Insulation Finish System that gives a stucco-like appearance. In the past, there were quality control problems where poor construction led to water leaks into the wall system, usually around window frames. Newer versions of the external insulation finish system have been designed to be more robust and to allow any water entering through a construction defect to drain harmlessly out of the wall. This system can be used to finish wood-framed, metal-framed, and masonry walls. It offers special advantages for the metal-framed and masonry walls because the continuous external insulation layer optimizes the thermal performance of both of those two wall systems.

\section{Design Option: Attic Ventilation or a Cathedralized Attic}

It is important that the house design, and construction, minimize the transfer of moisture from the living space to the attic. To handle any moisture that does migrate into the attic, traditional attic design calls for ventilation. Attics may be ventilated with a combination of soffit vents at eaves and continuous ridge vents. Attic vents may also be installed in gable faces. Many codes and standards require one square foot of unobstructed ventilation opening for each 300 square feet of attic floor area if a vapor retarder is included in the ceiling separating the attic from the living space. Twice as much ventilation is recommended if there is no vapor retarder. The net free area of a vent is smaller than its overall dimension because part of the vent opening is blocked by meshes or louvers. The openings should be equally distributed between the soffit and ridge vents or between each gable face. Never cover or block vents with insulation. Use baffles to prevent loose-fill insulation from clogging vents. 
During the house design phase, you can choose a cathedralized attic, which is not ventilated, unless attic ventilation is required by code in your area.

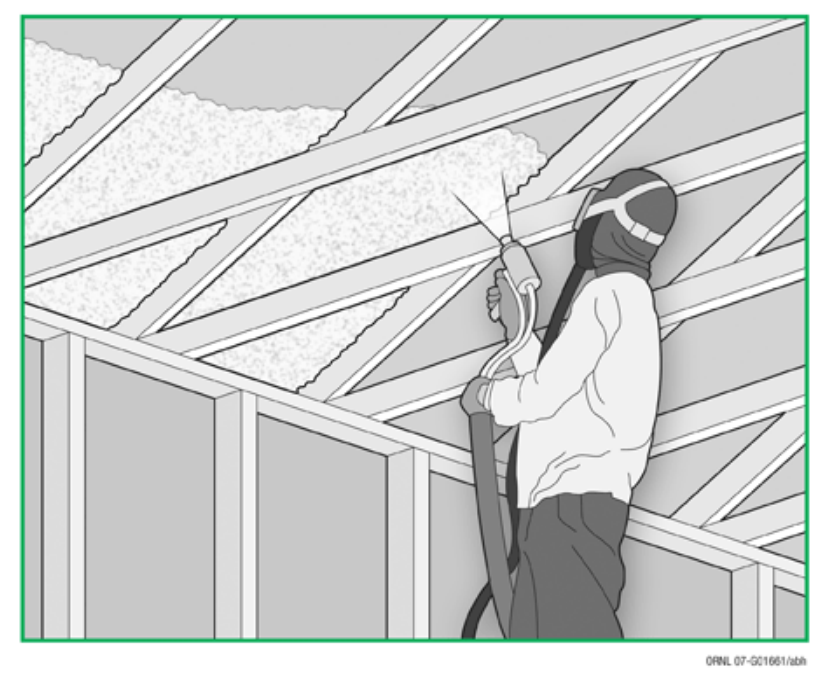

In a cathedralized attic, the insulation is placed on the underside of the roof instead of on the attic floor. (Think of a room with a cathedral ceiling, only this room is the attic and the ceiling is unfinished.) For this arrangement, the attic space is incorporated as a part of the conditioned space within the house. One advantage of this approach is that the attic will retain any energy lost by ductwork in the attic. Up to $25 \%$ of your heating and cooling energy can be wasted by leaky ductwork in a traditional attic.

A disadvantage of an unventilated attic is that the underside of the roof has a greater area than the attic floor. This greater area, and the downward-facing geometry, cause this option to be more costly than insulating the attic floor, so that usually the installed insulation R-value is less. The lower R-value and the greater area mean that more heat is lost through the cathedralized attic roof than would have been lost through the traditional attic floor. Also, a ventilated attic can reduce summer air conditioning loads relative to the cathedralized attic. The home owner must balance these two effects, reduced duct energy losses versus increased heating and cooling loads. Of course another option is to ventilate the attic, but locate the ductwork elsewhere within the conditioned part of the house, such as between floors in a multi-story building. 


\section{Adding Insulation to an Existing House (Smart Approaches)}

Does your home need more insulation? Unless your home was constructed with special attention to energy efficiency, adding insulation will probably reduce your utility bills. Much of the existing housing stock in the United States was not insulated to the levels used today. Older homes are likely to use more energy than newer homes, leading to higher heating and air-conditioning bills.

\section{Where and How Much}

Adding more insulation where you already have some, such as in an attic, will save energy. You can save even greater amounts of energy if you install insulation into places in your home that have never been insulated. Figure 1 shows which building spaces should be insulated. These might include an uninsulated floor over a garage or crawlspace, or a wall that separates a room from the attic. Figure 3 can give you general guidance regarding the appropriate amount of insulation you should add to your home, and the rest of this page will provide more specific information.

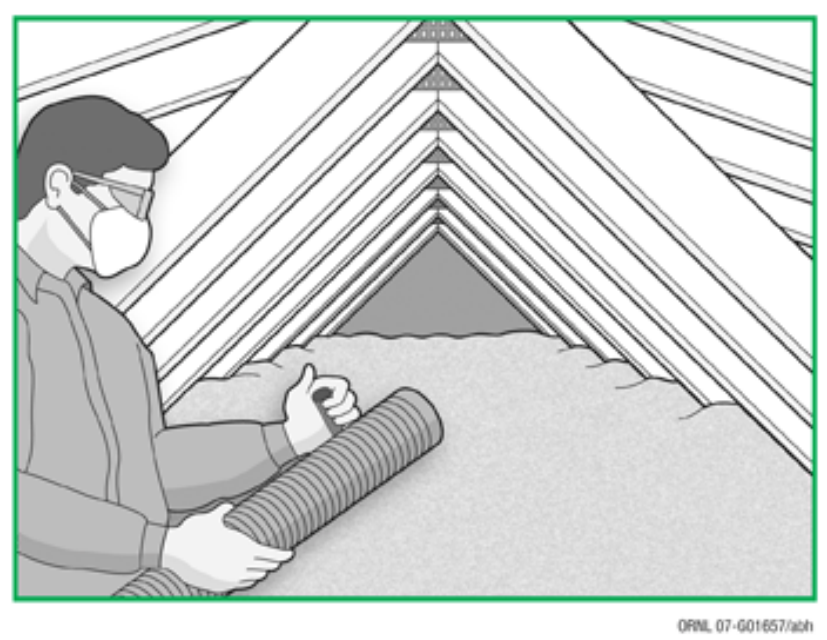

A qualified home energy auditor will include an insulation check as a routine part of an energy audit. For information about home energy audits, call your local utility company. State energy offices are another valuable resource for information. An energy audit of your house will identify the amount of insulation you have and need, and will likely recommend other improvements as well. If you don't have someone inspect your home, you'll need to find out how much insulation you already have.

After you find out how much you have, you can use the ZipCode tool to find out how much you should add. This recommendation balances future utility bill savings against the current cost of installing insulation. So the amount of insulation you need depends on your climate and heating fuel (gas, oil, electricity), and whether or not you have an air conditioner. The program is called the ZipCode because it includes weather and cost information for local regions defined by the first three digits of each postal service zip code. The program also allows you to define your own local costs and to input certain facts about your house to improve the accuracy of the recommendations. However, some personal computer security systems won't allow Java programs to run properly. The recommended Rvalues table can be helpful in those cases, because it will provide recommendations based on insulation and energy costs for your local area. 
Figure 3. Insulation Recommendations for Existing Wood-Framed Houses

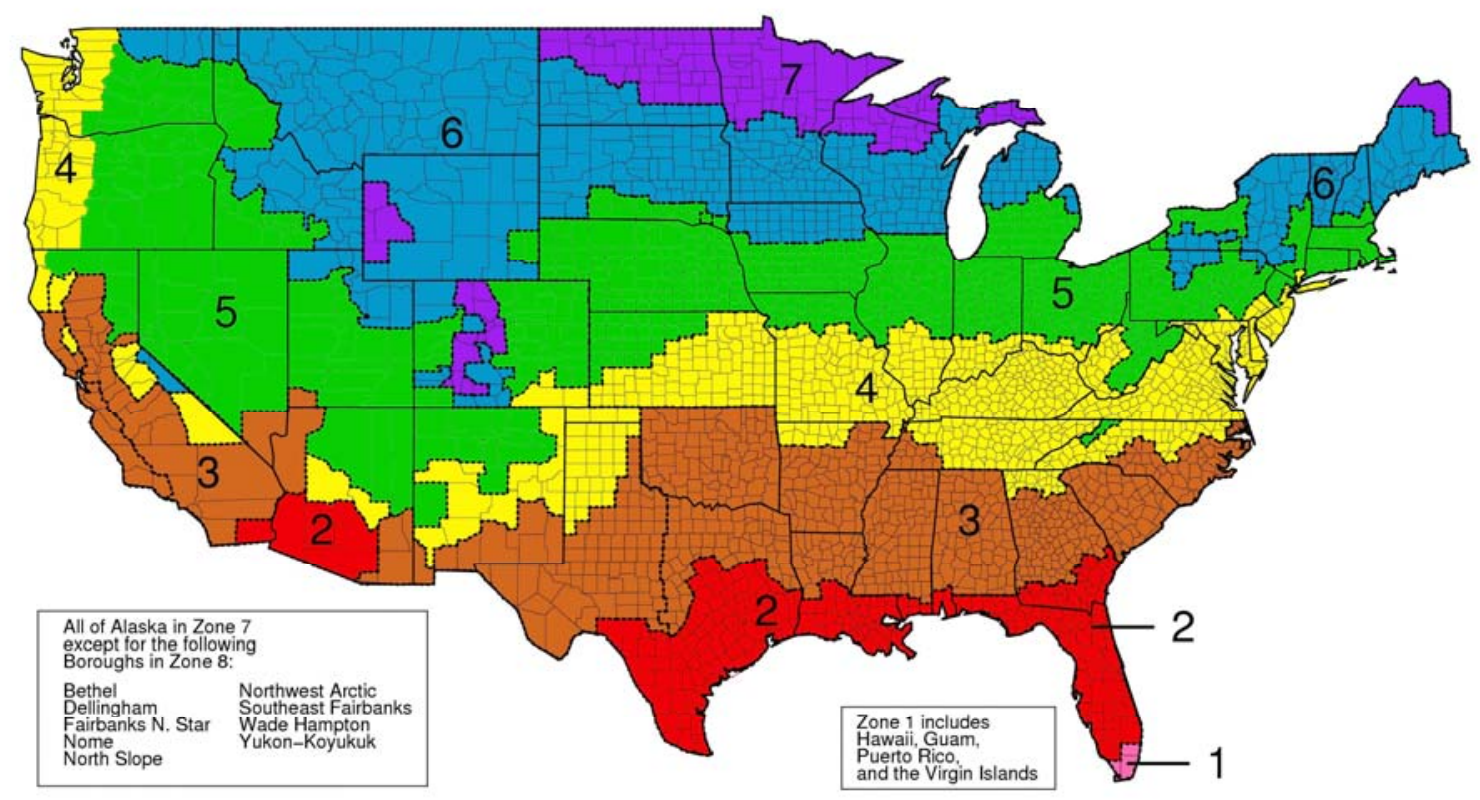

\begin{tabular}{|c|c|c|c|}
\hline \multirow{2}{*}{ Zone } & \multicolumn{2}{|c|}{ Add Insulation to Attic } & \multirow{2}{*}{ Floor } \\
\cline { 2 - 3 } & Uninsulated Attic & Existing 3-4 Inches of Insulation & R13 \\
\hline 1 & R30 to R49 & R25 to R30 & R13 to R19 \\
\hline 2 & R30 to R60 & R25 to R38 & R19 to R25 \\
\hline 4 & R30 to R60 & R25 to R38 & \multirow{2}{*}{ R25 to R30 } \\
\hline 5 to 8 & R38 to R60 & R38 to R49 & R25 to R30 \\
\hline
\end{tabular}

Wall Insulation: Whenever exterior siding is removed on an

Uninsulated wood-frame wall:

- Drill holes in the sheathing and blow insulation into the empty wall cavity before installing the new siding, and

- Zones 3-4: Add R5 insulative wall sheathing beneath the new siding

- Zones 5-8: Add R5 to R6 insulative wall sheathing beneath the new siding.

Insulated wood-frame wall:

- For Zones 4 to 8: Add R5 insulative sheathing before installing the new siding 


\section{How Much Insulation Do I Already Have?}

Look into your attic. We start with the attic because it is usually easy to add insulation to an attic. This table will help you figure out what kind of insulation you have and what its R-value is.

\begin{tabular}{|l|l|l|l|l|}
\hline \multicolumn{2}{|l|}{ What you see: } & $\begin{array}{l}\text { What it } \\
\text { probably is }\end{array}$ & $\begin{array}{l}\text { Depth } \\
\text { (inches) }\end{array}$ & Total R-value \\
\hline $\begin{array}{l}\text { Loose } \\
\text { fibers }\end{array}$ & light-weight yellow, pink, or white & fiberglass & - & $=2.5 \times$ depth \\
\cline { 2 - 5 } & $\begin{array}{l}\text { dense gray or near-white, may } \\
\text { have black specks }\end{array}$ & rock wool & - & $=2.8 \times$ depth \\
\cline { 2 - 5 } & $\begin{array}{l}\text { small gray flat pieces or fibers } \\
\text { (from newsprint) }\end{array}$ & cellulose & - & $=3.7 \times$ depth \\
\hline Granules & light-weight & $\begin{array}{l}\text { vermiculite } \\
\text { or perlite }\end{array}$ & - & $=2.7 \times$ depth \\
\hline Batts & light-weight yellow, pink, or white & fiberglass & & $=3.2 \times$ depth \\
\hline
\end{tabular}

Look into your walls. It is difficult to add insulation to existing walls unless:

- You are planning to add new siding to your house, or

- You plan to finish unfinished space (like a basement or bonus room).

If so, you need to know whether the exterior walls are already insulated or not. One method is to use an electrical outlet on the wall, but first be sure to turn off the power to the outlet. Then remove the cover plate and shine a flashlight into the crack around the outlet box. You should be able to see whether or not insulation is in the wall. Also, you should check separate outlets on the first and second floor, and in old and new parts of the house, because wall insulation in one wall doesn't necessarily mean that it's everywhere in the house. An alternative to checking through electrical outlets is to remove and then replace a small section of the exterior siding.

Look under your floors. Look at the underside of any floor over an unheated space like a garage, basement, or crawlspace. Inspect and measure the thickness of any insulation you find there. It will most likely be a fiberglass batt, so multiply the thickness in inches by 3.2 to find out the R-value (or the R-value might be visible on a product label). If the insulation is a foam board or sprayed-on foam, use any visible label information or multiply the thickness in inches by 5 to estimate the R-value.

Look at your ductwork. Don't overlook another area in your home where energy can be saved - the ductwork of the heating and air-conditioning system. If the ducts of your heating or air-conditioning system run through unheated or uncooled spaces in your home, such as attic or crawlspaces, then the ducts should be insulated. First check the ductwork for air leaks. Repair leaking joints first with mechanical fasteners, then seal any remaining leaks with water-soluble mastic and embedded fiber glass mesh. Never use gray cloth duct tape because it degrades, cracks, and loses its bond with age. If a joint has to be accessible for future maintenance, use pressure- or heat-sensitive aluminum foil tape. Then wrap the ducts with duct wrap insulation of R-6 with a vapor retarder facing on the outer side. All joints where sections of insulation meet should have overlapped facings and be tightly sealed with fiber glass tape; but avoid compressing the insulation, thus reducing its thickness and R-value. 
Return air ducts are often located inside the heated portion of the house where they don't need to be insulated, but they should still be sealed off from air passageways that connect to unheated areas. Drywall- to-ductwork connections should be inspected because they are often poor (or nonexistent) and lead to unwanted air flows through wall cavities. If the return air ducts are located in an unconditioned part of the building, they should be insulated.

Look at your pipes. If water pipes run through unheated or uncooled spaces in your home, such as attic or crawlspaces, then the pipes should be insulated.

\section{Air Sealing}

Air sealing is important, not only because drafts are uncomfortable, but also because air leaks carry both moisture and energy, usually in the direction you don't want. For example, air leaks can carry hot humid outdoor air into your house in the summer, or can carry warm moist air from a bathroom into the attic in the winter.

Most homeowners are aware that air leaks into and out of their houses through small openings around doors and window frames and through fireplaces and chimneys. Air also enters the living space from other unheated parts of the house, such as attics, basements, or crawlspaces. The air travels through:

- $\quad$ any openings or cracks where two walls meet, where the wall meets the ceiling, or near interior door frames;

- gaps around electrical outlets, switch boxes, and recessed fixtures;

- $\quad$ gaps behind recessed cabinets, and furred or false ceilings such as kitchen or bathroom soffits;

- gaps around attic access hatches and pull-down stairs;

- $\quad$ behind bath tubs and shower stall units;

- through floor cavities of finished attics adjacent to unconditioned attic spaces;

- utility chaseways for ducts, etc., and

- plumbing and electrical wiring penetrations.

These leaks between the living space and other parts of the house are often much greater than the obvious leaks around windows and doors. Since many of these leakage paths are driven by the tendency for warm air to rise and cool air to fall, the attic is often the best place to stop them. It's important to stop these leaks before adding attic insulation because the insulation may hide them and make them less accessible. Usually, the attic insulation itself will not stop these leaks and you won't save as much as you expect because of the air flowing through or around the insulation.

There are many fact sheets that will help you stop these air leaks:

- $\quad$ Air sealing

- Air sealing an existing home

- Air Sealing Technology Fact Sheet

- $\quad$ Air Sealing in Occupied Homes (1995) 


\section{Moisture Control and Ventilation}

We talk about moisture control in an insulation fact sheet because wet insulation doesn't work well. Also, insulation is an important part of your building envelope system, and all parts of that system must work together to keep moisture from causing damage to the structure or being health hazards to the occupants. For example, mold and mildew grow in moist areas, causing allergic reactions and damaging buildings.

When Is Moisture a Problem?

When moist air touches a cold surface, some of the moisture may leave the air and condense, or become liquid. If moisture condenses inside a wall, or in your attic, you will not be able to see the water, but it can cause a number of problems. Adding insulation can either cause or cure a moisture problem. When you insulate a wall, you change the temperature inside the wall. That can mean that a surface inside the wall, such as the sheathing behind your siding, will be much colder in the winter than it was before you insulated. This cold surface could become a place where water vapor traveling through the wall condenses and leads to trouble. The same thing can happen within your attic or under your house. On the other hand, the new temperature profile could prevent condensation and help keep your walls or attic drier than they would have been.

\section{Four Things You Can Do to Avoid Moisture Problems:}

1. Control liquid water. Rain coming through a wall, especially a basement or crawlspace wall, may be less apparent than a roof leak, especially if it is a relatively small leak and the water remains inside the wall cavity. Stop all rain-water paths into your home by:

- making sure your roof is in good condition,

- caulking around all your windows and doors, and

- $\quad$ keeping your gutters clean - and be sure the gutter drainage flows away from your house.

- If you replace your gutters, choose larger gutters and gutter guards to help keep rain from dripping onto the ground near the house.

Be sure that the condensate from your air conditioner is properly drained away from your house. You should also be careful that watering systems for your lawn or flower beds do not spray water on the side of your house or saturate the ground near the house. It is also a good idea to check the caulking around your tub or shower to make sure that water is not leaking into your walls or floors. You can place thick plastic sheets on the floor of your crawlspace to keep any moisture in the ground from getting into the crawlspace air, and then into your house.

2. Ventilate. You need to ventilate your home because you and your family generate moisture when you cook, shower, do laundry, and even when you breathe. More than $99 \%$ of the water used to water plants eventually enters the air. If you use an unvented natural gas, propane, or kerosene space heater, all the products of combustion, including water vapor, are exhausted directly into your living space. 
This water vapor can add 5 to 15 gallons of water per day to the air inside your home. If your clothes dryer is not vented to the outside, or if the outdoor vent is closed off or clogged, all that moisture will enter your living space. Just by breathing and perspiring, a typical family adds about 3 gallons of water per day to their indoor air. You especially need to vent your kitchen and bathrooms. Be sure that these vents go directly outside, and not to your attic, where the moisture can cause problems. Remember that a vent does not work unless you turn it on; so if you have a vent you are not using because it is too noisy, replace it with a quieter model. If your attic is ventilated, it is important that you never cover or block attic vents with insulation. Take care to prevent loose-fill insulation from clogging attic vents by using baffles or rafter vents. When you think about venting to remove moisture, you should also think about where the replacement air will come from, and how it will get into your house. When natural ventilation has been sharply reduced with extra air-sealing efforts, it may be necessary to provide fresh air ventilation to avoid build-up of stale air and indoor air pollutants. Special air-to-air heat exchangers, or heat- recovery ventilators, are available for this purpose. For more information about controlled ventilation, see the Whole-House Ventilation Systems Technology Fact Sheet.

3. Stop Air Leaks. It is very important to seal up all air-leakage paths between your living spaces and other parts of your building structure. Measurements have shown that air leaking into walls and attics carries significant amounts of moisture. Remember that if any air is leaking through electrical outlets or around plumbing connections into your wall cavities, moisture is carried along the same path. The same holds true for air moving through any leaks between your home and the attic, crawlspace, or garage. Even very small leaks in duct work can carry large amounts of moisture, because the airflow in your ducts is much greater than other airflows in your home. This is especially a problem if your ducts travel through a crawlspace or attic, so be sure to seal these ducts properly (and keep them sealed!). Return ducts are even more likely to be leaky, because they often involve joints between drywall and ductwork that may be poorly sealed, or even not sealed at all.

4. Plan a moisture escape path. Typical attic ventilation arrangements are one example of a planned escape path for moisture that has traveled from your home's interior into the attic space. Cold air almost always contains less water than hot air, so diffusion usually carries moisture from a warm place to a cold place. You can let moisture escape from a wall cavity to the dry outdoors during the winter, or to the dry indoors during the summer, by avoiding the use of vinyl wall coverings or low-perm paint. You can also use a dehumidifier to reduce moisture levels in your home, but it will increase your energy use and you must be sure to keep it clean to avoid mold growth. If you use a humidifier for comfort during the winter months, be sure that there are no closed-off rooms where the humidity level is too high. 


\section{INSULATION INSTALLATION PRECAUTIONS}

-Wear clothing adequate to protect against skin contact and irritation. A long-sleeved shirt with collar and cuffs buttoned, gloves, hat, glasses, and disposable dust respirator are advisable in all do-it-yourself insulation projects. Also, read the label and follow all the manufacturer's directions.

- Do not cover or hand-pack insulation around bare stove pipes, electrical fixtures, motors, or any heat-producing equipment such as recessed lighting fixtures. If you pack insulation around these heat-producing locations, the heat can build up, leading to fire. Electrical fire-safety codes prohibit the installation of thermal insulation within three inches of a recessed fixture enclosure, wiring compartment, or ballast, or above the fixture so that it will trap heat and prevent free circulation of air, unless the fixture is identified by label as suitable for insulation to be in direct contact with the fixture. THIS IS FOR FIRE SAFETY.

- Do not cover attic vents with insulation. Proper ventilation must be maintained to avoid overheating in summer and moisture build-up all year long.

Whether you install the insulation yourself or have it done by a contractor, it is a good idea to educate yourself about proper installation methods because an improper installation can reduce your energy savings.

Also, if your house is very old, you may want to have an electrician check to see if:

- the electrical insulation on your wiring is degraded,

- the wires are overloaded, or

- $\quad$ knob and tube wiring was used (often found in homes built before 1940).

If any of these wiring situations exists in your house, it may be hazardous to add thermal insulation within a closed cavity around the wires because that could cause the wires to overheat. THIS IS FOR FIRE SAFETY. The National Electric Code forbids the installation of loose, rolled, or foam-in-place insulation around knob and tube wiring.

\section{IF YOU HAVE IT DONE PROFESSIONALLY}

You should obtain cost estimates from several contractors for a stated R-value. Make sure you describe the job in writing in the same terms to each one. You may want to ask each contractor about their air- sealing services as well. Remember that you want good quality materials and labor, as well as a low price. Do not be surprised to find the quoted prices for a given R-value installation to vary by a factor of two or more. When you talk to a contractor, talk of R-values. Don't forget that R-values are determined by material type, thickness, and installed weight per square foot, not by thickness alone.

Adding thermal insulation to the ceiling or walls of a mobile home is complex and usually requires installation by specialists.

If adding insulation over existing insulation, do NOT use a vapor barrier between the two layers! 


\section{$\underline{\text { Attics }}$}

On unfinished attic floors, work from the perimeter toward the attic door. Be careful about where you step in the attic. Walk only on the joists so that you won't fall through the drywall ceiling. You may need to place walking boards across the tops of the joists to make the job easier. Remember that it is important to seal up air leaks between your living space and the attic before adding insulation in your attic.

Installing batts and rolls in attics is fairly easy, but doing it right is very important. Use unfaced batts, especially if reinsulating over existing insulation. If there is not any insulation in your attic, fit the insulation between the joists. If the existing insulation is near or above the top of the joists, it is a good idea to place the new batts perpendicular to the old ones because that will help to cover the tops of the joists themselves and reduce thermal bridging through the frame. Also, be sure to insulate the trap or access door. Although the area of the door is small, an uninsulated attic door will reduce energy savings substantially.

For blown-in loose fill insulation, each bag of insulating material used by the contractor should be marked with an R-value for the area to be covered. Although these figures may differ among manufacturers, the area figure will tell you the right number of bags to be used. Similarly, packages of other types of insulation should be identified by their R-value. It is important that you check that the proper amount is installed in your residence. Ask the contractor to attach vertical rulers to the joists prior to a loose-fill installation in your attic to help you see that the proper depth was installed. Also, the installer must provide a signed and dated statement describing the insulation installed, stating thickness, coverage area, R- value, and number of bags installed. In some areas, infrared thermography services are offered to help discover any gaps in the insulation.
In some houses, it is easier to get complete coverage of the attic floor with blown-in loose-fill insulation. It is best to hire an insulation contractor for this job. Loose-fill insulation must be prevented from shifting into vents or from contacting heatproducing equipment (such as recessed lighting fixtures). Block off those areas with baffles or retainers to hold the loose-fill insulation in place.

When you stack new insulation on top of existing attic insulation, the existing insulation is compressed a small amount. This will slightly decrease the R-value of the existing insulation. This effect is most important if the new insulation is more dense than the old insulation. You can compensate for this stacking effect and achieve the desired total R-value by adding about one extra inch of insulation if the old insulation is fiber glass, or about $1 / 2$ inch if the old insulation is rock wool or cellulose.

Reflective Systems are installed in a manner similar to placing batts and blankets. Proper installation is very important if the insulation is to be effective. Study and follow the manufacturer's instructions. Often, reflective insulation materials have flanges that are to be stapled to joists. Since reflective foil will conduct electricity, avoid making contact with any bare electrical wiring.

Radiant barriers may be installed in attics in several configurations. The radiant barrier is most often attached near the roof, to the bottom surface of the attic truss chords or to the rafter framing. Do not lay a radiant barrier on top of your insulation or on the attic floor because it will soon be covered with dust and will not work. A separate DOE fact sheet is available for radiant barriers to show which parts of the country are most likely to benefit from this type of system. 
If your attic has NO insulation, you may decide to insulate the underside of the roof instead of the attic floor. (This option is more often used in new houses and is described in Design Option: ATTIC VENTILATION OR A CATHEDRALIZED ATTIC). If you choose the cathedralized attic approach, all attic vents must be sealed. Spray-foam is then often used to insulate the underside of the roof sheathing. If batts are used for this purpose, they must be secured in a manner similar to that described below for insulating under floors. It is best to hire an insulation contractor with experience in this type of installation for this job.

$\underline{\text { Walls }}$

Installing insulation in the cavity of exterior walls is difficult. However, when new siding is to be installed, it is a good idea to consider adding thermal insulation under the new siding. The Retrofit Best Practices Guide provides useful information about adding insulation when you remodel the outside of your house. It usually requires the services of a contractor who has special equipment for blowing loose-fill insulation into the cavity through small holes cut through the sidewall, which later are closed.

It is sometimes feasible to install rigid insulation on the outdoor side of masonry sidewalls such as concrete block or poured concrete. However, if that is not an option, you can use rigid insulation boards or batts to insulate the interior of masonry walls. To install boards, wood furring strips should be fastened to the wall first. These strips provide a nailing base for attaching interior finishes over the insulation. Fire safety codes require that a gypsum board finish, at least $1 / 2$ inch thick, be placed over plastic foam insulation. The gypsum board must be attached to the wood furring strips or underlying masonry using nails or screws.

The first-floor band joist may be accessible from the basement or crawlspace. Make sure it is properly insulated as shown in Figure 1. More detailed drawings and insulation techniques for the band joist are shown in the Wall Insulation Technology Fact Sheet.

\section{Basement Walls}

When using batt or rigid insulation to insulate the inside of concrete basement walls, it is necessary to attach wood furring strips to the walls by nailing or bonding, or to build an interior stud-wall assembly on which the interior finish can be attached after the insulation is installed. The cavity created by the added framing should be thick enough for the desired insulation R-value. 
The kraft paper or standard foil vapor retarder facings on many blanket insulation products must be covered with gypsum or interior paneling because of fire considerations. Some blanket products are available without these facings or with a special flame resistant facing (labeled FS25 - or flame spread index 25) for places where the facing would not be covered. Sometimes the flame-resistant cover can be purchased separately from the insulation. Also, there are special fiber glass blanket products available for basement walls that require less framing and can be left exposed. These blankets have a flame-resistant facing and are labeled to show that they comply with ASTM C 665, Type II, Class A.

More information is given in the Basement Insulation Technology Fact Sheet.

\section{$\underline{\text { Floors and Crawlspaces }}$}

If you have a floor over a crawlspace, you can EITHER:

- Insulate the underside of the floor and ventilate the crawlspace, OR

- Leave the floor uninsulated and insulate the walls of an unventilated crawlspace.

When batts or rolls are used on the underside of a floor above an unheated crawlspace or basement, fit the insulation between the beams or joists and push it up against the floor overhead as securely as possible without excessive compaction of the insulation. The insulation can be held in place, either by tacking chicken wire (poultry netting) to the edges of the joist, or with snap-in wire holders. Batts and rolls must be cut and fit around cross-bracing between floor joists or any other obstructions. Strips of insulation may be cut off and stuffed into tight spaces by hand. Don't forget to place insulation against the perimeter that rests on the sill plate. If you insulate above an unheated crawlspace or basement, you will also need to insulate any ducts or pipes running through this space. Otherwise, pipes could freeze and burst during cold weather.

Reflective Systems are installed in a manner similar to placing batts. Proper installation is very important if the insulation is to be effective. Study and follow the manufacturer's instructions. Often, reflective insulation materials have flanges that are to be stapled to floor joists. Since reflective foil will conduct electricity, one must avoid making contact with any bare electrical wiring.

Spray-foam can be used to insulate the underside of a floor. The spray foam can do a good job of filling in the space around wires and other obstructions and in filling any oddly-shaped areas. It is best to hire an insulation contractor with experience in this type of installation.

When a fiberglass blanket is used to insulate the walls of an unventilated crawlspace, it is sometimes necessary to attach wood furring strips to the walls by nailing or bonding. The insulation can then be stapled or tacked into place. Alternatively, the insulation can be fastened to the sill plate and draped down the wall. You should continue the insulation over the floor of the crawlspace for about two feet on top of the required ground vapor retarder. Because the insulation will be exposed, be sure to use either an unfaced product or one with the appropriate flame spread rating. When rigid foam insulation boards are used to insulate the walls of an unventilated crawlspace, they can be bonded to the wall using recommended adhesives. Because the insulation will be exposed, be sure to check the local fire codes and the flame-spread rating of the insulation product. If you live in an area prone to termite damage, check with a pest control professional to see if you need to provide for termite inspections. 


\section{Resources and Links}

Additional and more detailed information about thermal insulation materials and installation and about energy conservation in buildings is available from the agencies and organizations listed below. Your public utility company may also provide information and assistance on home energy conservation practices and materials.

\section{Internet Sites}

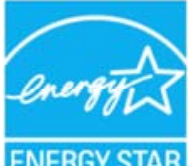

Energy Star Programs supported by the Environmental Protection Agency and the Department of Energy. These programs include information about Energy Star new

ENERGY STAR homes and home improvement, http://www.energystar.gov

Building Envelope Research topics, see http://www.ornl.gov/roofs+walls

Building Technology Program Publications, see "Technology Fact Sheets"

http://www.eere.energy.gov/buildings/info/publications.html\#technology\%20fact\%20sheets

\section{Computer Programs}

ZIP-Code Computer Program:

Operable version found on the internet at: http://www.ornl.gov/ roofs/Zip/ZipHome.html

Home Energy Saver, a do-it-yourself energy audit tool produced by Lawrence Berkeley National Laboratory's Center for Building Science, http://homeenergysaver.lbl.gov

\section{Reference Sources}

U.S. Department of Energy, Energy Efficiency and Renewable Energy Program Office Home Page, http://www.eere.energy.gov

A Consumer's Guide to Energy Efficiency and Renewable Energy in the Home http://www.eere.energy.gov/consumer/your_home/

Tips for Energy Savers http://www1.eere.energy.gov/consumer/tips/

Insulation Contractors Association of America (ICAA) Phone 703-739-0356

http://www.insulate.org/

National Association of Home Builders (NAHB)

$120115^{\text {th }}$ Street NW

Washington, DC 20005

Phone: 202-266-8200 x0, 800-368-5242, Fax: 202-266-8400

http://www.nahb.org/ 
NAHB Research Center, Inc.

400 Prince Georges Boulevard

Upper Marlboro, MD 20774

Phone: 800-638-8556

http://www.nahbrc.com

National Institute of Building Sciences

1090 Vermont Avenue, NW, Suite 700

Washington, DC 20005-4905

Phone: 202-289-7800, Fax: 202-289-1092

http://www.nibs.org/

\section{Other Publications}

\section{U.S. Department of Energy}

Energy Efficiency and Renewable Energy Information Center

http://www1.eere.energy.gov/informationcenter/

Office of Scientific and Technical Information (OSTI)

P.O. Box 62

Oak Ridge, TN 37831

Phone: 865-576-1188, Fax: 865-576-2865 http://www.osti.gov/

- $\quad$ Retrofit Best Practices Guide,ORNL/TM-2003/286

- Radiant Barrier Fact Sheet, DOE/CE-0335P

- Builder's Foundation Handbook, ORNL/CON-295

- Moisture Control Handbook, ORNL/SUB--89--SD350/1

- $\quad$ Supporting Documentation for the 1997 Revision to the DOE Insulation Fact Sheet, ORNL6907

U.S. Department of Housing and Urban Development

Washington, DC 20410-6000 Energy Efficient Rehab Advisor

\section{Guidelines for New Home Construction}

Building Standards and Guidelines Program (BSGP) http://www.energycodes.gov

Other Building Code Links http://www.energycodes.gov/links.stm\#code

ASHRAE STANDARD 90.2-2007 Energy-Efficient Design of New Low-Rise Residential Buildings

American Society of Heating, Refrigerating and Air-Conditioning Engineers, Inc.

1791 Tullie Circle, NE

Atlanta, GA 30329 http://www.ashrae.org

Thermal Design Guide for Exterior Walls

American Iron and Steel Institute

http://www.toolbase.org/PDF/DesignGuides/ThermalDesignGuide.pdf 


\section{Manufacturer's Associations}

Air Diffusion Council (ADC) (representing manufacturers of flexible air duct) 1901 N. Roselle Road, Suite 800

Schaumburg, Illinois 60195

Phone: 847-706-6750, Fax: 847-706-6751

http://www.flexibleduct.org/

American Iron and Steel Institute

1140 Connecticut Ave., NW, Suite 705

Washington, D.C. 20036

Phone: 202-452-7100

http://www.steel.org/

Cellulose Insulation Manufacturers Association (CIMA)

136 South Keowee Street

Dayton, $\mathrm{OH} 45402$

Phone: 888-881-CIMA (881-2462) or 937-222-CIMA (222-2462), Fax:937-222-5794

http://www.cellulose.org/

Center for the Polyurethanes Industry

American Chemistry Council

1300 Wilson Blvd., Arlington, VA 22209

http://www.polyurethane.org

Expanded Polystyrene (EPS) Molder's Association

1298 Cronson Boulevard, Suite 201

Crofton, MD 21114

Phone: 800-607-3772

http://www.epsmolders.org

Extruded Polystyrene Foam Association (XPSA)

4223 Dale Boulevard

Woodbridge, VA 22193

Phone: 703-730-0062, Fax: 703-583-5860

http://www.xpsa.com

Home Ventilating Institute

1000 N. Rand Rd., Suite 214

Wauconda, IL 60084

Phone: 847-526-2010, Fax: 847-526-3993

http://www.hvi.org/ 
Insulating Concrete Form Association

1730 Dewes Street, Suite \#2

Glenview, IL 60025

Phone: 888-864-4232, Fax: 847-657-9728

http://www.forms.org/

North American Insulation Manufacturers Association (NAIMA)

44 Canal Center Plaza, Suite 310

Alexandria, VA 22314

Phone: 703-684-0084, Fax: 703-684-0427

http://www.naima.org

Polyisocyanurate Insulation Manufacturers Association (PIMA)

7315 Wisconsin Avenue, Suite 400E

Washington, DC 20814

Phone: 301-654-0000, Fax: 301-951-8401

http://www.pima.org

Reflective Insulation Manufacturers Association

4519 E. Lone Cactus Drive

Phoenix, AZ 85050

Phone 800-279-4123, Fax: 480-513-4749

http://www.rima.net/

Society of the Plastics Industry, Inc.

1667 K St., NW, Suite 1000

Washington, DC 20006

Phone: 202-974-5200, Fax: 202-296-7005

http://www.socplas.org

Spray Polyurethane Foam Alliance

4400 Fair Lakes Court, Suite 105

Fairfax, VA 22033

Phone: 800-523-5154, Fax: 703-222-5816

http://www.sprayfoam.com/

Structural Insulated Panel Association (SIPA)

P.O. Box 1699

Gig Harbor, WA 98335

Phone: 253-858-7472, Fax: 253-858-0272

http://www.sips.org 


\title{
About This Fact Sheet
}

The DOE Insulation Fact Sheet offers helpful advice on insulating your existing house and supplies information you need to know if you are building a new home. It is available in two versions - portable document format (pdf) and on-line version you are reading here. In order to view the pdf version you must have Adobe Acrobat Reader installed on you computer. The web-based version includes recommended insulation levels based on the ZIP code of a locale.

\section{PDF Version}

HTML Version

The insulation levels recommended in this Fact Sheet were chosen based on a life-cycle cost analysis. This analysis includes many assumptions about system efficiencies, and what rate of return you would like to earn on your investment. If you want to find out more about how the recommended insulation levels were chosen, please see the Supporting Documentation.

2008 Addendum to Supporting Documentation 2002 Addendum to Supporting Documentation

Supporting Documentation

\author{
Prepared by \\ Oak Ridge National Laboratory \\ Oak Ridge, Tennessee 37831-6283 \\ managed by \\ UT-Battelle LLC \\ for the \\ U.S. DEPARTMENT OF ENERGY \\ under contract DE-AC05-00OR22725 \\ for \\ The Building Technology Program \\ Office of Energy Efficiency and Renewable Energy \\ 1000 Independence Avenue, SW \\ Washington, DC 20585-0121 \\ Building Envelope Research \\ Oak Ridge National Laboratory
}

For more information, contact the program manager for Building Envelope Research:

André O. Desjarlais

Oak Ridge National Laboratory

P. O. Box 2008, MS 6070

Oak Ridge, TN 37831-6070

E-mail Andre Desjarlais 